\title{
The Role of Structure Evolution for PrBaCo206- $\delta$ in Oxygen Reduction Reaction Kinetics at SOFC Cathode
}

\author{
Nan Zhang \\ nnzhang@mix.wvu.edu
}

Follow this and additional works at: https://researchrepository.wvu.edu/etd

Part of the Engineering Science and Materials Commons

\section{Recommended Citation}

Zhang, Nan, "The Role of Structure Evolution for PrBaCo206-ס in Oxygen Reduction Reaction Kinetics at SOFC Cathode" (2021). Graduate Theses, Dissertations, and Problem Reports. 8072.

https://researchrepository.wvu.edu/etd/8072

This Dissertation is protected by copyright and/or related rights. It has been brought to you by the The Research Repository @ WVU with permission from the rights-holder(s). You are free to use this Dissertation in any way that is permitted by the copyright and related rights legislation that applies to your use. For other uses you must obtain permission from the rights-holder(s) directly, unless additional rights are indicated by a Creative Commons license in the record and/ or on the work itself. This Dissertation has been accepted for inclusion in WVU Graduate Theses, Dissertations, and Problem Reports collection by an authorized administrator of The Research Repository @ WVU.

For more information, please contact researchrepository@mail.wvu.edu. 


\title{
The Role of Structure Evolution for $\mathrm{PrBaCo}_{2} \mathrm{O}_{6-\delta}$ in Oxygen Reduction Reaction Kinetics at SOFC Cathode
}

\author{
Nan Zhang \\ Dissertation submitted \\ to the Benjamin M. Statler College of Engineering and Mineral Resources \\ at West Virginia University \\ in partial fulfillment of the requirements for the degree of \\ Doctor of Philosophy in \\ Mechanical and Aerospace Engineering \\ Xingbo Liu, Ph.D., Chair \\ Harry O. Finklea, Ph.D. \\ Edward M. Sabolsky, Ph.D. \\ David Mebane, Ph.D. \\ Konstantinos A Sierros, Ph.D. \\ Daniel J. Haynes, Ph.D. \\ Department of Mechanical and Aerospace Engineering \\ Morgantown, West Virginia \\ 2021
}

Keywords: Solid Oxide Fuel Cells; PrBaCo2O6- $;$; Tetragonal; Orthorhombic Copyright 2021 Nan Zhang 


\begin{abstract}
The Role of Structure Evolution for $\mathrm{PrBaCo}_{2} \mathrm{O}_{6-8}$ in Oxygen Reduction Reaction Kinetics at SOFC Cathode

Nan Zhang
\end{abstract}

Mixed ion and electron conductive materials especially those with perovskite-related structures have been widely used as cathode and anode of solid oxide fuel cells (SOFC). Double perovskite $\mathrm{LnBaCo}_{2} \mathrm{O}_{6-\delta}(\mathrm{Ln}=\mathrm{Pr}, \mathrm{Nd}, \mathrm{Sm}, \mathrm{Nd}, \mathrm{Y})$ cathodes have been shown better performance than single perovskite $\mathrm{Ln}_{0.5} \mathrm{Ba}_{0.5} \mathrm{CoO}_{3-\delta}$ in $\mathrm{SOFC}$ cathode studies. However, the effect of different double perovskite phases on oxygen transport properties has not been investigated. In this study, the electrochemical performance of different double perovskite materials $\mathrm{PrBaCo}_{2} \mathrm{O}_{6-\delta}(\mathrm{PBCO})$, $\mathrm{NdBaCo}_{2} \mathrm{O}_{6-\delta}(\mathrm{NBCO}), \mathrm{YBaCo}_{2} \mathrm{O}_{6-\delta}(\mathrm{YBCO})$, and single perovskite $\mathrm{La}_{0.5} \mathrm{Ba}_{0.5} \mathrm{CoO}_{3-\delta}$ (LBCO) were investigated and compared. The PBCO material was determined to be a potential SOFC cathode with excellent electrocatalytic activity to oxygen reduction. Tetragonal and orthorhombic PBCO perovskite powders are synthesized by controlling the sintering atmosphere and temperatures to study and compare the oxygen transport mechanism. In-situ X-ray Diffraction (XRD) was adopted to characterize the structure of PBCO in the SOFC cathode operation range, and it proves that there is a phase transition that occurred for pristine orthorhombic $\mathrm{PBCO}$ when above $550{ }^{\circ} \mathrm{C}$. The oxygen vacancy in tetragonal $\mathrm{PBCO}$ was proved to be disordered by the Neutron Powder Diffraction (NPD) characterization, while that in orthorhombic was ordered distributed. The Selected Area Electron Diffraction (SAED) patterns and the High-Resolution Transmission Electron Microscopy (HRTEM) morphology also tells that PBCO treated in the air has a higher disorder degree than that of treated in nitrogen. The 
oxygen non-stoichiometry $\delta$, a critical factor to electro-catalytic activity on oxygen reduction, was determined by Thermal Gravimetric Analysis (TGA). Further, thermodynamic quantities were obtained through the solving of the thermodynamic equation. The abnormal partial molar entropy and enthalpy values were related to the complex activation of oxygen vacancy caused by the phase transition, the appearance of a new crystal lattice that has been detected by NPD. The oxygen transport on dense PBCO electrodes has been investigated with the Electrical Conductivity Relaxation (ECR) technique. The study of oxygen bulk diffusion coefficients together with the structure factor from NPD and the thermodynamic property from TGA have promoted the possibility of calculating the migration entropy and enthalpy. The performance of PBCO oxides as SOFC cathodes was evaluated by electrochemical impedance spectroscopy. Both the as-synthesized tetragonal and orthorhombic show good electrocatalytic activity to oxygen reduction, with polarization resistance $0.078 \Omega \cdot \mathrm{cm}^{2}$ and $0.10 \Omega \cdot \mathrm{cm}^{2}$ respectively at 650 ${ }^{\circ} \mathrm{C}$. Oxygen bulk diffusion and surface chemical exchange co-limited the oxygen reduction reaction (ORR) on the $\mathrm{PBCO}$ cathodes. Besides, oxygen reduction reactions on electrodes treated in nitrogen were retard mainly by oxygen incorporation into the lattice step. 


\section{Acknowledgments}

I had received plenty of support and help with the dissertation from many wonderful people.

My deepest gratitude goes first and foremost to my advisor Dr. Xingbo Liu for his guidance, patience, and expertise. In all these years, Dr. Liu uses his way to foster my confidence, train me to become a critical thinker, and boost my research skills. Dr. Liu has always used his acuity to polish my research ideas and has my head screwed on the right track. Many times, he put my interests in the first place and prioritized my request so that I could finish the program. I am also extremely grateful to Dr. Daniel Haynes, Dr. Harry Finklea, Dr. Edward Sabolsky, Dr. David Mebane, and Dr. Konstantinos Sierros for their constructive suggestions on my work, as well as being my committee members.

Besides, I will also thank several people who give me significant help with my research. Dr. Yang Yu helped me with TEM characterization. Dr. Simon Kimber helped me with the NPD test and analysis. Dr. William Benincosa helped with the TG test.

I would like to express my gratitude to Dr. Dushyant Shekhawat, Dr. David Berry, Wayne Surdoval, who always support and encourage me to try something novel and bold in my research project at NETL. I would like to thank the faculty member Dr. Wenyuan Li for the many discussions on the thesis, Dr. Victor Mucino, and Sarah Lowery for the administrative support on my research progress.

Also, I would like to thank my group members, Bo Guan, Xiujuan Chen, Gregory Collins, Shanshan Hu, Zhipeng Zeng, Yi Wang, He Qi, et al. Their extreme kindness, friendliness, and support help me overcome all difficulties and achieve each milestone in these years. These friends will be a priceless fortune in my whole life. In addition, my great appreciation also goes to Dr. Lixun Su, Cindy Liu, who encouraged me to complete the Ph.D. program and to pursue an academic career in the U.S.

Finally, I would like to thank my parents for their unconditional love and support. It is not easy for them to decide to send their child to the U.S. for a doctoral program, but they always support and feel proud of all my decisions. 


\section{Table of Contents}

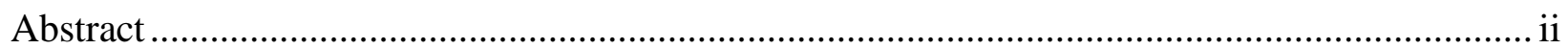

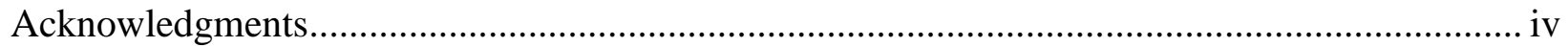

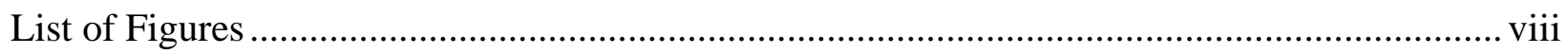

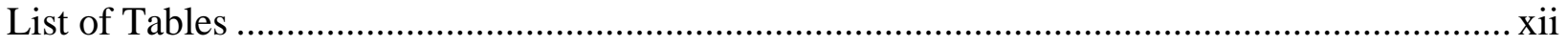

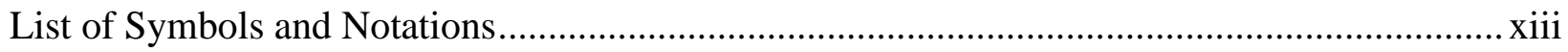

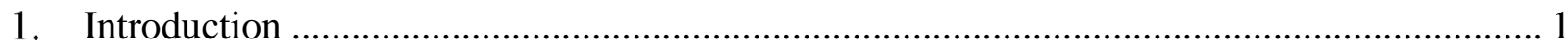

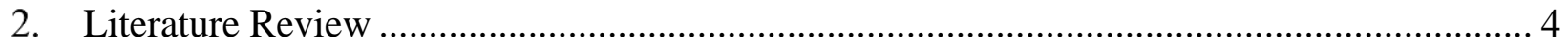

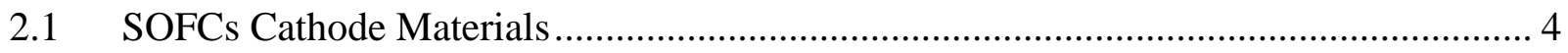

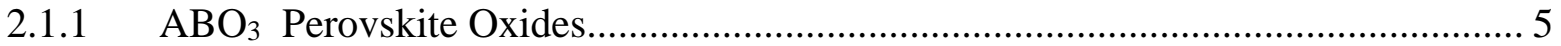

2.1.2 Ordered Double-Layer Perovskite Oxides ............................................................ 7

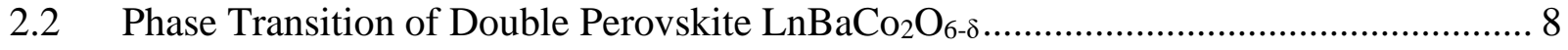

2.3 Anisotropic Oxygen Ion Diffusion in Layered Cathodes............................................... 11

2.4 Oxygen Reduction in the Surface Process Limited Cathode …………………............. 15

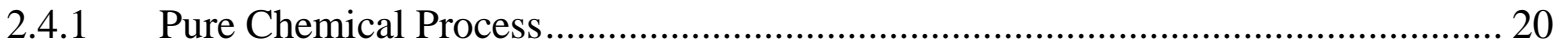

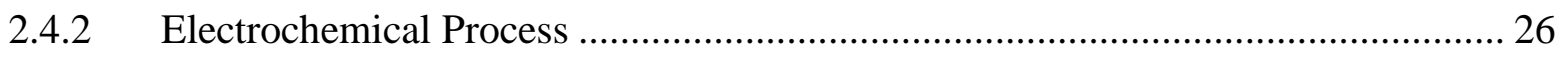

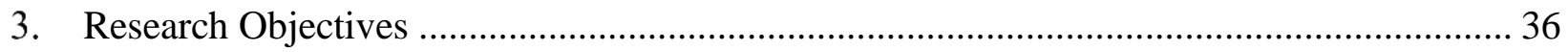

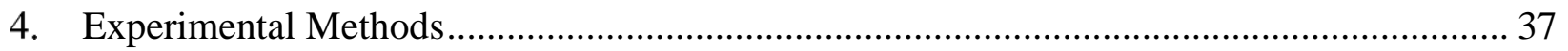

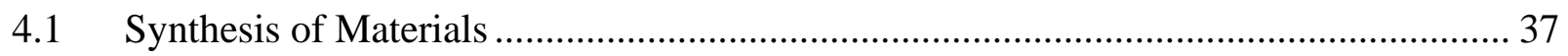

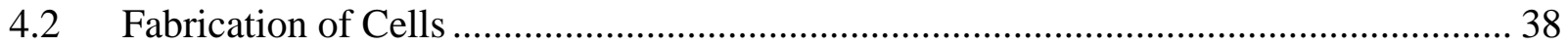

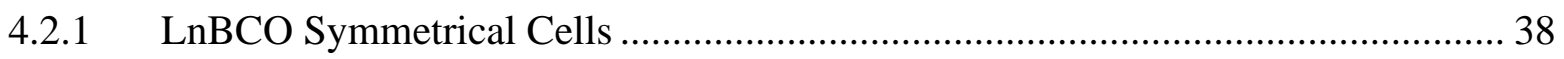

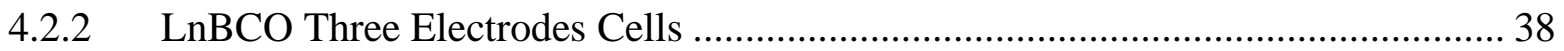

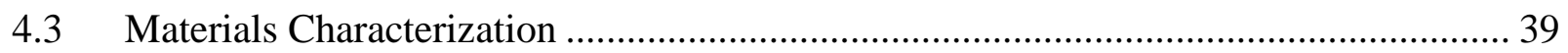


4.3.1 XRD, SEM, and EDS....... 39

4.3.2 Transmission Electron Microscopy Sample Preparation and Characterization...... 40

4.3.3 Neutron Powder Diffraction and Rietveld Refinement at Oak Ridge National

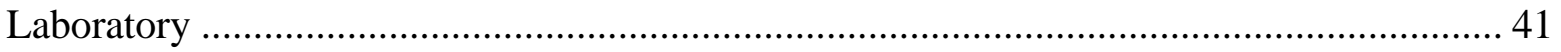

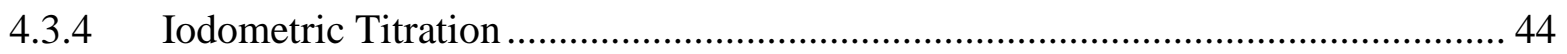

4.3.5 Thermal Gravimetric Analysis (TGA)/Differential Thermal Analysis (DTA)...... 44

4.3.6 BET Surface Area ..................................................................................... 45

4.3.7 Temperature Programmed Oxidation-TPO and $\mathrm{O}_{2}$ Pulse Chemisorption ............. 45

4.3.8 Temperature Programmed Desorption.................................................... 47

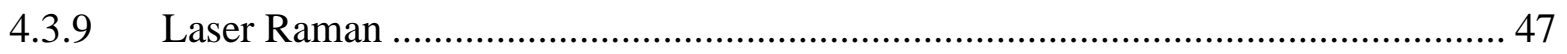

4.4 Electrical and Electrochemical Characterization .............................................. 48

4.4.1 Four-point DC Method Conductivity Measurement.......................................... 48

4.4.2 Electrical Conductivity Relaxation (ECR) ............................................. 49

4.4.3 Electrochemical Impedance Spectroscopy (EIS) ...................................... 51

5. Materials Structure and Performance of LnBCO-Based Cathode..................................... 53

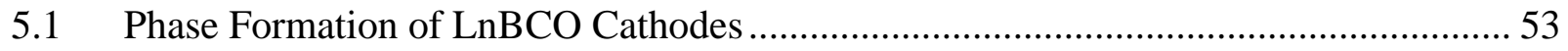

5.2 Electrical Conductivity of LnBCO Cathodes ................................................. 56

5.3 Oxygen Reduction Reaction Performance of LnBCO cathodes ..............................59

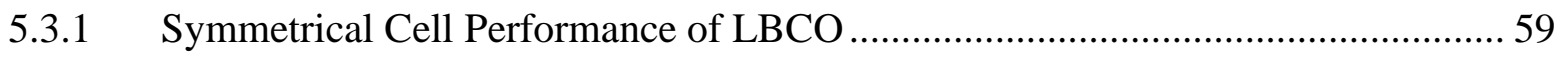

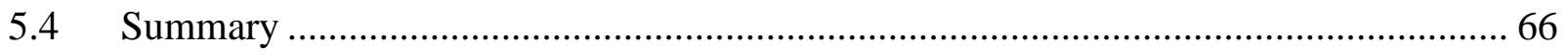

6. The structure Evolution of $\mathrm{PrBaCo}_{2} \mathrm{O}_{6-\delta}$ and the Influence on the Thermodynamic Property 68

6.1 The Lattice Structure of PBCO and Refinement.................................................. 68 
6.2 Lattice Oxygen Vacancy Order/Disorder Transition ........................................... 70

6.3 Electron Microscopy Investigations of PBCO ......................................................... 72

6.4 Oxygen Non-stoichiometry Determined by TGA ............................................... 79

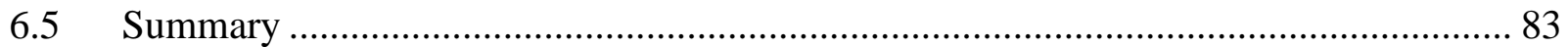

7. Oxygen Transport Mechanism of Dense PBCO and ORR Analysis on Porous PBCO

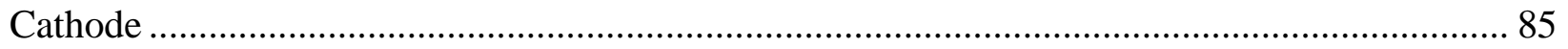

7.1 $\mathrm{K}$ and D Calculated from the Fitting of the ECR Curve ........................................... 86

7.2 Oxygen Bulk Diffusion Comparison Between Tetragonal and Orthorhombic PBCO .. 90

7.3 Factors Determining Catalytic Activity in $\mathrm{O}_{2}$ Reduction on $\mathrm{PBCO}$.......................... 95

7.3.1 Symmetrical Cell Performance ............................................................... 96

7.4 Rate Limiting Steps on PBCO Cathodes ........................................................ 100

7.4.1 Oxygen Reaction Order and Rate-determining Steps Analysis ......................... 100

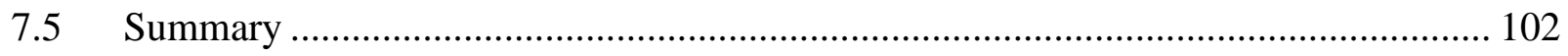

8. Conclusions and Recommendation Work .............................................................. 104

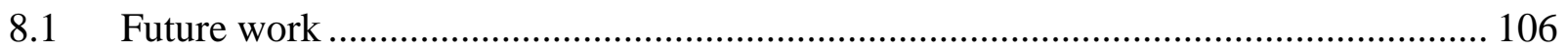

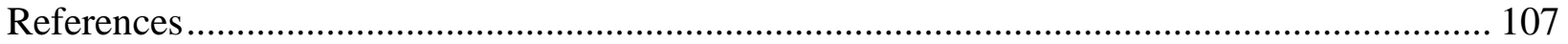




\section{List of Figures}

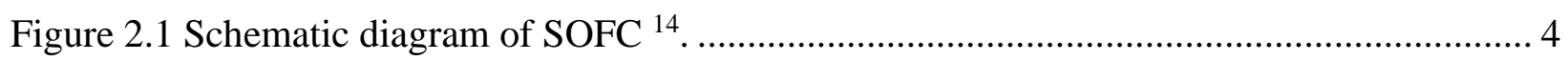

Figure 2.2 Schematic diagram of the $\mathrm{ABO}_{3}$ perovskite structure.............................................. 6

Figure 2.3 Schemes perovskite LBCO: (a) cubic, (b) orthorhombic, and (c) tetragonal ${ }^{53,54} \ldots \ldots \ldots .8$

Figure 2.4 Arrhenius plot of (a) the oxygen tracer diffusion and (b) the surface exchange coefficients obtained for R-P structures and double-layered perovskites ${ }^{115}$............................. 13

Figure 2.5 Crystal structure of $\mathrm{LnBaCo}_{2} \mathrm{O}_{5.5}$ with Pmmm space group, the Wyckoff oxygen ion 116 14

Figure 2.6 Defect energies (eV) of different oxygen vacancies for the $\mathrm{LnBaCo}_{2} \mathrm{O}_{5.5}$ double perovskites ${ }^{116}$ 14

Figure 2.8 Mechanisms thought in governing oxygen reduction in SOFC cathodes. Phases $\alpha, \beta$, and $\gamma$ refer to the electronic, gas, and ionic phases respectively ${ }^{10}$. 18

Figure 2.9 a) Impedance of a single silver droplet solidified on $\mathrm{YSZ}$, measured at $700{ }^{\circ} \mathrm{C}$ in oxygen, b) interpretation of the impedance in terms of bulk adsorption and transport on oxygen in silver ${ }^{135}$... 19

Figure 2.10 Scheme of the reaction mechanism in Adler's model. 20

Figure 2.11 Plot of measured (circles) and calculated (squares) complex impedance of a symmetric cell in different $P 02$ at $700{ }^{\circ} \mathrm{C}$. 22

Figure 2.12 Sketch of Svensson's model considering chemical and electrochemical reactions. . 23 Figure 2.13 Cross-sectional scheme of the physical structure and chemical reactions occurring at porous SOFC cathode. 25

Figure 2.14 Possible oxygen reduction processes by Liu et al ${ }^{151}$. 27 
Figure 2.15 Schemes of electron and ion transfer reactions at (a) metal/liquid electrolyte and (b) MIEC/solid electrolyte interfaces. 31

Figure 2.16 Sketch of Nyquist plots of MIEC electrodes, equivalent circuit, and the ORR process. 32

Figure 2.17 Scheme of the 2-D model (a) patterned electrode array (b) symmetric 2-D crosssectional model domain. 33

Figure 4.1 Schematic representation of the ECR set-up 1. Mass flow controller, 2. Four-way valve, 3. Furnace, 4. Oxygen analyzer, 5. Nano-voltmeter and current source meter, 6. Computer, 7. Three-way valve. 50

Figure 5.1 XRD patterns a) LnBCO materials calcined in nitrogen, b) compatibility with GDC.55 Figure 5.2 XRD patterns of LnBCO calcined in air. 55

Figure 5.3 Electrical conductivities of LnBCO oxides. a) PBCO, b) LBCO. .......................... 57

Figure 5.4 Raman Spectra of the PBCO oxide. ............................................................. 58

Figure 5.5 SEM morphology of a) the cross-section, b) the porous electrode of the PBCO/GDC/PBCO symmetrical cell. 59

Figure 5.6 Nyquist plot of LnBCO symmetrical cells from $700{ }^{\circ} \mathrm{C}$ to $500{ }^{\circ} \mathrm{C}$. a) $\mathrm{PBCO}$, b) NBCO, c) YBCO, d) LBCO. 63

Figure 5.7 Symmetrical cell of YBCO tested in different oxygen partial pressure: a) Nyquist plots of impedance at $700{ }^{\circ} \mathrm{C}$ and fitting line. b) Arrhenius plot and activation energy of the total cathode polarization resistance. c) Polarization resistance as a function of partial pressure and reaction order. d) $C_{\text {chem }}$ as a function of partial pressure. 64 
Figure 5.8 Nyquist plots of PBCO three-electrode cells. a) tested in $0.1 \mathrm{~V}$ cathodic overpotential. b) and c) comparison between $0.1 \mathrm{~V}$ cathodic polarization and OCV conditions. d) derived activation energy. 66

Figure 6.1 X-ray diffraction patterns of PBCO: (a) pristine tetragonal, (b) pristine orthorhombic.

Figure 6.2 Neutron Powder diffraction for two as-synthesized PBCO in 1\% air......................... 71

Figure 6.3 Room temperature neutron powder diffraction with TOF and fitting........................ 72

Figure 6.4 SAED patterns observed for $\mathrm{PrBaCo}_{2} \mathrm{O}_{5.5}$. The [100] zone axis patterns are displayed in (a) and (b) with two different diffraction domains I and II. Oriented domains (DIII) can be revealed on the zone axis [210] in (c). Supplementary weak spots can be observed in (d). e) HREM image. 75

Figure 6.5 Typical HREM images and the corresponding SAED patterns obtained along the [100] direction. 76

Figure 6.6 SAED patterns obtained along the [100] direction in a). b), c), and d) HREM of PBCO calcined in air. 78

Figure 6.7 Weight loss of PBCO in different oxygen atmospheres below $500{ }^{\circ} \mathrm{C}$. 79

Figure 6.8 Oxygen non-stoichiometry values obtained from TG results. 80

Figure 6.9 Relation between $R 2 \ln P 02$ and $1 T$ with $\delta$ as a parameter: a) tetra- $\mathrm{PBCO}, \mathrm{b})$ orthPBCO. From the slope of the plots, $\triangle H O$ is calculated. 82

Figure 6.10 Relation between $R T 2 \ln P 02$ and $T$ with $\delta$ as a parameter: a) tetra $\mathrm{PBCO}, \mathrm{b}$ ) orth PBCO. From the slope of the plots, $\Delta S O$ is calculated. 83

Figure 7.2 Electrical conductivity relaxation of pristine tetragonal and orthorhombic PBCO with time. 89 
Figure 7.3 Oxygen chemical diffusion coefficient and surface exchange coefficient for the Assynthesized tetragonal $\mathrm{PBCO}$ and orthorhombic $\mathrm{PBCO}$ as a function of temperature

Figure 7.4 SEM morphology of a) the cross-section, b) the porous electrode of the $\mathrm{PBCO} / \mathrm{GDC} / \mathrm{PBCO}$ symmetrical cell. 96

Figure 7.5 EIS profiles of $\mathrm{PrBaCo}_{2} \mathrm{O}_{6-\delta} / \mathrm{GDC}$ half cells: a) tetragonal $700{ }^{\circ} \mathrm{C}$, b) tetragonal 550 ${ }^{\circ} \mathrm{C}, \mathrm{c}$ ) orthorhombic $700{ }^{\circ} \mathrm{C}$, d) orthorhombic $550{ }^{\circ} \mathrm{C}$ 98

Figure 7.6 Reaction orders from the overall polarization resistance: a) tetragonal $\mathrm{PBCO}, \mathrm{b}$ ) orthorhombic PBCO. 102 Figure 7.7 Temperature dependence of activation energy: a) tetragonal PBCO/GDC cells, b) orthorhombic $\mathrm{PBCO} / \mathrm{GDC}$ cells. 102 


\section{List of Tables}

Table 2.1 Summary of oxygen self-diffusion and oxygen surface exchange data and overall conductivity for different SOFC cathode materials. 12

Table 2.2 Electrode processes assigned to each elementary step in surface and bulk pathways ${ }^{127}$.

Table 5.1 The refinement structure parameters for PBCO calcined in air. 54

Table 5.3 Comparison of polarization resistance of ceramic oxide cathodes in air. 60

Table 6.1 Lattice parameters evolution of pristine tetragonal and orthorhombic PBCO. 70

Table 7.1 oxygen transport parameters for as-synthesized tetragonal PBCO and..... 89 as-synthesized PBCO in air. 89

Table 7. 2 oxygen transport parameters for as-synthesized tetragonal PBCO and as-synthesized PBCO in $1 \%$ oxygen. 90

Table 7.3 The comparison of enthalpy for oxygen migration, formation between pristine tetragonal and orthorhombic PBCO. 95

Table 7.4 Summary of values related to tetragonal $\mathrm{PrBaCo} 2 \mathrm{O} 6-\delta / \mathrm{GDC}$ EIS profiles. 100 


\section{List of Symbols and Notations}

\begin{tabular}{|c|c|}
\hline SOFC & Solid oxide fuel cell \\
\hline LSM & Lanthanum strontium manganite \\
\hline TEC & Thermal expansion coefficient \\
\hline LSCF & Lanthanum strontium cobalt ferrite \\
\hline YSZ & Yttria-stabilized zirconia \\
\hline GDC & Gadolinium-doped Ceria \\
\hline MIEC & Mixed ionic and electronic conductor \\
\hline 3РB & Triple phase boundary \\
\hline $2 \mathrm{~PB}$ & Two-phase boundary \\
\hline ORR & Oxygen reduction reaction \\
\hline ECR & Electrical conductivity relaxation \\
\hline IEDP & Isotope exchange depth profiling \\
\hline MD & Molecular dynamics \\
\hline DFT & Density functional theory \\
\hline PBCO & Praseodymium barium cobalt oxide \\
\hline EIS & Electrochemical impedance spectroscopy \\
\hline $\mathrm{e}^{-}$ & Electron \\
\hline $\mathrm{V}_{\mathrm{O}}^{*}$ & Oxygen vacancy \\
\hline $\mathrm{O}_{0}^{\mathrm{x}}$ & Lattice oxygen \\
\hline$P_{\mathrm{O}_{2}}$ & Oxygen partial pressure \\
\hline TEC & Thermal expansion coefficient \\
\hline LSGM & Lanthanum strontium gallate magnesite \\
\hline $\mathrm{BSCF}$ & Barium strontium cobalt ferrite \\
\hline $\mathrm{LnBCO}$ & Lanthanide barium cobalt oxide \\
\hline LBCO & Lanthanum barium cobalt oxide \\
\hline YBCO & Yttrium barium cobalt oxide \\
\hline NBCO & Neodymium barium cobalt oxide \\
\hline $\mathrm{OCV}$ & Open circuit voltage \\
\hline$\sigma$ & Conductivity \\
\hline
\end{tabular}




$\begin{array}{ll}D^{*} & \text { Oxygen self-diffusion } \\ E_{a, D} & \text { Oxygen bulk diffusion activation energy } \\ E_{a, k} & \text { Oxygen surface exchange activation energy } \\ Z & \text { Total cell impedance } \\ R_{\text {chem }} & \text { CharaTECristic resistance } \\ t_{\text {chem }} & \text { Relaxation time } \\ C_{\text {gas }} & \text { Effective gas diffusion capacitance } \\ \text { LSC } & \text { Lanthanum strontium cobalt } \\ l_{\delta} & \text { Characteristic length } \\ O_{\text {ads }} & \text { Adsorbed oxygen atom } \\ \mathrm{h} & \text { Hole } \\ \text { ads } & \text { adsorbed site } \\ f & \text { Thermodynamic factor } \\ \varepsilon & \text { Porosity } \\ a & \text { Specific surface area } \\ V_{\mathrm{m}} & \text { Molar volume } \\ D_{\text {chem }} & \text { Bulk chemical diffusion } \\ k_{\mathrm{ex}} & \text { Surface oxygen exchange } \\ \mu_{\mathrm{O}} & \text { Chemical potential of oxygen } \\ \mu_{\mathrm{O}}^{\circ} & \text { Equilibrium chemical potential with 1 bar oxygen } \\ h_{\mathrm{O}} & \text { Partial molar enthalpy of oxygen } \\ h_{\mathrm{O}}^{0} & \text { Equilibrium partial molar enthalpy of oxygen } \\ s_{\mathrm{O}} & \text { Partial molar entropy of oxygen } \\ s_{\mathrm{O}}^{0} & \text { Equilibrium partial molar entropy of oxygen } \\ \Delta H_{\mathrm{m}} & \text { Migration enthalpy } \\ \Delta S_{\mathrm{m}} & \text { Migration entropy of } \\ \Delta H_{\mathrm{f}} & \text { Formation enthalpy } \\ \Delta S_{\mathrm{f}} & \end{array}$




\section{Introduction}

Peoples' lives improve at the expense of increasing consumption of energy and thus severe environmental pollution. Alternative energy technologies such as solar, wind, nuclear, fuel cells, high energy density batteries, and supercapacitors are being pursued intensively to address the above concerns and problems. Among them, SOFC has attracted an intensive study for its exceptionally high electrical efficiency (> $50 \%$ ) through direct conversion from chemical energy to electrical energy. SOFC has a wide variety of applications, from use as auxiliary power units in vehicles to stationary power generation stacks ${ }^{1,2}$. SOFC power stacks can increase efficiency by using the heat given off by the electrochemical oxidation within the fuel for the steam reforming process ${ }^{3}$. Additionally, solid fuels such as coal and biomass may be gasified to form syngas which is suitable for fueling SOFC ${ }^{4}$. Low-temperature SOFC $\left(600{ }^{\circ} \mathrm{C}\right)$ is one current research direction. Low-temperature systems can reduce costs by reducing insulation, materials, start-up, and degradation-related costs ${ }^{5}$. However, the deactivation of the electrode especially the cathode side is an issue when lower down the operating temperature ${ }^{6}$.

Perovskite materials have been widely served in cathode components due to their excellent electronic or both electronic and ionic conductivities. It is common sense that polarization resistance on the cathode constitutes a large portion of the whole SOFC resistance ${ }^{7}$. Since the oxygen gas needs to be reduced and transported across the gas/cathode, bulk cathode, cathode/electrolyte, and the gas/cathode/electrolyte interfaces to the anode side. Perovskite LSM with its high electrical conductivity and matched thermal expansion coefficient (TEC) with yttria-stabilized zirconia (YSZ) electrolyte has been used as a commercialized high-temperature SOFC cathode. Mixed ionic electronic conductor (MIEC) LSCF with high electronic and good ionic conductivity has been used as intermediate temperature SOFC cathode. The low ionic 
conductivity limits the utilization of LSM cathode in low to intermediate temperature SOFC. The related low surface chemical surface exchange rate on LSCF cathode needs to be addressed. Besides the most commonly used LSM and LSCF cathodes, series of double perovskite materials have been examined providing their prominent electro-catalytic performance on oxygen ${ }^{8}$. High oxygen vacancy concentration, shorter metal-oxygen bond length, and the anisotropic of oxygen transport between ab plane and $\mathrm{c}$ axis have been confirmed to contribute to the considerable electro-catalytic performance of double perovskite $\mathrm{LnBaCo}_{2} \mathrm{O}_{6-\delta}(\mathrm{LnBCO}){ }^{9}$. However, there lacks of study regarding the structure stability, oxygen transport, and oxygen reduction reaction mechanism of the double perovskite materials. They need to be studied to explain the high performance and propose a modification path leading to the commercial use of these cathodes.

The cathode reaction mechanism has been focused on LSM/YSZ and LSCF/GDC for nearly three decades through either experiment or modeling work ${ }^{10}$. Charge transfer at gas/electrode/electrolyte 3PB and surface chemical exchange at gas/electrode 2PB determined the overall oxygen reduction reaction (ORR) on the cathodes. But the results cannot be interpreted into double perovskite oxide cathode directly. Firstly, the structure of the double perovskite materials differs from the above materials in a degree of order, which will lead to the priority transport ways of oxygen ions. It is reported that oxygen transport through the ab plane is faster than through the $\mathrm{c}$ axis ${ }^{11}$. The remarkable oxygen bulk diffusion coefficient attributed to the high oxygen vacancy concentration can lead to the extension of the reaction zone from the $3 \mathrm{~PB}$ area to the gas/electrode $2 \mathrm{~PB}$ zone ${ }^{12}$. Secondly, there are dead terminators for oxygen vacancy hopping. Oxygen vacancy will associate with the antisite defects and form positively charged $\left\{\mathrm{Ba}_{\mathrm{Ln}}^{\prime}-\mathrm{V}_{\mathrm{O}}^{\prime}\right\}$ and charge-neutral $\left\{\mathrm{Ba}_{\mathrm{Ln}}^{\prime}-\mathrm{V}_{\mathrm{O}}^{*}-\mathrm{Ba}_{\mathrm{Ln}}^{\prime}\right\}^{\times}$clusters ${ }^{13}$. This will result in the selectivity of oxygen adsorption on the lattice. In addition, phase transition of double perovskite 
materials may incur lattice parameter expansion, oxygen vacancy order/disorder transition, which will affect oxygen surface exchange and bulk diffusion processes ${ }^{9}$.

Considerable research on oxygen transport performance and ORR on the cathode is either through experiment technique (ECR, IEDP, et al) or modeling (MD, DFT, ab initial). While experiment technique is limited to dense electrodes, which missed the practical porous electrodes. Besides, it is still not clear as to the details of the ORR rate-determining steps. It is possible to address the issues of porous electrodes if referring to the characterization skills for a catalyst. Therefore, a new vision that combines TGA, ECR, NPD, and EIS techniques may help understand the ORR on the cathode and further improve the activation of the cathodes at a lower temperature.

In the present study, $\mathrm{LnBCO}\left(\mathrm{PrBaCo}_{2} \mathrm{O}_{6-\delta}, \mathrm{NdBaCo}_{2} \mathrm{O}_{6-\delta}, \mathrm{YBaCo}_{2} \mathrm{O}_{6-\delta}\right.$, and $\left.\mathrm{La}_{0.5} \mathrm{Ba}_{0.5} \mathrm{CoO}_{3-\delta}\right)$ double perovskites with prominent electric and ionic conductivity, excellent oxygen transport performance are chosen as the potential cathodes. The electrochemical performance selects $\mathrm{PrBaCo}_{2} \mathrm{O}_{6-\delta}(\mathrm{PBCO})$ as a promising cathode. The research is conducted on the structure evolution of two different pristine PBCO (tetragonal and orthorhombic), thermodynamic property comparison between them, combined effects of the above two terms to oxygen transport in dense $\mathrm{PBCO}$, and oxygen reduction reaction on porous $\mathrm{PBCO}$ electrode, both experimentally and analytically. 


\section{Literature Review}

\subsection{SOFCs Cathode Materials}

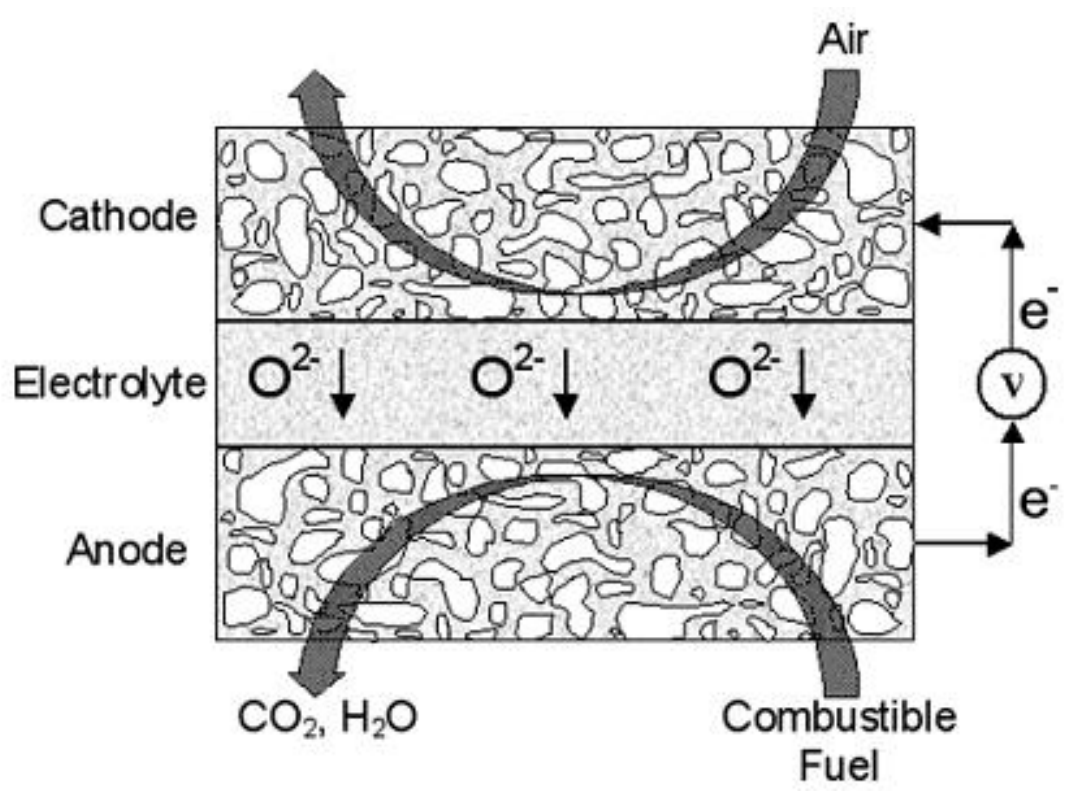

Figure 2.1 Schematic diagram of SOFC ${ }^{14}$.

Solid oxide fuel cells are energy conversion devices that produce electricity by electrochemically combining a fuel and an oxidant across an ionically conductive oxide electrolyte ${ }^{15}$. At present, the typical SOFCs consist of YSZ electrolyte, Ni/YSZ porous anode, and LSM porous cathode. Figure 2.1 illustrates the basic operating mechanism of SOFC. Oxygen is reduced to oxygen ions at the cathode and then transported to the anode through the electrolyte. Fuel is then catalytically oxidized at the anode by oxygen ion. The overall cathode reaction can be written as

$$
\frac{1}{2} \mathrm{O}_{2}+2 \mathrm{e}^{-}+\mathrm{V}_{\mathrm{O}}^{*}=\mathrm{O}_{\mathrm{O}}^{\mathrm{x}}
$$

With this simple equation, the following features must be satisfied for SOFC cathodes ${ }^{16}$ :

- Adequate electronic and ionic conductivity. 
- High catalytic activity for oxygen reduction.

- Chemical stability and relatively low interactions with the electrolyte.

- High compatibility with other cell components (electrolyte, interconnect, sealant).

Despite the high conversion efficiency of solid oxide fuel cells, the historic cell performance and stability have limited its commercial applications ${ }^{17,18}$. A substantial amount of work has been done on electrode reaction mechanism and how and why electrode performance changes with cell operation conditions ${ }^{10}$. For example, the substitute of LSM cathode with LSCF MIEC cathode introduced bulk ionic transport, then oxygen can be reduced to $\mathrm{O}^{2-}$ over the electrode surface, thereby extending the size of the active region and improving the kinetics at temperatures below $800{ }^{\circ} \mathrm{C}^{19}$. New cathodes have been investigated since oxygen reduction is generally considered to be much more difficult to be activated than hydrogen oxidation when temperature lowered to $600{ }^{\circ} \mathrm{C}{ }^{20-28}$. Compared to the traditional LSM and LSCF cathodes, the newly developed cathodes tried to improve the activation of cathodes at lower temperatures and address the long-term degradation issue.

\subsection{1 $\mathrm{ABO}_{3}$ Perovskite Oxides}

During the early stage of SOFC development, platinum and some other noble metals were used as the cathode material ${ }^{29-31}$. However, platinum is expensive and its compatibility with the electrolyte is not so good due to the TEC mismatch between metal and the ceramic electrolyte. Less expensive perovskites, with their diversity in structure, chemical composition, and high chemical stability have attracted much interest ${ }^{32-36}$. Particularly, the application of Co- and Mncontaining perovskites as cathodes has been extensively studied for reasons of their high electrical conductivity and catalytic activity for oxygen dissociation ${ }^{37-40}$. 


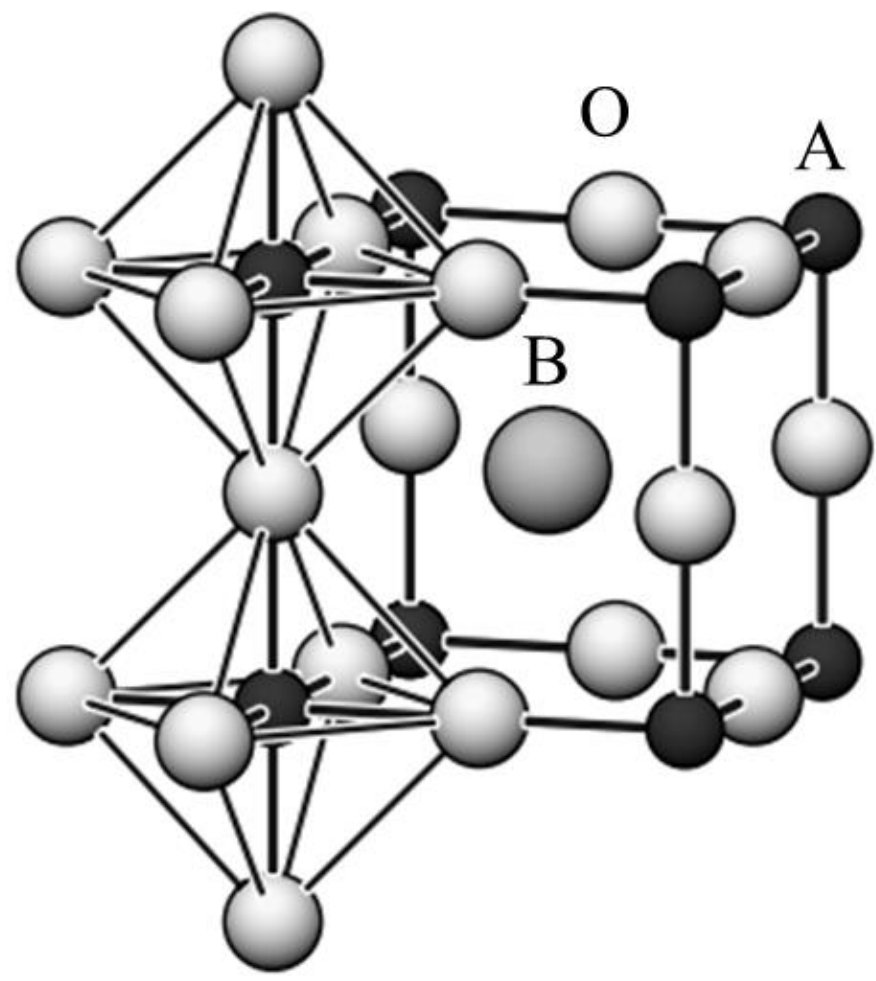

Figure 2.2 Schematic diagram of the $\mathrm{ABO}_{3}$ perovskite structure.

Figure 2.2 reveals the lattice structure of $\mathrm{ABO}_{3}$ perovskite. In general, in the perovskite-type structure, the B site element is usually a transition metal with small radii closely bonded to six oxygen atoms with a strong covalent nature, while the A site rare earth element coordinates to 12 oxygen ions with a strong ionic conductive nature ${ }^{41}$. Considering the flexible choices at A and $\mathrm{B}$ sites concerning not only wide size variety but also different valence combinations, perovskite oxides are largely adjustable in terms of the electronic, ionic conductivities, TEC, catalytic properties, chemical stability, etc ${ }^{42}$. The big tolerance of oxygen vacancy in the lattice entitles such structure to attract transport property ${ }^{43}$. The stability of perovskite towards $P_{\mathrm{O}_{2}}$ mainly depends on the A-site elements using rare-earths ${ }^{19}$.

$\mathrm{La}_{1-\mathrm{x}} \mathrm{Sr}_{\mathrm{x}} \mathrm{MnO}_{3 \pm \delta}$ (LSM) is one of the most widely used and investigated cathode perovskite materials in SOFC. Sr-doped lanthanum perovskites show both large oxygen excess under 
oxidizing atmosphere and large oxygen-deficiency when in reducing gas atmospheres ${ }^{44}$. In the oxygen-deficient region, oxygen vacancies are the main defects while in the oxygen excess region metal vacancies are the main defects ${ }^{45}$. Despite adequate electronic conductivity and the good compatibility with YSZ electrolyte ${ }^{46}$, the oxygen ionic conductivity and its oxygen tracer diffusion coefficient are undesirably low ${ }^{47}$. This limits the application of LSM cathodes to SOFC operating at above $800{ }^{\circ} \mathrm{C}$.

The mixed ionic and electronic conductive perovskite $\mathrm{La}_{1-\mathrm{x}} \mathrm{Sr}_{\mathrm{x}} \mathrm{Co}_{1-\mathrm{y}} \mathrm{Fe}_{\mathrm{y}} \mathrm{O}_{3 \pm \delta}$ (LSCF) is another candidate material for SOFC cathodes. The cell performance can be greatly improved at intermediate temperature because of the high ionic conductivity ${ }^{48}$. However, the compatibility of LSCF with YSZ electrolyte is an issue, due to the higher thermal expansion coefficient (TEC) relative to the TEC of the YSZ electrolyte ${ }^{49}$. GDC and LSGM have been substituted for YSZ as an electrolyte for intermediate SOFC, due to their higher TEC ${ }^{50}$.

\subsubsection{Ordered Double-Layer Perovskite Oxides}

With matched electrolytes, a series of high oxygen transport performance cathodes were studied and developed. Among them, $\mathrm{Ba}_{0.5} \mathrm{Sr}_{0.5} \mathrm{Co}_{0.8} \mathrm{Fe}_{0.2} \mathrm{O}_{3-\delta}$ and double perovskite $\mathrm{LnBaCo}_{2} \mathrm{O}_{6-\delta}$ $(\mathrm{LnBCO})(\mathrm{Ln}=\mathrm{La}, \mathrm{Sm}, \mathrm{Pr}, \mathrm{Gd}, \mathrm{Ho}$, et al) outstand with their excellent electro-catalytic activity to ORR at intermediate operating temperatures ${ }^{51,52}$. Figure 2.3 shows the lattice structure of LnBCO materials with single cubic, orthorhombic and tetragonal structures ${ }^{53,54}$. They can be represented by the stacking sequence of $\left|\mathrm{LnO}_{\delta}\right| \mathrm{CoO}_{2}|\mathrm{BaO}| \mathrm{CoO}_{2}$ layers. The orthorhombic and tetragonal structures consist of a cation-ordered sub-lattice with oxygen vacancy located in layers ${ }^{55}$. LnBCO materials were first being studied due to their magnetic properties with thermo- 
driven phase transition phenomena ${ }^{56-59}$. Recently, they have also received attention with excellent oxygen reduction reaction activity at low to intermediate temperature SOFC ${ }^{60-64}$.
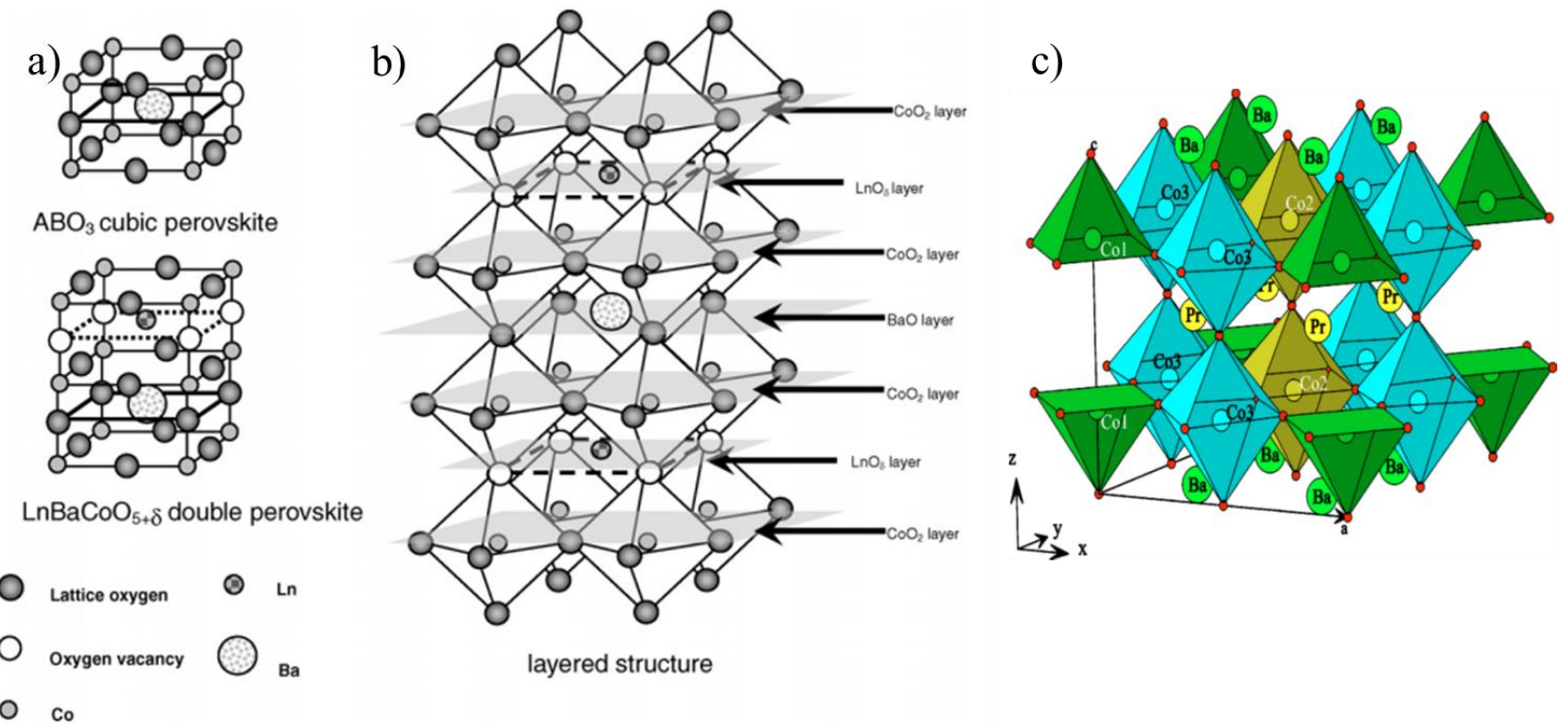

Figure 2.3 Schemes perovskite LBCO: (a) cubic, (b) orthorhombic, and (c) tetragonal ${ }^{53,54}$.

\subsection{Phase Transition of Double Perovskite $\mathrm{LnBaCo}_{2} \mathrm{O}_{6-\delta}$}

The stated $\mathrm{LnBaCo}_{2} \mathrm{O}_{6-\delta}$ orthorhombic structure materials have been investigated as SOFC cathodes. However, it conflicts with the researchers that first studied the thermodynamic property of this material ${ }^{65}$. SOFC researchers attribute the excellent oxygen transport performance of $\mathrm{LnBaCo}_{2} \mathrm{O}_{6-\delta}$ to their cation-ordered-anion-disordered structure ${ }^{66-68}$. However, condensed matter researchers assume $\mathrm{LnBaCo}_{2} \mathrm{O}_{6-\delta}$ to be a cation-ordered-anion-ordered structure, especially for $\mathrm{LnBaCO}_{2} \mathrm{O}_{5.5}{ }^{69-71}$. Under the circumstance of SOFC operating temperature, there is a controversial issue on the phase of LnBCO as it will undergo a phase transition at higher temperatures ${ }^{72-75}$. The phase transition may be an oxygen anion ordered/disordered transition, leading to the blurry of the oxygen transport performance 
explanation and mechanism analysis. Besides, phase transition may attribute to the sudden TEC change that leads to the deterioration of the electrode/electrolyte interface.

The transition-metal oxides have been attracted a considerable focus since their extended use in the field of materials science and application in energy-related realms ${ }^{76-78}$. Among them, cobalt oxides are the subject of intensive studies. In cobaltites, the spin state degree freedom of Co ions has introduced new interesting effects in the case of narrow-band oxides ${ }^{79}$. Among other families like misfit cobaltites for thermos-power applications, ${ }^{80,81}$ or mixed-valence cobalt perovskites as candidates or present charge ordering like $\operatorname{Pr}_{0.5} \mathrm{Ca}_{0.5} \mathrm{O}_{3}{ }^{82}$, layered cobaltites with general formula $\mathrm{LnBaCo}_{2} \mathrm{O}_{6-\delta}$ have been demonstrated to be complex systems with very enthralling phenomena like charge ordering ${ }^{83,84}$, metal-insulator transitions ${ }^{56,85}$, orbital order variability ${ }^{86}$, and also a large range of thermoelectric power ${ }^{87}$. After the study by Troyanchuk et al. ${ }^{85}$, the corner stonework by Maignan et al ${ }^{56}$ discovered that the oxygen content of these compounds can be controlled through different atmosphere treatments, for example, the inert gas served orthorhombic $\mathrm{LnBaCo}_{2} \mathrm{O}_{5.5}$ oxides ${ }^{88}$ and the tetragonal $\mathrm{LnBaCo}_{2} \mathrm{O}_{5.75}$ compounds obtained with air treatment ${ }^{89}$. They both have ordering in alternating layers of Ba and Ln ions and the tendency of oxygen vacancies to Ln planes forming the ordered patterns of cation sublattice.

The most alluring characters of $\mathrm{LnBaCo}_{2} \mathrm{O}_{6-\delta}$ double perovskites cobaltites in SOFC cathode application are their high oxygen vacancy concentration and the resulting excellent oxygen bulk diffusion performance. The vacancy concentration is highly related to cobalt valence. Besides, the spin-charge-orbital that Co presents can facilitate the transition between different spin states 90. The balance between the Hund's rule and the crystal field, combined with the distortion in octahedral or pyramidal coordinated oxygen domains can stabilize three different spin states: low 
spin (LS), intermediate spin (IS), and thigh spin (HS) for both $\mathrm{Co}^{3+}$ (LS with $t_{2 g}^{6}$ and $\mathrm{S}=0$, IS with $t_{2 g}^{5} e_{g}^{1}$ and $\mathrm{S}=1$, and $\mathrm{HS} t_{2 g}^{4} e_{g}^{2}$ with and $\mathrm{S}=2$ ) and $\mathrm{Co}^{4+}$ (LS with $t_{2 g}^{5}$ and $\mathrm{S}=1 / 2$, IS with $t_{2 g}^{4} e_{g}^{1}$ and $\mathrm{S}=3 / 2$, and $\mathrm{HS} t_{2 g}^{3} e_{g}^{2}$ with and $\left.\mathrm{S}=5 / 2\right)^{90}$.

The average cobalt valence, as well as the coordination of Co in either octahedral or pyramidal, is controlled through the tailoring of oxygen non-stoichiometry ${ }^{91}$. This leads to the magnetic and transport properties of these compounds being mainly dominated by the oxygen content. Take $\mathrm{YBaCo}_{2} \mathrm{O}_{5}{ }^{84}$ and $\mathrm{TbBaCo}_{2} \mathrm{O}_{5}{ }^{92}$ as examples, with higher values of oxygen deficiency, they easily exhibit cation-ordered structure. The competing ferromagnetic and antiferromagnetic interactions will cause metal-insulator and spin-state transitions in oxides with $\delta=0.556,58,59,86,93$. The metal-insulator transition is due to spin state change from $\mathrm{Co}^{3+}\left(t_{2 g}^{6}\right)$ to $\mathrm{Co}^{3+}\left(t_{2 g}^{4} e_{g}^{2}\right) \mathrm{O}_{6}$ octahedra ${ }^{70}$. For $\delta=0.5$, it is verified that oxygen vacancies are filled in empty roles of oxygen sites along the a-axis in the $\mathrm{LnO}_{\delta}$ planes. Along with this ordering, low spin states of $\mathrm{Co}^{3+}$ were found to be orderly distributed in the structure below the transition temperature and high spin states above transition temperature ${ }^{70}$. Another phenomenon that occurred for $\mathrm{LnBaCo}_{2} \mathrm{O}_{6-\delta}$ as colossal magnetoresistance materials is the ferromagnetic-antiferromagnetic (FM-AFM) competition ${ }^{94}$. Ordering oxygen in LnO layers into alternating empty and filled rows favors a strong uniaxial anisotropy of $\mathrm{Co}^{3+}$ spins. In the FM phase, Co spins align along an oxygen-chain direction forming ferromagnetic two-leg ladders, which interact with each other by the mediation of nonmagnetic Co-O layers. In contrast to FM, this coupling turns out to be AFM at low temperatures ${ }^{93}$. 


\subsection{Anisotropic Oxygen Ion Diffusion in Layered Cathodes}

MIEC materials show priorities over traditional LSM cathodes in SOFCs applications, particularly in the intermediate to low temperature range where cathode limits the cell performance. A considerable amount of layered oxide materials have been found to present remarkable properties as MIEC $^{8}$. So substantial studies have been devoted to better understand and evaluate layered ordered structures.

Ruddlesden-Popper (RP phase) series and double layer perovskite materials have been widely studied in cathode applications ${ }^{95-101}$. Table 2.1 compared some conventional layered cathodes. The anisotropy of oxygen diffusion and the existence of cation-ordered structures improve the oxygen transport properties compared to non-ordered compounds, leading to lower activation energies and excellent oxygen surface exchange properties, which turn these materials into suitable cathodes for operation at intermediate temperatures.

Ordered double perovskites with the general formula $\mathrm{AA}^{\prime} \mathrm{B}_{2} \mathrm{O}_{6-\delta}$ have been recently proposed as next-generation cathode materials for intermediate to low temperature operation ${ }^{102}$. High electronic conductivity was observed by Maignan ${ }^{56}$ et al. for these layered perovskites above the metal-insulator transition temperature. These material series are derived from $50 \%$ lower valent substitution on the A-site of a normal perovskite oxide such that the general formula can be written as $\mathrm{A}_{0.5} \mathrm{~A}^{\prime}{ }_{0.5} \mathrm{BO}_{3-\delta}{ }^{103}$. This is the formula with two cations disordered occupying the $\mathrm{A}$ sites. However, when the size difference between the two cations is significant they tend into order and the formula is rewritten as $\mathrm{AA}^{\prime} \mathrm{B}_{2} \mathrm{O}_{6-\delta}{ }^{104}$. Taskin ${ }^{105,106}$ et al. first investigated the reoxidation kinetics of $\mathrm{GdBaMn}_{2} \mathrm{O}_{6-\delta}$ in which the A cation sub-lattice can be either ordered or disordered depending upon the preparation technique employed. The result presented shows that 
the chemical diffusion coefficient of oxygen can be greatly enhanced with an A-site cation order structure. Besides, oxygen self-diffusion coefficients were derived from their chemical diffusion data using a thermodynamic enhancement factor ${ }^{107}$. Referring to these interesting works, two groups have measured the diffusion of oxygen in ordered double perovskite materials using isotope methods ${ }^{52,101}$. The measured values are shown in Figure 2.4a-b.

Table 2.1 Summary of oxygen self-diffusion and oxygen surface exchange data and overall conductivity for different SOFC cathode materials.

\begin{tabular}{|c|c|c|c|c|c|c|}
\hline & $\begin{array}{l}D^{*} / \mathrm{cm}^{2} \mathrm{~s}^{-1} \\
T=500^{\circ} \mathrm{C}\end{array}$ & $\begin{array}{r}E_{a, D} / \\
\mathrm{eV}\end{array}$ & $\begin{array}{l}k^{*} / \mathrm{cms}^{-1} \\
T=500^{\circ} \mathrm{C}\end{array}$ & $\begin{array}{l}E_{a, k} / \\
\mathrm{eV}\end{array}$ & $\begin{array}{l}\sigma / \mathrm{Scm}^{-1} \quad \mathrm{~T}=500- \\
700^{\circ} \mathrm{C}\end{array}$ & Ref. \\
\hline $\mathrm{La}_{0.8} \mathrm{Sr}_{0.2} \mathrm{MnO}_{3-\delta}$ & $4.5 \times 10^{-20}$ & 2.80 & $3.1 \times 10^{-11}$ & 1.30 & $120-130$ & $108-110$ \\
\hline $\mathrm{La}_{0.8} \mathrm{Sr}_{0.2} \mathrm{CoO}_{3-\delta}$ & $9.0 \times 10^{-14}$ & 2.22 & $2.8 \times 10^{-9}$ & 1.32 & $1500-1600$ & $\begin{array}{l}108,109,11 \\
1\end{array}$ \\
\hline $\mathrm{La}_{0.5} \mathrm{Sr}_{0.5} \mathrm{CoO}_{3-\delta}$ & $1.5 \times 10^{-10}$ & 1.41 & $3.9 \times 10^{-7}$ & 0.81 & $1300-1800$ & $\begin{array}{l}108,109,11 \\
1\end{array}$ \\
\hline $\mathrm{La}_{0.6} \mathrm{Sr}_{0.4} \mathrm{Co}_{0.2} \mathrm{Fe}_{0.8} \mathrm{O}_{3-\delta}$ & $1.2 \times 10^{-10}$ & 1.39 & $3.3 \times 10^{-9}$ & 1.60 & $250-300$ & 111,112 \\
\hline $\mathrm{Ba}_{0.5} \mathrm{Sr}_{0.5} \mathrm{Co}_{0.8} \mathrm{Fe}_{0.2} \mathrm{O}_{3-\delta}$ & $1.2 \times 10^{-7}$ & 0.5 & $1.1 \times 10^{-6}$ & 1.76 & $10-55$ & 113,114 \\
\hline $\mathrm{La}_{2} \mathrm{NiO}_{4+\delta}$ & $3.3 \times 10^{-9}$ & 0.56 & $7.0 \times 10^{-9}$ & 0.60 & $55-65$ & 95,96 \\
\hline $\mathrm{La}_{2} \mathrm{CoO}_{4+\delta}$ & $2.5 \times 10^{-8}$ & 0.12 & $3.2 \times 10^{-6}$ & 0.03 & $1-5$ & 97,98 \\
\hline $\mathrm{GdBaCo}_{2} \mathrm{O}_{6-\delta}$ & $2.8 \times 10^{-10}$ & 0.6 & $7.5 \times 10^{-8}$ & 0.81 & $550-925$ & 99,100 \\
\hline $\mathrm{PrBaCo}_{2} \mathrm{O}_{6-\delta}$ & $3.6 \times 10^{-7}$ & 0.48 & $6.9 \times 10^{-5}$ & 0.67 & $400-700$ & 101 \\
\hline
\end{tabular}



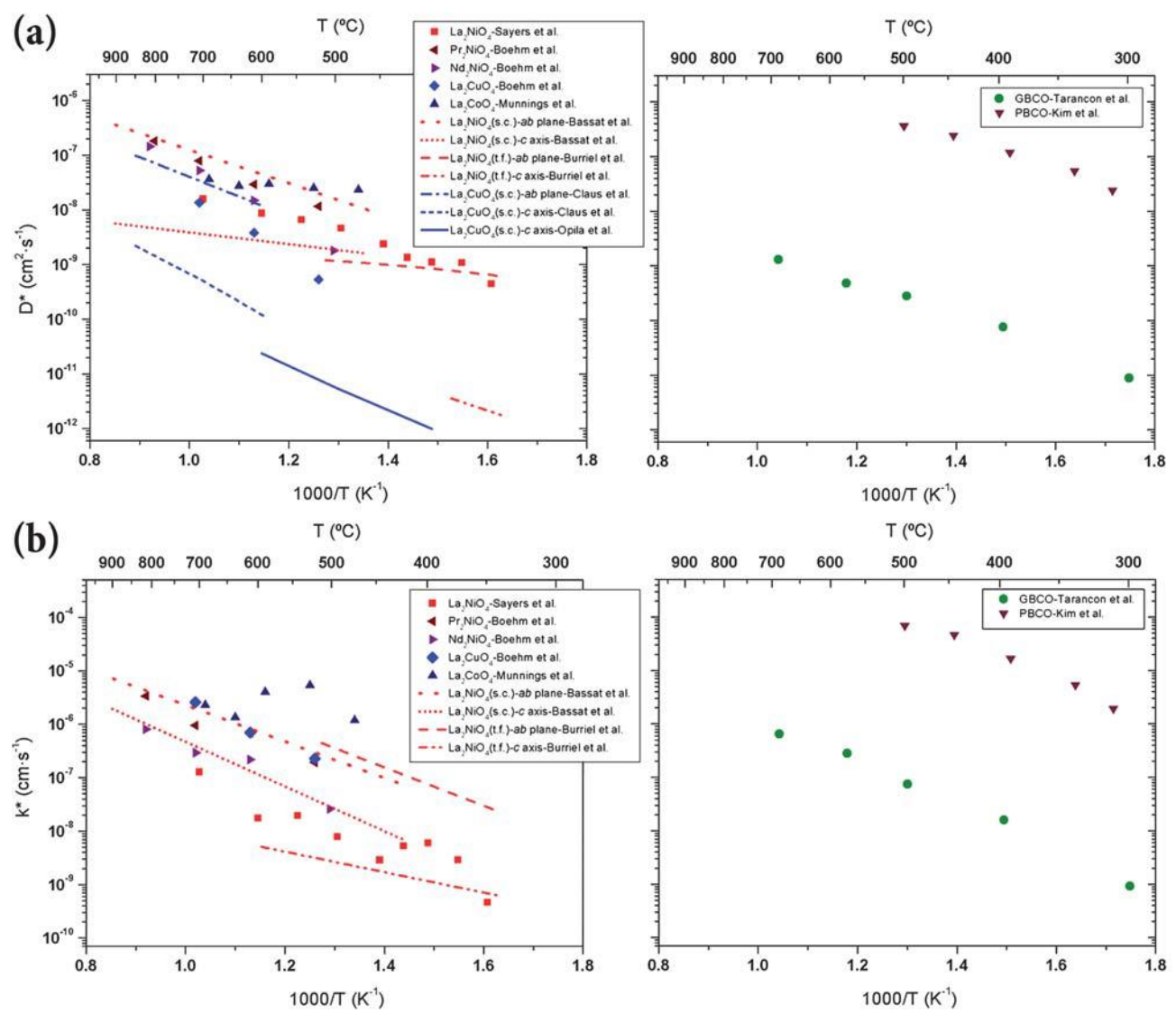

Figure 2.4 Arrhenius plot of (a) the oxygen tracer diffusion and (b) the surface exchange coefficients obtained for R-P structures and double-layered perovskites ${ }^{115}$.

For these cation-ordered double perovskites, oxygen Frenkel disorder is important since it controls the concentration of oxygen defects which are the critical factor for oxygen transport in $\mathrm{LnBaCo}_{2} \mathrm{O}_{5.5}{ }^{13}$. There are six independent oxygen sites shown in Figure 2.5. Figure 2.6 presents the defect energies of different oxygen vacancies for $\mathrm{LnBaCo}_{2} \mathrm{O}_{5.5}$ double perovskites. The lowest energy vacancy positions are the $\mathrm{O} 3$ (located in $\mathrm{LnO}_{0.5}$ layers, see Figure 2.5), O4, and $\mathrm{O} 6$ (located in the $\mathrm{CoO}_{2}$ layers) ${ }^{13}$. The $\mathrm{O} 5$ position (in the $\mathrm{CoO}_{2}$ layers) offers a low-energy vacant site only for compositions with the smallest Ln cations. The $\mathrm{O} 3$ site energy also increases consequentially with increasing Ln cation radius. The highest energy vacancy positions (more than $2-4 \mathrm{eV}$ ) are the $\mathrm{O} 1$ and $\mathrm{O} 2$ positions, which are located in $\mathrm{BaO}$ layers. This suggests that if 
oxygen transport is to occur through a vacancy hopping mechanism it will mainly be resstricted into $\mathrm{LnO}_{0.5}$ and $\mathrm{CoO}_{2}$ layers, with very limited transport in or through the $\mathrm{BaO}$ layers. This indicates the anisotropic diffusion mechanism. It is also verified by molecular dynamics calculations in $\mathrm{GdBaCo}_{2} \mathrm{O}_{6-\delta}{ }^{11,66}$.

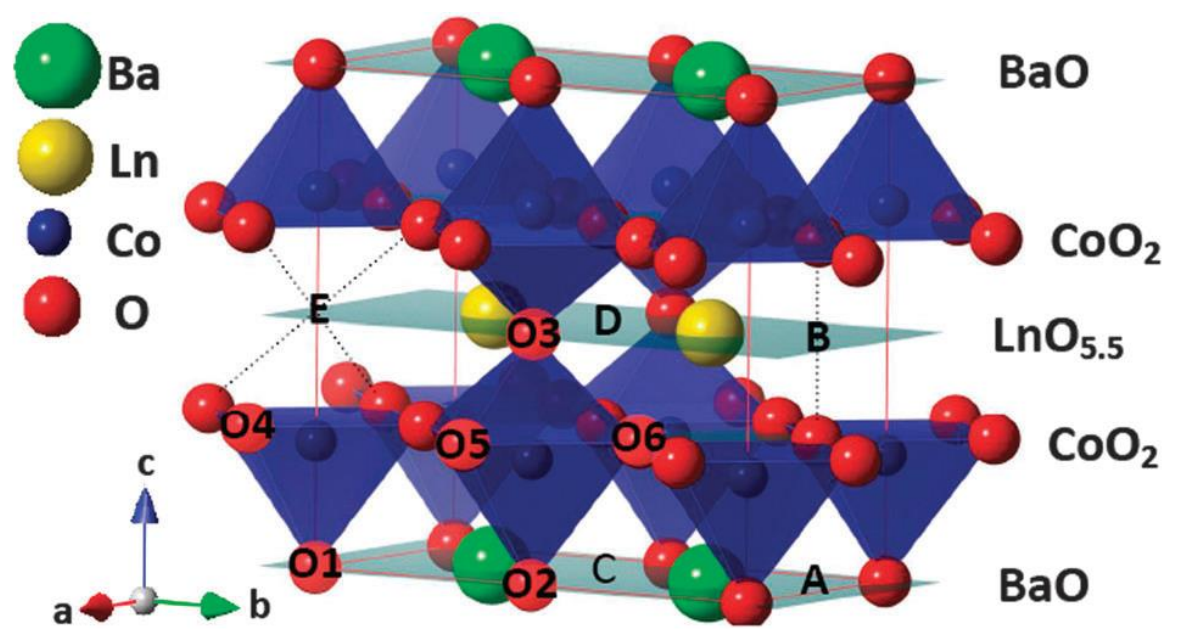

Figure 2.5 Crystal structure of $\mathrm{LnBaCo}_{2} \mathrm{O}_{5.5}$ with Pmmm space group, the Wyckoff oxygen ion ${ }^{116}$.

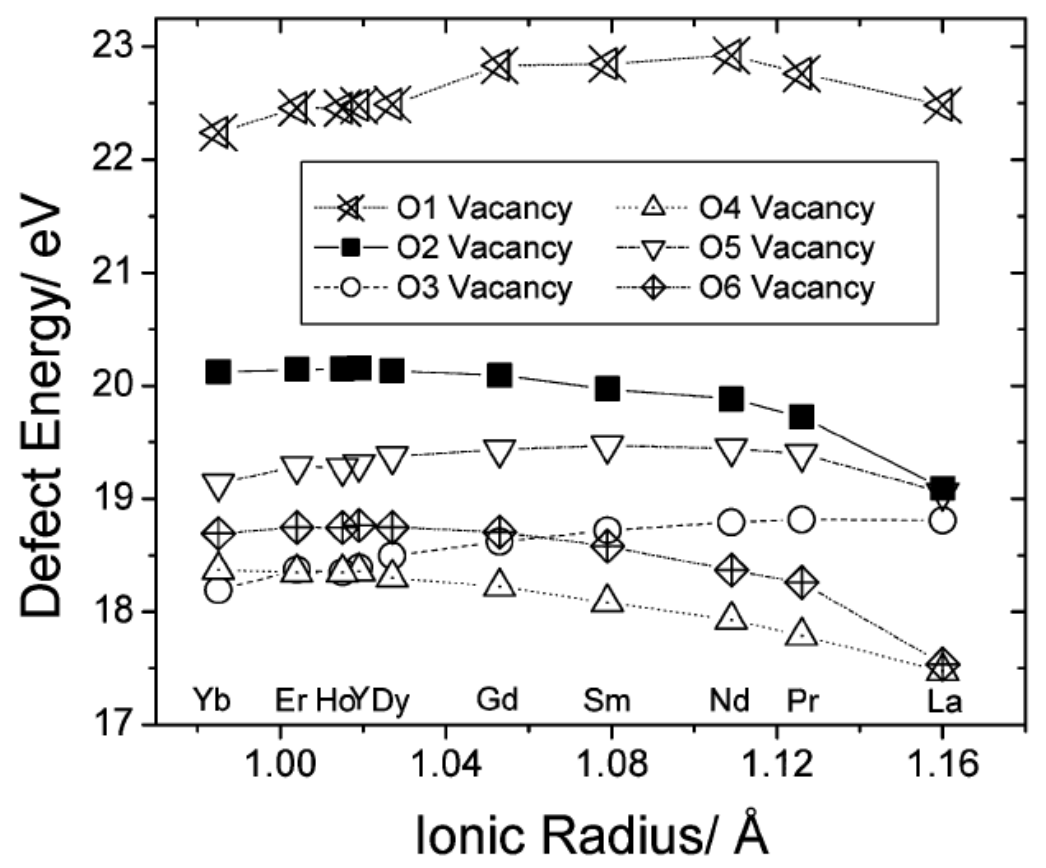

Figure 2.6 Defect energies (eV) of different oxygen vacancies for the $\mathrm{LnBaCo}_{2} \mathrm{O}_{5.5}$ double perovskites ${ }^{116}$. 
The work on $\mathrm{GdBaCo}_{2} \mathrm{O}_{5.5}$ demonstrated that oxygen transport is through a vacancy mechanism and that cation anti-site defects can strongly depress the oxygen transport ${ }^{66}$. Another conclusion of the research on $\mathrm{GdBaCo}_{2} \mathrm{O}_{5.5}$ is that high cation disorder (anti-sites) converts the oxygen transport mechanism from anisotropic to isotropic. Norby ${ }^{117}$ has evaluated the phenomenon of linked cation and anion ordering. Contrary to the belief that cation disordered structures are better oxide ion conductors, Norby proposed that materials with cation order and anion disorder exhibit high oxygen diffusivities. In fact, for $\mathrm{Gd}_{0.5} \mathrm{Ba}_{0.5} \mathrm{CoO}_{3-\delta}$ a fully disordered cation lattice, defect trapping between the oxygen vacancies and the $\mathrm{Ba}_{\mathrm{Gd}}^{\prime}$ anti-site was assumed to be responsible for the calculated low diffusivity ${ }^{66}$.

\subsection{Oxygen Reduction in the Surface Process Limited Cathode}

The cathode reaction mechanism is a critical issue for electrochemistry study in SOFC application especially at low to intermediate temperatures. Researchers ${ }^{118-120}$ have studied electrode reaction mechanisms, developed new electrode materials and characterized their microstructures, and elucidated the typical structure-property-performance relationship. The electrode crystal structure determines the intrinsic oxygen transport property. The typical SOFC cathode porosity is $\sim 30 \%$ and the thickness is $\sim 30 \mu \mathrm{m}$. Based on this, electrode performance deteriorates with time, temperature, thermal cycling, impurities, or other factors that have been investigated and mitigated.

In the traditional case of SOFC cathode, the oxygen reaction at the gas/electrode/electrolyte triple phase boundary (3PB) and the electrode performance models based on it have guided the design of electrodes ${ }^{121}$. However, the mechanistic insight in a deeper understanding of the SOFC cathode reaction is poor. The electrode reaction was separated into elementary steps, including 
the adsorption, dissociation, and reduction of oxygen at the gas/electrode, gas/electrode/electrolyte interface ${ }^{122}$. Following this work, diffusion processes were recognized limiting electrode performance and can imitate the activation behavior ${ }^{123}$. To enlarge the activation area of the cathode, bulk ionic conductive oxides are introduced, for example, La1${ }_{\mathrm{x}} \mathrm{Sr}_{\mathrm{x}} \mathrm{Co}_{1-\mathrm{y}} \mathrm{Fe}_{\mathrm{y}} \mathrm{O}_{3-\delta}(\mathrm{LSCF}){ }^{124}$. Oxygen can be reduced to $\mathrm{O}^{2-}$ over the electrode surface, thereby extending the size of the active region. A thorough elucidation includes useful information on how to improve cell performance. Limited by the development of solid-state ionic conductivity theories, researchers have matured SOFC cathode models over the past 20 years.

Aqueous solutions are the traditional domain of electrochemical system studies ${ }^{125,126}$. Therefore, generally accepted conclusions and common investigation methods of the aqueous system are the basis for SOFC cathode studies. However, the solid-state system is more complex compared with the aqueous system. First, there is a three-phase boundary (3PB), where gas molecular, cathode, and electrolyte materials meet. Meanwhile, gas/cathode and cathode/electrolyte two phase boundaries (2PB) also exist at the cathode side. The parallel 3PB and 2PB pathways provide competing routes for oxygen reduction ${ }^{127}$, see Figure 2.7. 


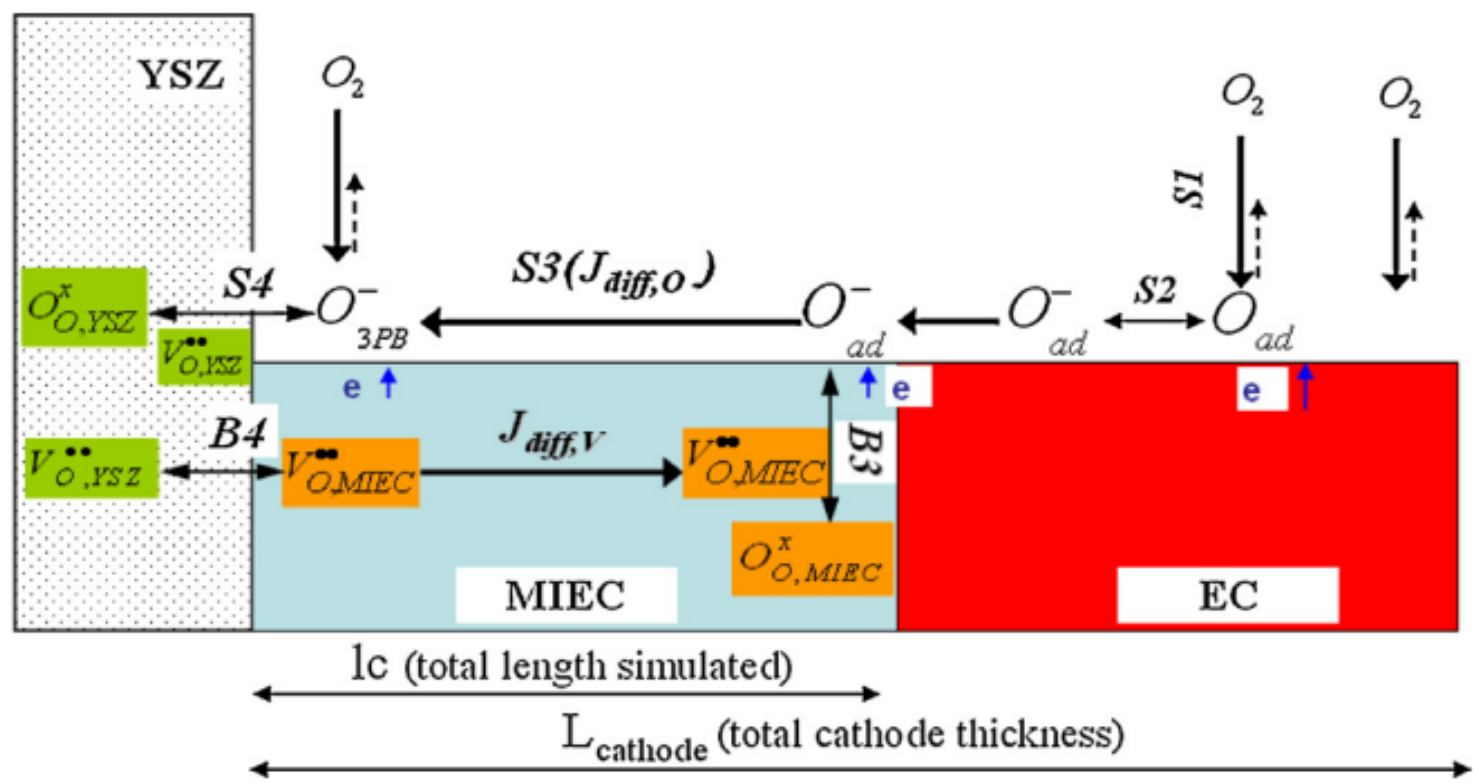

Figure 2.7 Schematic description of bi-pathway dominated oxygen reduction on MIEC cathode (LSM).

In aqueous systems, only ion diffusion occurs while for SOFC cathodes oxygen gas and ions will diffuse against the electrons ${ }^{10}$. Finally, the double layer formed on the electrode is different. For the aqueous system, usually, the inactive ions in the solution will move to the electrode surface under the electrical field. However, on the surface of the SOFC cathode, adsorbed atomic oxygen or ions will form the double layer. Figure 2.8 profiles several typical rate-determining steps in the oxygen reduction reaction ${ }^{10}$. Oxygen molecules are commonly adsorbed onto one or more solid surfaces, where they experience catalytic or electro-catalytic reduction steps to form partially or fully reduced ionic or atomic species ${ }^{128}$. These electroactive species need to transport along surfaces, interfaces, the bulk of the electrode to the electrolyte, where the fully reduced oxygen ions are being diffused to the anode side to oxidize the forming gas electrocatalytically. Among several transport pathways, the rate-determining for a certain electrode needs to be investigated in detail regarding their specific intrinsic properties, microstructure, and operating conditions. 

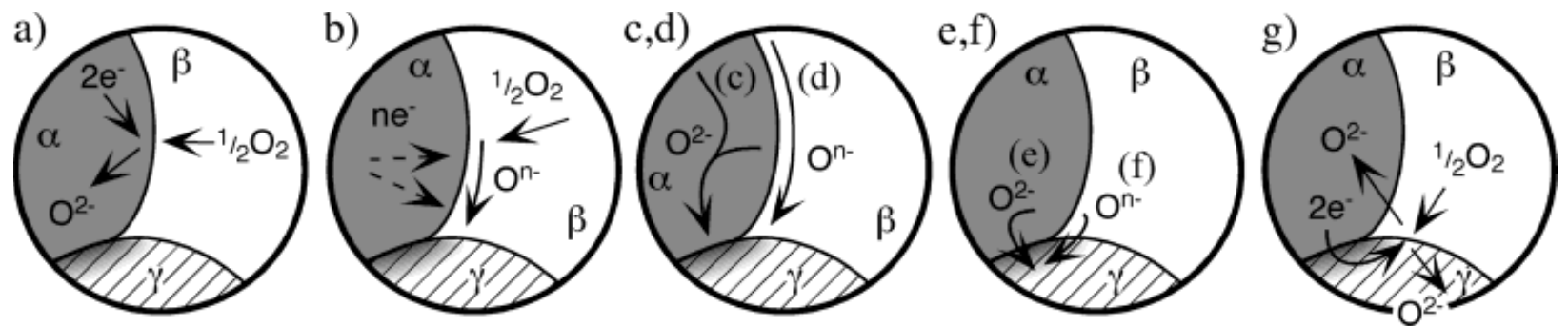

Figure 2.8 Mechanisms thought in governing oxygen reduction in SOFC cathodes. Phases $\alpha, \beta$, and $\gamma$ refer to the electronic, gas, and ionic phases respectively ${ }^{10}$.

One of the most widely experimental techniques to analyze gas diffusion electrode is electrochemical impedance spectroscopy (EIS) ${ }^{129}$. It identifies the reaction steps via a time scale calculated from the characteristic frequency 130,131 . The general analysis of impedance is by equivalent circuit fitting. The high capacitance from the low-frequency profile cannot explain the traditional double layer polarization resistance at an electrode/electrolyte interface ${ }^{132}$. The lowfrequency arcs are supposed to oxygen concentration changes in the gas/electrode two-phase boundary, indicating a concentration over-potential instead of kinetics resistance ${ }^{133}$.

Silver on the YSZ system was adopted to study the diffusion-limited kinetics of oxygen reaction ${ }^{134}$. The impedance of silver electrodes was tested as a function of $\mathrm{T}\left(600{ }^{\circ} \mathrm{C}\right.$ to $\left.800{ }^{\circ} \mathrm{C}\right)$, $P_{\mathrm{O}_{2}}(0.01$ to $1.0 \mathrm{~atm})$. Figure 2.9a presents a Nyquist plot of the impedance under one set of conditions. The profile is fitted into two parts a very low-frequency one $(0.01$ to $1 \mathrm{~Hz})$ and another one $(\sim 10 \mathrm{~Hz})$. The author stated that the low-frequency polarization resistance is mainly associated with adsorption and diffusion inside the silver droplet. The proposed mechanism is demonstrated in Figure 2.9b. It is explained that atomic oxygen was first dissolved in the silver to be reduced to oxygen ions and then transported into the silver/YSZ interface. The depletion of oxygen in the metal close to the silver/YSZ interface creates a chemical potential for dissolved oxygen to diffuse to the interface from the bulk of the silver, results in the diminishing of the 
dissolved oxygen near the silver/gas interface. Depletion of oxygen in silver with relate to gas facilitate the dissociative adsorption of gaseous $\mathrm{O}_{2}$ into the silver. Consequently, gas diffusion is proved to mainly contribute to the limits of the overall reaction. This is the only mechanism that elucidates the large capacitance of the electrode (larger than $1 \mathrm{~F} / \mathrm{cm}^{2}$ ). The fact is that the reaction is rather co-limit by several steps than just one. The $45^{\circ}$ relationship is not restricted to the finite diffusion limit. It can also be a semi-infinite diffusion limit. It is an indicator of the gas diffusion limit.
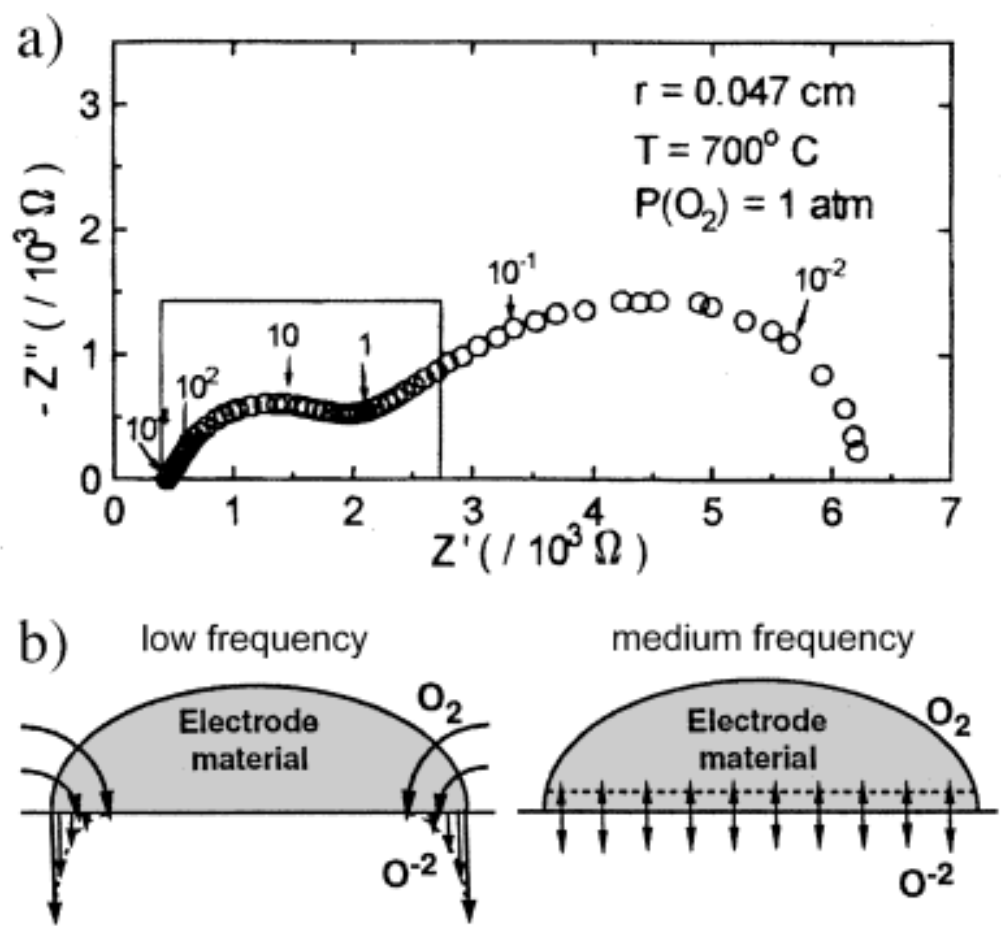

Figure 2.9 a) Impedance of a single silver droplet solidified on YSZ, measured at $700{ }^{\circ} \mathrm{C}$ in oxygen, b) interpretation of the impedance in terms of bulk adsorption and transport on oxygen in silver ${ }^{135}$.

SOFC cathode models can be categorized by dimensions, model setup method, the scale of models. There are 1-D, 2-D, and 3-D models according to the dimension ${ }^{127,136,137}$. Further, some reported SOFC cathode models based on the theory viewpoint ${ }^{138}$ while others based on experimental data ${ }^{139}$. For example, some studies on the ORR at 3PB had been done based on the 
SEM morphology ${ }^{140}$. Finally, SOFC cathode models can be divided into micro-models ${ }^{141}$ and macro models ${ }^{142}$ according to different investigation scales. The former studies the detailed cathode reaction elementary steps and the latter mainly considered the entire effect of the factors such as porosity, gas flow rate, temperature, etc. Since our work focused on micro-scale oxygen transportation kinetics, the development of SOFC cathode micro-models will be reviewed. Thus far, the most controversial issue is whether ORR is controlled by a chemical process or an electrochemical process.

\subsubsection{Pure Chemical Process}

Adler developed a model which assumes the SOFC cathode reaction as a pure chemical process in 1996, as shown in Figure 2.10, Adler et al ${ }^{143}$ stated that the overall cathode reaction occurred via three reactions: (1) charge transfer of oxygen vacancies across the cathode/electrolyte interface; (2) charge transfer of electrons across the current collector/cathode interface; (3) chemical exchange of oxygen at the gas/cathode surface. This model is valid for mixed conductors LSC.

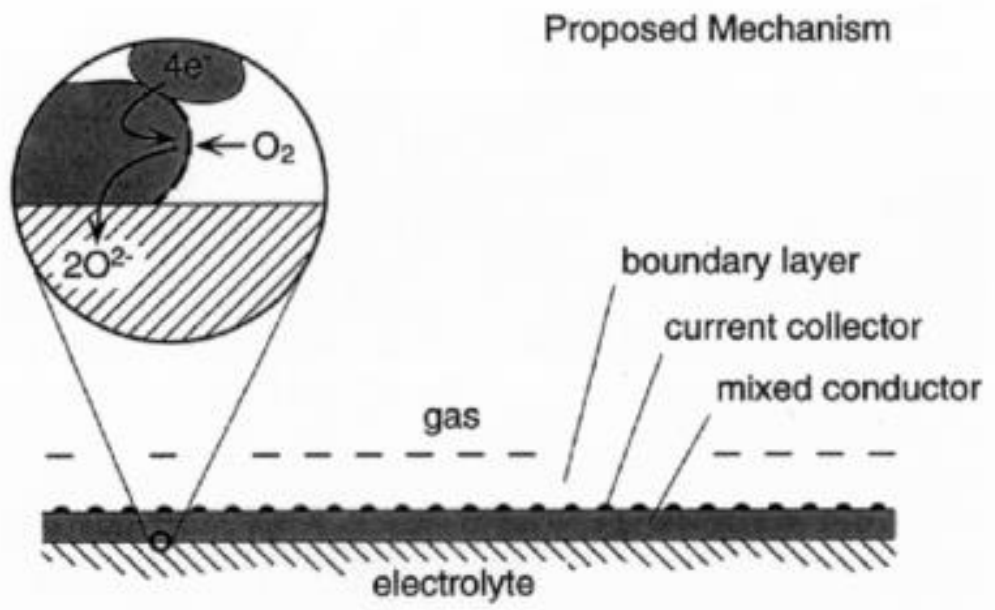

Figure 2.10 Scheme of the reaction mechanism in Adler's model. 
Only the oxygen diffusion and exchange at the electrode/gas interface contribute to the cathode kinetics in this model. To clarify the chemical contribution, either the surface exchange and solid-state diffusion or the gas phase diffusion was considered as the rate-determining step of the entire cathode reaction. Their models showed, when the former steps dominated, the total cell impedance would reduce to:

$$
Z=R_{\text {chem }} \sqrt{\frac{1}{1+j \omega t_{\text {chem }}}}
$$

When the gas phase diffusion was the limit step, the overall cathode reaction impedance was:

$$
Z=\frac{R_{\text {gas }}}{1-j \omega R_{\text {gas }} C_{\text {gas }}}
$$

$R_{\text {chem }}$ and $R_{\text {gas }}$ are characteristic resistances, $t_{\text {chem }}$ is a relaxation time of oxygen surface exchange and $C_{\text {gas }}$ is the effective capacitance with gas diffusion polarization. Their results showed that the ion conductivity of the cathode would considerably affect the electrode kinetics. To verify this simulated result, Adler et al ${ }^{143}$. compared the measured ac response of a symmetrical cell, which is composed of $\mathrm{La}_{0.6} \mathrm{Sr}_{0.4} \mathrm{Fe}_{0.8} \mathrm{Co}_{0.2} \mathrm{O}_{3-\delta}$ electrodes and $\mathrm{Ce}_{0.9} \mathrm{Gd}_{0.1} \mathrm{O}_{2-\mathrm{x}}$ electrolyte. As Figure 2.11 presents, the measured plots agreed well with the calculated results. Adler also proposed the concept of an active length $l_{\delta}$ which described the extension of the reaction zone beyond the three-phase boundary. The extension length was calculated with the chemical capacitance and reached to few microns. 


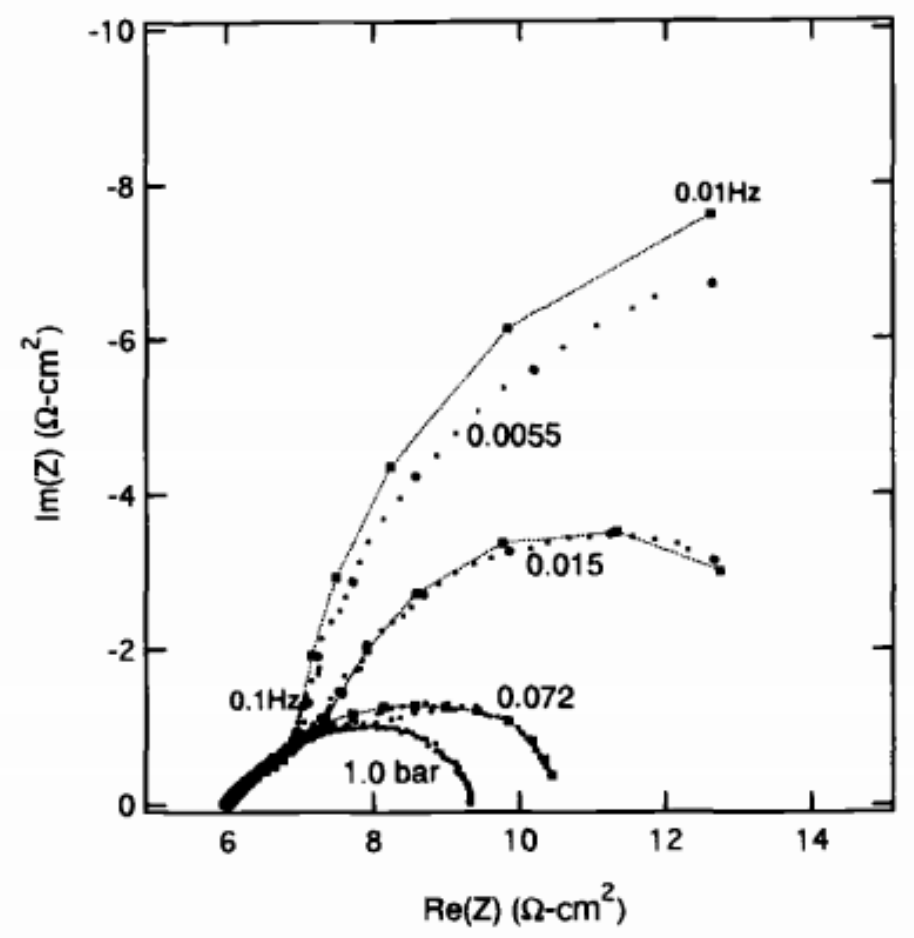

Figure 2.11 Plot of measured (circles) and calculated (squares) complex impedance of a symmetric cell in different $P_{\mathrm{O}_{2}}$ at $700^{\circ} \mathrm{C}$.

Adler's work offered valuable insight into the cathode reaction mechanism. However, researchers doubted whether it is reasonable to deem all the reduction reactions occurred at the electrode/gas interface ${ }^{144}$. Besides, if the oxygen is reduced to ions, the oxygen surface exchange should be defined as a charge transfer process. Adler responded to these criticisms in a subsequent paper ${ }^{145}$, where he argued that the oxygen reduction should only occur at the electrode/gas interface since matter cannot pass through a truly 3PB. He also clarified the charge transfer and non-charge-transfer definitions. "Charge-transfer" involves any charged species driven directly by electrochemical gradients and its rate always proportional to the current. On the other hand, "non-charge-transfer" involves neutral species or neutral combinations of species driven by chemical potential and its rate independent of the current. 
ORR was discussed based on symmetrical cells in this paper ${ }^{145}$. The author concluded that the ORR was retarded by the oxygen diffusion step and the diffusion process was independent of the electrochemical factors since molecular oxygen is neutral. The model ignored the effects of electron holes inside the mixed conductor. However, they would certainly affect the ORR. Furthermore, since the electrons and oxygen vacancies are chargeable, electrochemical conditions may control their diffusion process. Therefore, Adler's model may be more suitable for the starting period of the fuel cell operation.

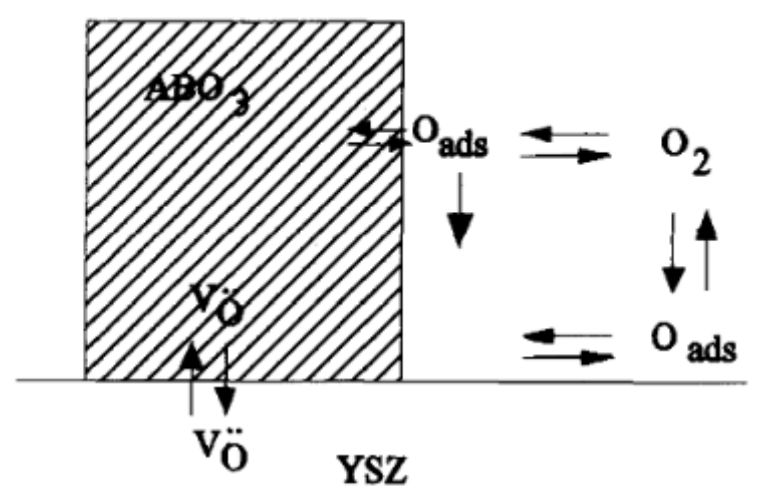

a)

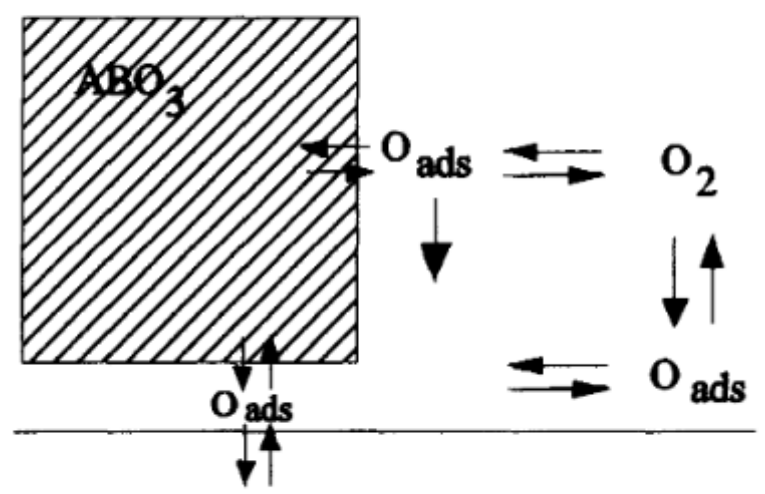

YSZ

b)

Figure 2.12 Sketch of Svensson's model considering chemical and electrochemical reactions.

Svensson et al. developed a physical model considering both the chemical and electrochemical reactions to illustrate the possible oxygen transport pathways on SOFC cathodes ${ }^{146-148}$. As 
shown in Figure 2.12, the first step of oxygen reduction is the adsorption and dissociation of neutral oxygen at the gas/cathode and gas/electrolyte interfaces. Then the absorbed oxygen atom will combine with the oxygen vacancy to form lattice oxygen. The two-step reactions can be written as:

$$
\begin{gathered}
\mathrm{O}_{2}(\mathrm{~g})+2 \mathrm{ads} \stackrel{\mathrm{k}_{\mathrm{ads}} \mathrm{k}_{\mathrm{des}}}{\longleftrightarrow} 2 \mathrm{O}_{\mathrm{ads}} \\
\mathrm{O}_{\mathrm{ads}}+\mathrm{V}_{\mathrm{O}} \stackrel{\mathrm{k}_{\mathrm{o}} \mathrm{k}_{\mathrm{r}}}{\longleftrightarrow} \mathrm{ads}+2 \mathrm{~h}+\mathrm{O}_{\mathrm{o}}^{\mathrm{x}}
\end{gathered}
$$

Svensson assumed that there could have intermediate oxygen species at the cathode/electrolyte interface $^{148}$. Those species would combine with either the vacancies of the cathode or the vacancies of the electrolyte. The reaction occurring at the gas/cathode surface was considered chemical since no charge transfer was involved, while the one occurring at the electrolyte surface was considered as an electrochemical process. Therefore, different from Adler's model, Svensson et al. introduced over-potential into their model to express the equilibrium deviation of the surface exchange reaction occurring at the electrolyte surface. According to Svensson's study, a limiting current was predicted at high over-potential due to depletion of oxygen at the cathode/electrolyte interface. Besides, they found there was a correlation between the limiting current and $P_{\mathrm{O}_{2}}\left(i \propto P_{\mathrm{O}_{2}}^{\mathrm{n}}\right)$. When the surface exchange process was the rate-limited step for the cathode reaction, the value of $n$ was between 0.58 and 0.74 . Smaller $n$ values $(0.26<n<0.56)$ were predicted for a slow adsorption process.

With Svensson's model, the influence of surface and bulk transport processes for SOFC cathode reaction can be described, but the contribution of each process cannot be quantitatively determined. To answer this question, Coffey et al ${ }^{149}$ proposed a continuum model which 
simultaneously considered both pathways, as shown in Figure 2.13. They considered that oxygen may transport through the triple-phase boundary (3PB) and the two-phase boundary (2PB) between the cathode and electrolyte. B-V equations for the surface over-potentials were taken as the boundary conditions in this model. Since the total voltage drop across the cathode-electrolyte interface is independent of the transport path chosen, the over-potential for the 3PB path can be deemed as the over-potential for the entire reaction. However, although Coffey treated the reactions occurring at $3 \mathrm{~PB}$ and $2 \mathrm{~PB}$ interfaces as electrochemically driven reactions, oxygen reduction occurring at the cathode surface was considered as a chemical reaction since there is no net change at the cathode.

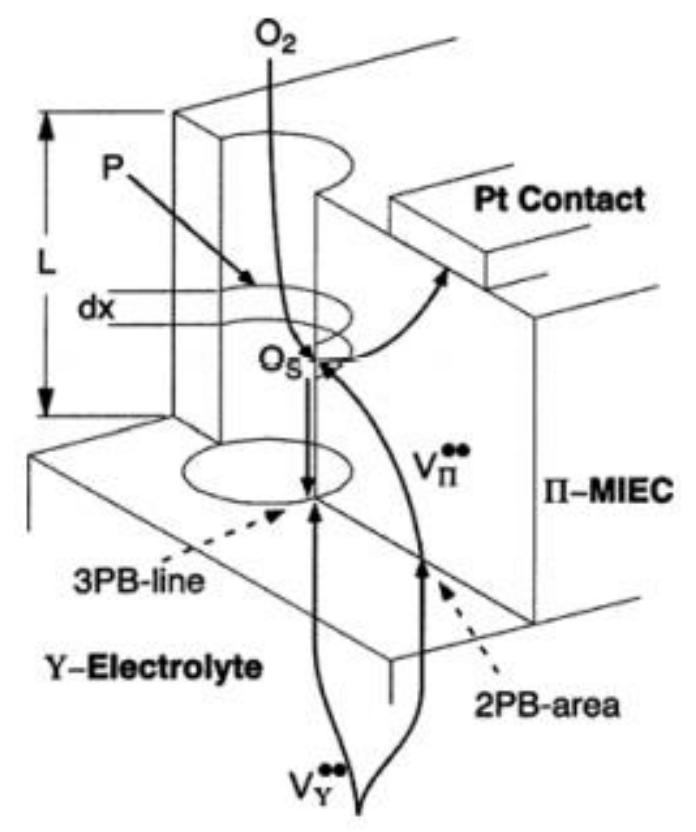

Figure 2.13 Cross-sectional scheme of the physical structure and chemical reactions occurring at porous SOFC cathode. 
In 2006, researchers from NASA build a SOFC model for stack controls and stability design ${ }^{150}$. The cathode elementary reactions were associated with oxygen adsorption, dissociation, diffusion, and electronation. The charge transfer pathways can be presented as below,

$$
\begin{gathered}
\mathrm{O}_{2}(\mathrm{~g})+2 \mathrm{~s} \stackrel{\mathrm{k}_{\mathrm{ads}, \mathrm{k}_{\mathrm{des}}}}{\longleftrightarrow} 2 \mathrm{O}_{\mathrm{ads}} \\
\mathrm{O}_{\mathrm{ads}}+\mathrm{V}_{\mathrm{O}}+2 \mathrm{e}^{-} \stackrel{\mathrm{k}_{\mathrm{lc}, \mathrm{k}_{-} \mathrm{lc}}}{\longleftrightarrow} \mathrm{s}+\mathrm{O}_{\mathrm{o}}^{\mathrm{x}}
\end{gathered}
$$

where $\mathrm{s}$ is the concentration of vacant surface sites. From the cathode reaction mechanisms stated above, researchers considered the oxygen reduction as a pure chemical process because they thought there was no net charge transfer and charged intermediate cannot stay at the interface. However, charged intermediates are possibly formed during the real oxygen reduction reaction. Furthermore, although there may be no net charge transfer at the interface many researchers hold that the reaction should be considered as an electrochemical process since its reactants and products are charged particles.

\subsubsection{Electrochemical Process}

Considering oxygen reduction is a reaction involving charged particles, many researchers hold that the cathode reaction of SOFC is an electrochemical process. It is accepted that the surface over-potential influences the entire cathode reaction. Besides, researchers argue that oxygen reduction is not completed in one step which means some intermediates exist during the reaction process. So, absorbed and charged intermediates will be affected by the surface over-potential. Liu and Winnick ${ }^{151}$ investigated the reactions occurring at MIEC/gas interface. They considered the oxygen reduction reaction involving several intermediates. Possible reaction processes are listed below: 


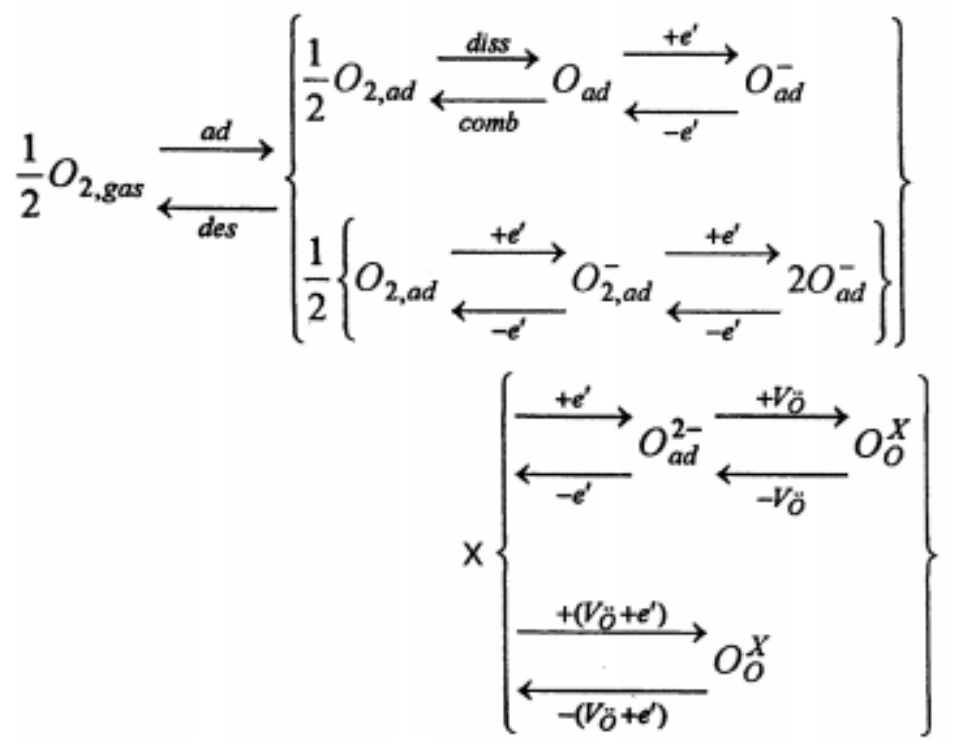

Figure 2.14 Possible oxygen reduction processes by Liu et al ${ }^{151}$.

Further, Liu et al. ${ }^{151}$ discussed the effects of the electrical state on the rate of interfacial reactions. Based on their analysis, the oxygen reduction rate depends critically on the electrical state of the MIEC/gas surface. Hence the reaction was considered electrochemical.

F. H. van Heuveln ${ }^{152}$ considered the existence of $\mathrm{O}^{-}, \mathrm{O}_{2}^{-}$during the oxygen reduction process, and assumed the three possible charge transfer pathways. $\mathrm{La}_{0.85} \mathrm{Sr}_{0.15} \mathrm{MnO}_{3}$ cathode was prepared by tape casting on a pre-sintered YSZ pellet. Electrochemical resistance and Tafel plots were measured to verify van Heuveln's model. The experimental results indicated that diffusion of $\mathrm{O}_{\mathrm{ads}}^{-}$species from the LSM surface to the $3 \mathrm{~PB}$ area will compete with charge-transfer at low over-potential. The diffusion limitation disappears at high cathodic over-potentials. On the other side, the model's simulation results also proved that the diffusion process is influenced mainly 
by the over-potential. However, in van Heuveln's model, the reduction occurring at the gas/cathode surface was ignored and the MIEC was treated similarly to the metal electrode.

Mitterdorfer ${ }^{153}$ also thought $\mathrm{O}^{-}, \mathrm{O}_{2}^{-}$may exist during the reduction process. He developed a physical model to explain how oxygen transport from the LSM cathode to the YSZ electrolyte. The reactions can be described as follow:

$$
\begin{gathered}
\mathrm{O}_{2}(\mathrm{~g})+\mathrm{e}^{-}+\mathrm{s} \leftrightarrow \mathrm{O}_{2}^{-}(\mathrm{ads}) \\
\mathrm{O}_{2}^{-}(\mathrm{ad})+\mathrm{e}^{-} \leftrightarrow 2 \mathrm{O}^{-}(\mathrm{ads}) \\
\mathrm{O}^{-}(\mathrm{ads})+\mathrm{e}^{-}+\mathrm{V}_{\mathrm{O}}^{*} \leftrightarrow \mathrm{O}_{\mathrm{O}}^{\mathrm{x}}+\mathrm{ad}(\mathrm{ads})
\end{gathered}
$$

In Mitterdorfer's model, LSM had been considered as a pure electronic conductor. The oxygen ions were assumed to be formed only at the $3 \mathrm{~PB}$ area.

Chan et al. developed a micro-model using an LSM electrode ${ }^{154}$. All possible polarizations which govern the complex interdependency among the transport phenomena, electrochemical reaction, and microstructure of the electrode and their combined effect on the cathode overpotential under different operating conditions had been considered in this model. They claimed that when the applied oxygen partial pressure was lower than $0.1 \mathrm{~atm}$, a third arc can be seen in the low-frequency band of impedance spectra which is due to gas phase diffusion.

Chan applied the reaction steps of van Heuveln's model to develop their model. The difference is that Chan et al. established a correlation between the microstructure and the cathode performance. According to the simulation results, a larger cathode crystallite size requires a thicker cathode for cathode over-potential. Furthermore, the current density and oxygen partial 
pressure was found to not affect the optimal electrode thickness value. However, current density and oxygen partial pressure will affect the optimal crystallite size since the cathode porosity and tortuosity change with applied current or gas atmosphere change.

Bilge Yildiz set up a 2-D physical model that includes the effect of both surface and bulk pathways under different operating conditions and electrode configurations ${ }^{155}$. The possible surface and bulk pathways were shown below, and AC impedance spectra of LSM electrode were applied to verify Yildiz's model:

To prove that the intermediates are likely to exist during the oxygen reduction process, Liu et al. investigated the oxygen reduction process on a silver electrode surface using the first-principles calculations based on the density functional theory and pseudo-potential method ${ }^{156}$. The calculation results suggest that the oxygen reduction reaction on silver cathode can be described as:

$$
\mathrm{O}_{2}(\mathrm{~g})(+\mathrm{e}) \rightarrow \mathrm{O}_{2}^{-}(+\mathrm{e}) \rightarrow\left(\mathrm{O}_{2}^{2-}+2 \mathrm{e}^{-}\right) \rightarrow 2 \mathrm{O}^{2-}
$$

Since a metal electrode has been studied in this work, the calculation results show that the oxygen reduction and the incorporation of the dissociated $\mathrm{O}$ ions in the oxide electrolyte prefer the 3PB region as it should be. For MIEC electrodes, because oxygen vacancy may exist inside the material, the reduction process will not only occur near the 3PB region. However, although this work offers some evidence for the intermediates, it's not so convincing that they will appear in perovskite MIEC. Therefore, researchers still examined new ways to verify the existence of the intermediates and to confirm the intermediates appearing during the reduction process. 
Since researchers considered the cathode reaction as an electrochemical process, surface overpotential needs to be introduced into their simulations. To make a clear idea on this issue, first, we need to know what the over-potential is and how it is generated. Over-potential refers to the potential difference between a half-reaction's thermodynamically determined reduction equilibrium potential and the potential at which the redox event is experimentally observed. For an aqueous system, the over-potential is formed at the electrode/electrolyte interface. For the SOFC cathode, the over-potential exists not only at the electrode/electrolyte interface but also at the gas/electrode interface and the TPB area. And there are three different parts for the overpotential: overpotential caused by the material's resistance, over-potential caused by the surface exchange process, and over-potential caused by the oxygen ion diffusion.

J. Fleig discussed MIEC surface over-potential as caused by surface charge transfer process ${ }^{157}$. Figure 2.15 depicts the over-potential difference between a liquid electrolyte system and the SOFC cathode. For a metal electrode in an aqueous electrochemical system, electron and ion transfer only occur at the electrode/electrolyte interface so the over-potential can only be formed at the electrode/electrolyte interface. However, for mixed conducting electrodes, electron and ion transfer also occur at the electrode/gas interface. Hence it's necessary to introduce the term of surface over-potential change $\Delta \chi$ into the simulation. Fleig discussed the application of $\Delta \chi$ under electron transfer step control and ion transfer step control conditions. He also studied the relationship between MIEC surface over-potential $(\Delta \chi)$ and electrode/electrolyte interface overpotential ( $)$. 


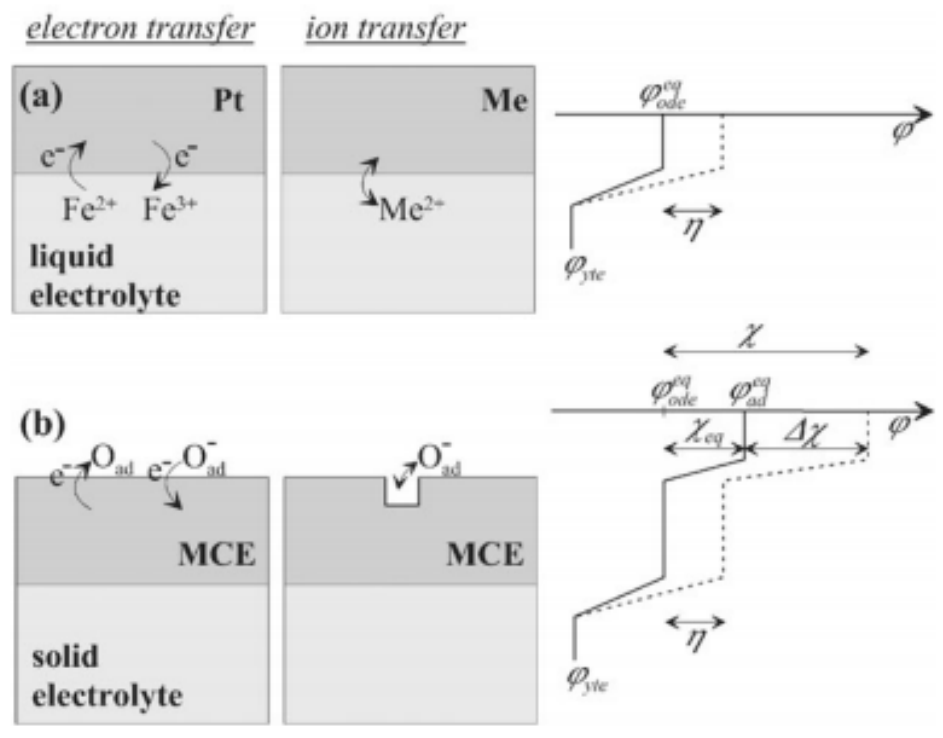

Figure 2.15 Schemes of electron and ion transfer reactions at (a) metal/liquid electrolyte and (b) MIEC/solid electrolyte interfaces.

In later work, the equivalent circuit based on the understanding of the oxygen reduction pathways was presented, as Figure 2.16 shows ${ }^{158,159} . \quad \mathrm{La}_{0.6} \mathrm{Sr}_{0.4} \mathrm{Co}_{0.8} \mathrm{Fe}_{0.2} \mathrm{O}_{3-\delta}$, $\mathrm{Ba}_{0.5} \mathrm{Sr}_{0.5} \mathrm{Co}_{0.8} \mathrm{Fe}_{0.2} \mathrm{O}_{3-\delta}$ and $\mathrm{Sm}_{0.5} \mathrm{Sr}_{0.5} \mathrm{CoO}_{3-\delta}$ thin films were applied to study the oxygen reduction. Thin films and patterned electrodes are usually used for investigating over-potential effects because it is possible to precisely control the geometry related to the triple-phase boundary and bulk reaction pathways. Based on the ac and dc resistance measurements, Fleig claimed that the capacitance in Figure 2.15 was a constant phase element $\left(Q^{-1}(i \omega)^{-n}\right)$ with exponents close to one. And they found for the investigated materials, the oxygen exchange reaction on the MIEC surface limited the kinetics of the overall oxygen reduction reaction. On the other hand, according to the experimental results, $\mathrm{Ba}_{0.5} \mathrm{Sr}_{0.5} \mathrm{Co}_{0.8} \mathrm{Fe}_{0.2} \mathrm{O}_{3-\delta}$ exhibited the lowest surface-related polarization resistance compared to the other two materials. 
(a)

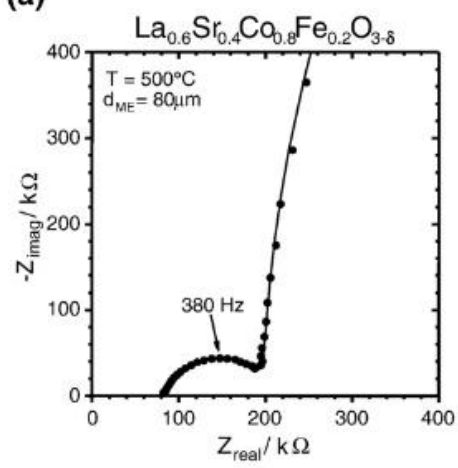

(d) (b)

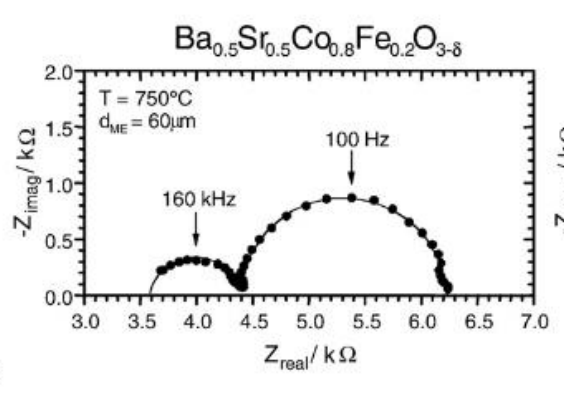

(c)

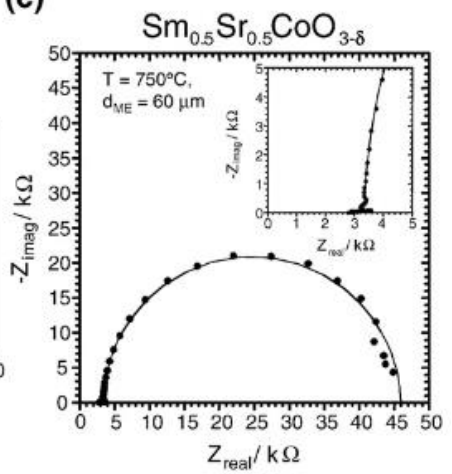

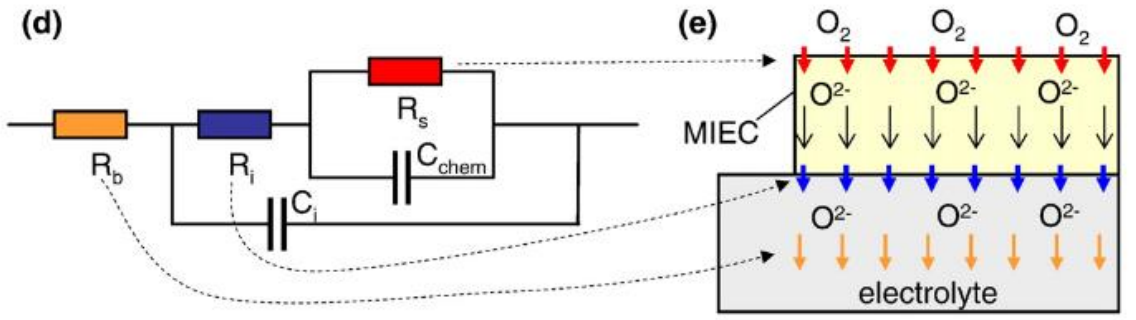

Figure 2.16 Sketch of Nyquist plots of MIEC electrodes, equivalent circuit, and the ORR process.

Liu developed a 2-D model based on Fleig's theory of MIEC surface over-potential ${ }^{160}$. Figure 2.17 depicts their model geometry. As Figure $2.17 \mathrm{~b}$ shows, the dashed-line box is the 2-D investigated region. The gas exposure surface reactions were assumed as below:

$$
\begin{gathered}
\frac{1}{2} \mathrm{O}_{2}+\mathrm{s} \rightarrow \mathrm{O}_{\mathrm{ads}}^{\prime}+\mathrm{h}^{\cdot} \\
\mathrm{O}_{\mathrm{ads}}^{\prime}+\mathrm{V}_{\mathrm{O}}^{\prime} \rightarrow \mathrm{O}_{\mathrm{O}}^{\mathrm{x}}+\mathrm{h}^{\cdot}+\mathrm{s}
\end{gathered}
$$

The reaction rate had been discussed in their previous work ${ }^{161}$. The simulation results show that under low over-potential the ionic transportation will be the rate-limiting step which means low over-potential $(50 \mathrm{mV})$ does not affect oxygen reduction. Under high over-potential $(750 \mathrm{mV})$, the sheet resistance will control the reaction rate which means that the surface exchange step will be the rate-limiting step. 


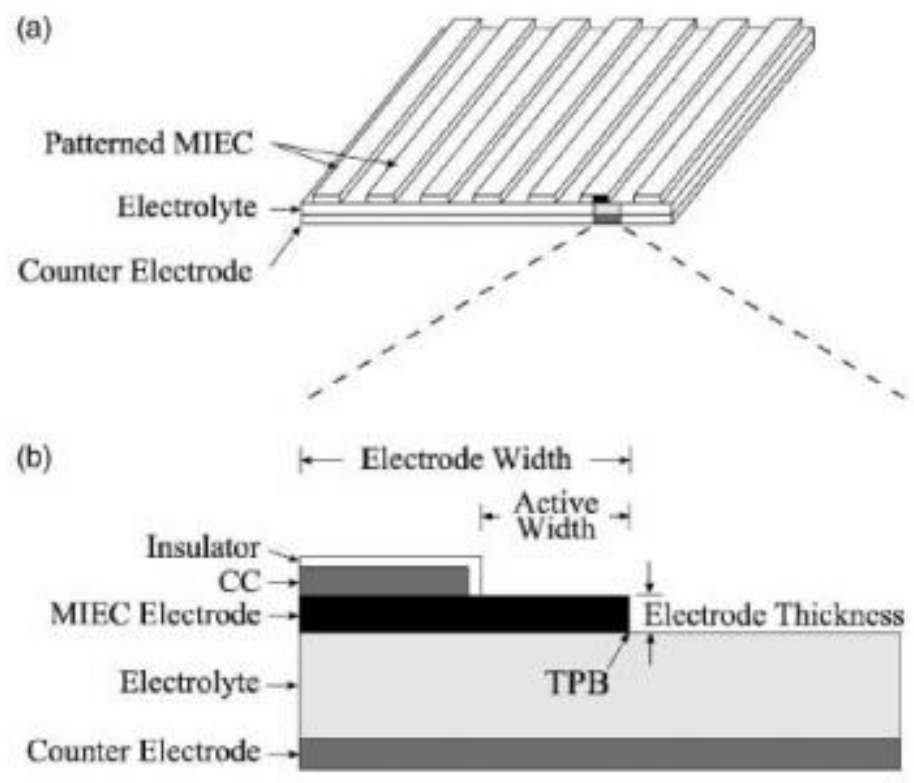

Figure 2.17 Scheme of the 2-D model (a) patterned electrode array (b) symmetric 2-D cross-sectional model domain.

Gong et al developed a 1-D continuum model based on LSM-type cathode by incorporating multi-step charge transfer into the bi-pathway kinetics, as shown in Figure $2.7^{127}$. The surface $3 \mathrm{~PB}$ pathway and the bulk $2 \mathrm{~PB}$ pathway model were established to provide a numerical treatment method to analyze multi-step charge transfer. The finite control-volume method is used to simulate the pathway kinetic competition, and a parametric study is performed with different equilibrium concentrations of surface oxygen ion and bulk oxygen vacancy. The $I-V$ profiles show the kinetic transition from $3 \mathrm{~PB}$-control to $2 \mathrm{~PB}$-control at overpotentials from $-0.2 \mathrm{~V}$ to $-0.4 \mathrm{~V}$, and the active reaction zone in concentration profiles expands from $3 \mathrm{~PB} / 2 \mathrm{~PB}$ interfaces by $2-4 \mu \mathrm{m}$. The exchange and local 3PB currents recognized from simulation indicate the limit of surface oxygen diffusion as the mechanistic process for pathway transition. The surface reaction elementary steps S1-S4, and the bulk reaction elementary steps of B3 and B4 are listed in Table 2.2. The bulk 2PB reaction shares the $\mathrm{S} 1$ and $\mathrm{S} 2$ two steps with the surface 3PB reaction. 
Table 2.2 Electrode processes assigned to each elementary step in surface and bulk pathways ${ }^{127}$.

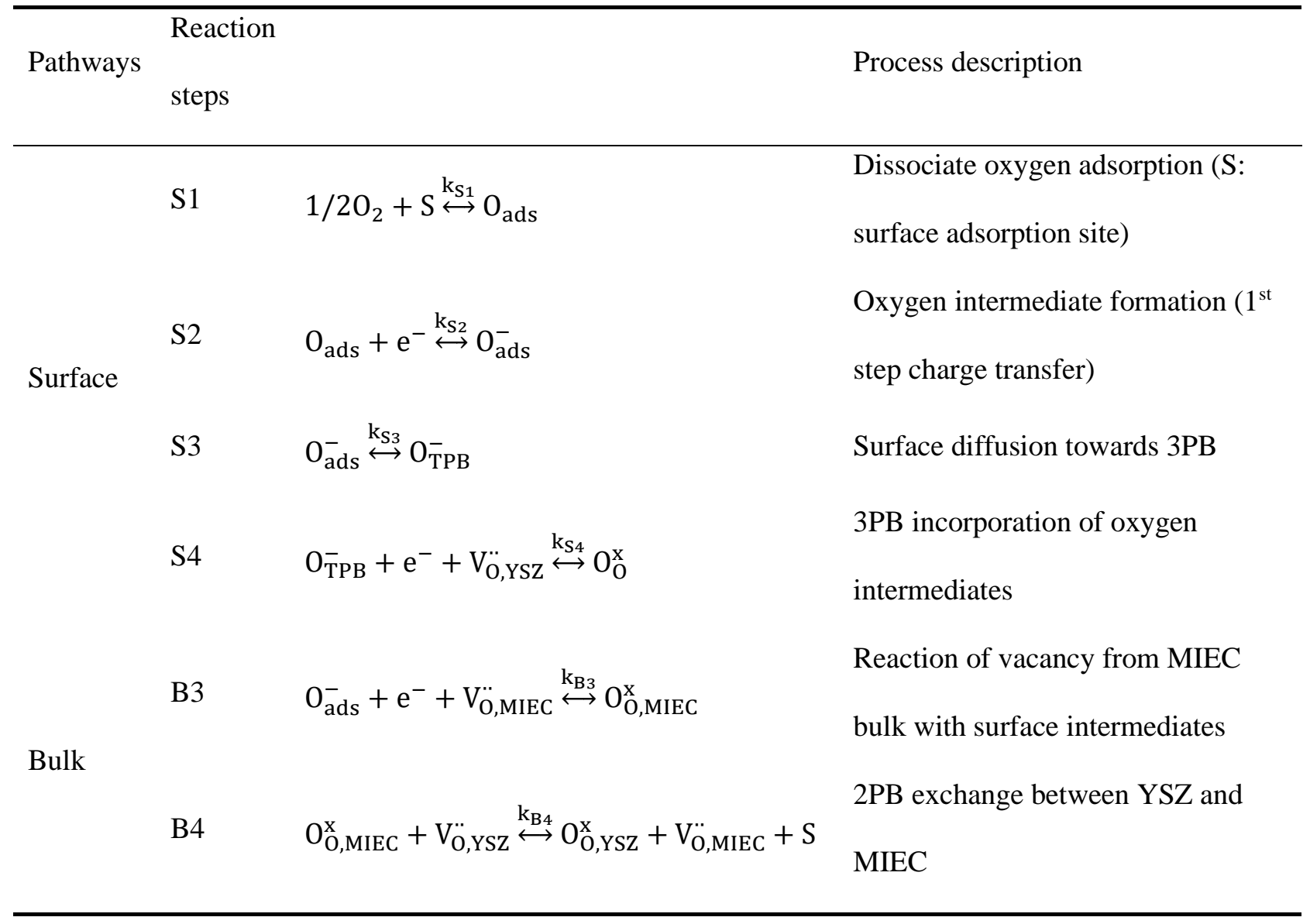

The literature reviewed in this part has evidenced that the oxygen reduction mechanism in the SOFC cathode is complex. Although various experimental methods have been utilized to verify model results, differences of opinion still exist among researchers. Several main debated issues about SOFC cathode reaction mechanisms are summarized as below: (1) Reaction intermediates: Different elementary reactions were proposed by researchers for the same entire oxygen reduction reaction. Adsorbed oxygen atoms, $\mathrm{O}^{-}$and $\mathrm{O}_{2}^{-}$all possibly appear via one or more steps. The surface over-potential needs to be considered when there are chargeable intermediates during the reduction process. However, based on the presently investigated literature, it is still difficult to determine which intermediates exist in the reaction. (2) Reaction region: Generally, the $3 \mathrm{~PB}$ region was supposed to be the main reaction region. However, since the cathode 
material is a mixed ionic and electronic conductor, oxygen reduction also may occur at the gas/cathode surface. Therefore, two charge transfer pathways will co-exist and may compete under different operation conditions. (3) Rate-limited steps: The global oxygen reduction can be divided into several elementary reactions according to the understandings of the oxygen reduction mechanism. Besides the reduction reactions, oxygen diffusion is the other step for the cathode reaction process. Rate-limited step determination under different conditions is important for optimizing the cathode and improving the cell efficiency. (4) Geometry factors: Cathode geometrical factors such as porosity, tortuosity, and TPB length are important for SOFC cathode modeling. They will affect the gas diffusion process and cell performance. Because the shape and the distribution of the pores inside SOFCs cathode are random, it's difficult to get an accurate simulation result. Some researchers applied a factor parameter to minimize the errors caused by ignoring the geometry effects. Most researchers used a computer to build up a random microstructure with some input parameters. An effective way is to get part of the microstructure information from the experimental method first, then use a computer program to simulate the entire cathode reaction process. 


\section{Research Objectives}

Develop a double perovskite cathode with high performance for SOFC.

Evaluate the structure of the developed materials in both air and nitrogen, chemical bonding, electrical conductivity, and the electrochemical performance of symmetrical cells and threeelectrode cells.

Investigate the crystal structure of $\mathrm{PBCO}$ material with two pristine phases (tetragonal and orthorhombic) with Neutron Powder Diffraction (NPD) and Transmission Electron Microscopy (TEM).

Measure the oxygen non-stoichiometry values within the intermediate-temperature SOFC range. Study and compare the thermodynamic property of PBCO based on the relation between oxygen non-stoichiometry $\delta$, temperature $T$, and oxygen partial pressure $P_{\mathrm{O}_{2}}$.

Analyze the thermodynamic property based on the crystal structure investigation.

Characterize and compare the oxygen bulk diffusion performance of PBCO cathodes.

Investigate the influence of phase structure evolution and thermodynamic property on the oxygen transport performance in PBCO cathodes. Study the oxygen vacancy formation and migration energy of tetragonal and orthorhombic PBCO.

Research the oxygen reduction reaction (ORR) on PBCO cathodes and determine the ratedetermining step. 


\section{Experimental Methods}

\subsection{Synthesis of Materials}

The PBCO powders were synthesized through EDTA-citrate complex sol-gel process ${ }^{162}$. A stoichiometric amount of raw materials $\operatorname{Pr}\left(\mathrm{NO}_{3}\right)_{3} \cdot 6 \mathrm{H}_{2} \mathrm{O}, \mathrm{Ba}\left(\mathrm{NO}_{3}\right)_{2} \cdot 6 \mathrm{H}_{2} \mathrm{O}, \mathrm{Co}\left(\mathrm{NO}_{3}\right)_{2} \cdot 6 \mathrm{H}_{2} \mathrm{O}$ (from Alfa Aesar or Acros Organics), were dissolved in distilled water in one beaker (solution 1). In another beaker, the mixture of ethylenediaminetetraacetic acid (EDTA) (Alfa Aesar) and a suitable amount of diluted ammonia (Alfa Aesar) were heated and stirred until a clear solution was obtained (solution 2). Solution 2 was mixed into solution 1 and citric acid was (Alfa Aesar) added. The molar ratio of metal ions: citric acid: EDTA was 1:1.25:1. The purple solution was adjusted to $\mathrm{pH}=6$ with diluted ammonia and held on a hot plate at about $110^{\circ} \mathrm{C}$ for several hours with stirring until gelation formed. The gel was heated at $220{ }^{\circ} \mathrm{C}$ overnight to drive off the liquid and form porous black ash.

The black ash was fired at about $450{ }^{\circ} \mathrm{C}$ with intermittent stirring until all organic residues are eliminated. The black precursor was obtained after this low-temperature treatment. This is the most important step during the synthesis process. As abundant EDTA was added into the solution, the organics in the precursor must be eliminated thoroughly before a phase formation, especially for a big batch. Otherwise, some carbonized organics will stay in the precursor and form amorphous structures with a metal oxide or change the local condition for the phase formation.

Similar synthesis routines with a thorough organic residues elimination process were applied to all materials (PBCO, NBCO, YBCO, $\mathrm{LBCO}$ ) in this work. 


\subsection{Fabrication of Cells}

\subsubsection{LnBCO Symmetrical Cells}

LnBCO symmetrical cells with GDC electrolytes were fabricated by the screen-printing method. GDC pellets (diameter is $16 \mathrm{~mm}$ ) were sintered at $1400{ }^{\circ} \mathrm{C}$ in the air for $4 \mathrm{~h}$. The diameter of the sintered electrolyte discs was about $13 \mathrm{~mm}$. To make cathode slurry, the as-synthesized tetragonal and the pristine orthorhombic LnBCO powders were ground in an ink vehicle (Fuel Cell Materials Co.) with the weight ratio of cathode powders: vehicle 10:14. Cathode slurry was print coated onto both sides of the electrolyte symmetrically. The number of print-coating cycles depended on the designed electrode thickness. Typically, a single print-coating resulted in $10 \mu \mathrm{m}$ in thickness after sintering. The effect electrode area was $0.7 \mathrm{~cm}^{2}$ on each side. The as-fabricated symmetrical cells were sintered at $1000{ }^{\circ} \mathrm{C}$ for $2 \mathrm{~h}$ in the air to achieve a good interface connection and a porous electrode. The silver paste was brushed on both sides of electrodes as the current collector, followed by heating at $650{ }^{\circ} \mathrm{C}$ for $0.5 \mathrm{~h}$.

\subsubsection{LnBCO Three Electrodes Cells}

LnBCO three-electrodes cells with GDC electrolytes were fabricated by the screen-printing method. GDC pellets (diameter is $16 \mathrm{~mm}$ ) were sintered at $1400{ }^{\circ} \mathrm{C}$ in the air for $4 \mathrm{~h}$. The diameter of the sintered electrolyte discs was about $13 \mathrm{~mm}$. To make working electrode slurry, the as-synthesized tetragonal and the pristine orthorhombic $\mathrm{LnBCO}$ powders were ground in an ink vehicle (Fuel Cell Materials Co.) with the weight ratio of cathode powders: vehicle 10:14. Working electrode slurry was print coated onto one side of the electrolyte. The effect electrode area was $0.7 \mathrm{~cm}^{2}$. The cells were sintered at $1000{ }^{\circ} \mathrm{C}$ for $2 \mathrm{~h}$. The counter electrode silver paste (Fuel Cell Materials Co.) was symmetrically printed onto the opposite side of the cathode. Silver 
wire (Surepure Chemetals) was used as a reference electrode and embedded into the middle circle of the electrolyte cross-section with silver paste. The cells were then sintered at $650{ }^{\circ} \mathrm{C}$ for $0.5 \mathrm{~h}$ to get a good connection with silver paste.

\subsection{Materials Characterization}

\subsubsection{XRD, SEM, and EDS}

The powder phase purity and mixture powders compatibility were examined through X-ray diffraction (XRD, PANalytical X'pert PRO, and Burker AXS, $\mathrm{Cu} \mathrm{K} \alpha$ radiation, model number PW 3040 Pro, National Energy Technology Laboratory). The instrument power was set to $45 \mathrm{kV}$ and $40 \mathrm{~mA}$. The normal XRD patterns were collected with a $0.026 \% \mathrm{~s}$ scan rate and a $120 \mathrm{~s}$ step time. The detailed scans were collected using a $0.013 \%$ scan rate and 400 s step time for refinement use. Commercial software Jade 6.5 and X'pert Highscore Plus were used to analyze XRD spectra.

In-situ XRD was performed to evaluate the change in the crystal structure of the LnBCO materials during heating under conditions similar to those used for calcination. The sample was placed in an 8mm alumina sample holder in an Anton Parr HTK 1200N high-temperature oven chamber. A TCU-1000N control unit, with a Eurotherm 2604 controller was used to control temperature, the $\mathrm{PBCO}$ materials were heated from room temperature to $700{ }^{\circ} \mathrm{C}$ with $500{ }^{\circ} \mathrm{C}, 550$ ${ }^{\circ} \mathrm{C}, 600{ }^{\circ} \mathrm{C}, 650{ }^{\circ} \mathrm{C}$, and $700{ }^{\circ} \mathrm{C}$ as the target temperatures. Instrument settings were similar to those described earlier, as were the scan parameters $0.013 \%$ s scan rate and a $400 \mathrm{~s}$ step time. The heating profile was similar to the calcination used for powder preparation. A $5{ }^{\circ} \mathrm{C} / \mathrm{min}$ ramp rate was used to heat from ambient to $273{ }^{\circ} \mathrm{C}$ where the first scan was performed. The materials were 
then heated incrementally by $5{ }^{\circ} \mathrm{C} / \mathrm{min}$ to $500{ }^{\circ} \mathrm{C}$ to $700{ }^{\circ} \mathrm{C}$, with 6 isothermal scans performed at each temperature.

The Rietveld refinement of the PBCO crystal structure was performed with software TOPAS Academic v6. The instrument profile and detector zero were obtained from the refinement of an external silicon standard. The background was modeled using a fifth-order polynomial function, the peak shape was modeled using the modified Thompson-Cox-Hastings pseudo-Voigt function 163. The peak broadening was modeled by the crystallite size function. The initial structural model was from the Crystallography Open Database (COD), \#00-053-0131 and \#04-013-5730. The site occupancies of the elements $(\mathrm{Pr}, \mathrm{Ba}, \mathrm{Co}, \mathrm{O})$ were from the nominal chemical composition.

Scanning Electron Microscopy (SEM, JEOL JSM-7600F, Hitachi S-4700) and Energy Dispersive X-Ray Spectroscopy (EDS) (National Energy Technology Laboratory) were utilized separately or together to observe the microstructure and the distribution of the elements of the powders and cells. Data collection was operated with a working distance of $12 \mathrm{~mm}$, voltage 15 $\mathrm{kV}$, current $10 \mu \mathrm{A}$. The sample was coated with an $\mathrm{Au}$ conductive layer in a sputter chamber (Denton Desk V Sputter and Carbon Coater) before SEM characterization.

\subsubsection{Transmission Electron Microscopy Sample Preparation and Characterization}

The mixture of the synthesized PBCO powder (wt.\% 61.41\%), XYLENE (wt. \%: 15.55\%, Alfa Aesar), Ethanol (wt.\%: 15.55\%, Alfa Aesar), and Fish oil (wt. \%: 1.3\%, Alfa Aesar) was heated and stirred in a $50 \mathrm{~mL}$ beaker until a clear solution 1 was obtained. Butyl Benzyl Phthalate (wt. \%: 1.55\%, TCW), Polyvinyl Butyral (wt. \%: 3.08\%, TCW), Polyethylene glycol (wt.\%: $1.55 \%$, Alfa Aesar) were added to the solution 1, then heated and stirred until a clear solution 2 
was obtained. The $5 \mathrm{~mL}$ dropper was used to drop solution 2 on a glass plate. A tape with 20micron thickness was pasted on the glass plate to avoid trickling. The glass plate was then put into a fume hood and wait until the plastic thin film dry. The thin film was peeled off the plate and then sintered at $1100{ }^{\circ} \mathrm{C}$ for 2 hours in air and nitrogen, respectively. The wafers were then used for TEM sample preparation and characterization. The pretreatment on the PBCO powders with the above process can avoid the drawback of TEM characterization on powders and save the pellets milling and the thinning time with the Focused Ion Beam (FIB).

The wafers were thinned with FEI Plasma Dual Beam FIB and characterized with JEOL JEM2000EX TEM (Materials CharaTECrization Facility, Carnegie Mellon University). The data and morphology were analyzed with Gatan Microscopy Suite Software.

\subsubsection{Neutron Powder Diffraction and Rietveld Refinement at Oak Ridge National Laboratory}

Neutron diffraction can be utilized to gain similar information about the crystal structure as $\mathrm{X}$ ray diffraction with a few key differences in the measurement and the information gained about each atom. It utilizes neutrons rather than X-rays as the scanning beam. The neutron passes through the electron cloud (which reflects X-rays) without interaction and instead interacts directly with the atom's nucleus. Also, rather than measuring the scattering angle, neutron diffraction is measured using neutron time-of-flight scattering, where the initial neutron beam has a fixed initial position and velocity. This beam is broken into individual pulses, allowing the measurement of the position of deflected neutrons as well as the time after the pulse when they are detected. For a time-of-flight diffractometer, the time-of-flight can be linked to the diffraction through a combination of Bragg's law (Equation 4-1) and the de Broglie relation 
(Equation 4-2). This information can be utilized to determine the momentum and energies of the deflected neutrons and thereby provide accurate information about the crystal structure of the sample.

$$
\begin{array}{r}
l=2 d_{h k l} \sin Q \\
\frac{h}{l}=m_{n} \frac{L}{t}
\end{array}
$$

For the neutron diffraction data, the lattice parameter, oxygen fractional occupancy, isotropic atomic displacement parameters for each atom, and profile parameters $\left(\gamma_{1}, \sigma_{1}\right.$, and $\left.\sigma_{2}\right)$ were all refined for each phase individually. Atomic displacement parameters were typically refined for each crystallographic site, as for a disordered structure it is impossible to distinguish between elements located on a singular site. The DIFA and absorption parameters were constrained to be the same for all of the data sets for a given sample, as these should be constant with temperature. The scale factor was held constant when no change in phase was expected.

Each Rietveld refinement starts with default values with only instrument parameters, crystal symmetry, and atomic positions manually entered. From there, each refinement refines all parameters independently of all other refinements. The reported values and trends in oxygen stoichiometry (decreasing with increasing temperature and decreasing $P_{\mathrm{O}_{2}}$ ) and anisotropic or isotropic unclear density (increasing with increasing temperature and decreasing occupancy) are only compared at the end of all refinements and serve as a check on the relative accuracy of the refinements. Additional checks for accuracy include similar occupancy determination for the same, or very similar, materials by different research teams utilizing different diffractometers and sample environment systems ${ }^{164-166}$, and the correlation between structure controlled and diffraction determined oxygen stoichiometry. 
The powder Neutron Scattering diffraction pattern of the PBCO samples sintered in air and nitrogen $\left(1000^{\circ} \mathrm{C}\right.$ in the air for $12 \mathrm{~h}$, and $1000{ }^{\circ} \mathrm{C}$ in nitrogen for $\left.12 \mathrm{~h}\right)$ were collected at the HB2A beamline, High Flux Isotope Reactor (FLIR), Oak Ridge National Laboratory, Oak Ridge, TN, USA ${ }^{167}$. PBCO powders were suspended in a quartz basket $(1.0 \mathrm{~cm}, 4 \mathrm{~cm}$ long) inside a silica quartz tube. Metered $\mathrm{O}_{2} / \mathrm{N}_{2}$ gas mixtures fed to the quartz tube set the sample environment $P_{\mathrm{O}_{2}}$. For both the two kinds of PBCO samples, the tests were conducted at $P_{\mathrm{O}_{2}}=0.1,0.01,0.21 \mathrm{~atm}$, and in nitrogen at $500{ }^{\circ} \mathrm{C}, 550{ }^{\circ} \mathrm{C}, 600{ }^{\circ} \mathrm{C}, 650{ }^{\circ} \mathrm{C}, 700{ }^{\circ} \mathrm{C}$. An initial diffraction pattern was collected for $\mathrm{PBCO}$ at $25^{\circ} \mathrm{C}$ in nitrogen and air. Diffraction patterns were collected in the d-spacing range of $0.45-3.6 \AA$. Several short-duration 20 min patterns were collected upon changing the sample environment. Longer collection times (up to 2h) were collected once no change in primary diffraction peak position between short scans was observed. The measure of equilibration was typically achieved within $1 \mathrm{~h}$. Profile fitting was done using a TOF profile function which is a variation on the standard back-to-back exponential profile function. The lattice parameters, fractional occupancy of all oxygen sites, anisotropic thermal parameters, positions of all sites, and a combined scale factor were refined for each pattern.

Rietveld refinement was carried out using the software TOPAS Academic v6. The background was modeled using a ninth-order polynomial function. The wavelength, instrument profile, and detector zero were obtained from the refinement of a silicon standard (NIST SRM 640f). Userdefined empirical functions considering both Gaussian and Lorentzian contributions were used to fit the instrumental profile. The peak broadening of the phases was modeled by the crystallite size function. The initial structure models were from the Crystallography Open Database (COD), $\# 00-053-0131$ and \#04-013-5730. For the tetragonal PBCO phase, the site occupancies of $\mathrm{Pr}, \mathrm{Ba}$, $\mathrm{Co}$, and $\mathrm{O}$ were refined. Once good refinement was achieved, as indicated by low values of the 
weighted-profile R-value $\left(R_{\mathrm{wp}}\right)$ and goodness of fit $\left(\chi^{2}\right)^{168}$, the percentage of the involved crystalline phases were calculated by ${ }^{169}$,

$$
W_{p}=(S Z M V)_{p} / \sum_{i}(S Z M V)_{i}
$$

Where $W_{p}$ is the relative weight percentage of phase $p, S$ the scale factor, $Z$ the number of formula units per unit cell, $M$ the molecular weight of the formula unit, and $V$ the volume of the unit cell. $i$ represents each phase in the mixture.

\subsubsection{Iodometric Titration}

The pristine oxygen non-stoichiometry $\delta$ values in formula $\mathrm{PrBaCo}_{2} \mathrm{O}_{6-\delta}$ were determined by iodometric titration ${ }^{170} .20 \mathrm{mg}$ of samples were dissolved in $1 \mathrm{M} \mathrm{HCl}$ solution containing $\mathrm{KI}$, resulting in the reduction of $\mathrm{Co}^{3+}$ and $\mathrm{Co}^{4+}$ to $\mathrm{Co}^{2+}$ ions and the formation of a stoichiometric amount of iodine in the solution. Iodine was titrated with $\mathrm{Na}_{2} \mathrm{~S}_{2} \mathrm{O}_{3}$ solution using starch as an indicator. Starch was added just before the end-point to ensure accuracy. Oxygen-deficient values $\delta$ were calculated using the equations $4-4$ and 4-5. The calculated $\delta$ values are 0.32 and 0.5 which correspond to tetragonal and orthorhombic PBCO.

$$
\begin{aligned}
& \mathrm{Co}^{(3+x)}+(3+\mathrm{x}) \mathrm{I}^{-}=\mathrm{CoI}_{2}+\frac{1+\mathrm{x}}{2} \mathrm{I}_{2} \\
& \mathrm{I}_{2}+2 \mathrm{Na}_{2} \mathrm{~S}_{2} \mathrm{O}_{3}=2 \mathrm{NaI}+\mathrm{Na}_{2} \mathrm{~S}_{4} \mathrm{O}_{6}
\end{aligned}
$$

\subsubsection{Thermal Gravimetric Analysis (TGA)/Differential Thermal Analysis (DTA)}

A TA Instrument SDT Q600 unit was used for simultaneous DTA-TGA analysis of the PBCO powders. $12.5 \mathrm{mg}$ of sample was placed into a sapphire crucible and heated by $5^{\circ} \mathrm{C} / \mathrm{min}$ from room temperature to $700{ }^{\circ} \mathrm{C} .50 \mathrm{sccm}$ air purge was flown over the sample and blank reference 
crucible. The instrument provided weight loss as well as heat flow as a function of time and temperature. An online mass spectrometer was used to record concentrations of $\mathrm{H}_{2}, \mathrm{O}_{2}, \mathrm{~N}_{2}, \mathrm{CO}$, $\mathrm{CO}_{2}$, and $\mathrm{CH}_{4}$. To be concrete, the fresh samples for both phases have been going through an isothermal TGA curve at $500{ }^{\circ} \mathrm{C}, 550{ }^{\circ} \mathrm{C}, 600{ }^{\circ} \mathrm{C}, 650{ }^{\circ} \mathrm{C}$, and $700{ }^{\circ} \mathrm{C}$ in the air until the weight reached steady. Besides, the same heating process was carried out in $10 \%, 4 \%$, and $1 \%$ air. Oxygen non-stoichiometry change $(\Delta \delta)$ when heated to a certain temperature in a given oxygen content atmosphere was calculated from formula 4-6. $\mathrm{W}_{\mathrm{i}}$ and $\mathrm{W}_{\mathrm{f}}$ are initial and final sample weights. $\mathrm{W}_{\mathrm{Mi}}$ represents the initial molar weight. Together with $\delta$ values known at room temperature, oxygen deficiency amount can be determined.

$$
\Delta \delta=\frac{\left(\mathrm{W}_{\mathrm{i}}-\mathrm{W}_{\mathrm{f}}\right) \times \mathrm{W}_{\mathrm{Mi}}}{16 \mathrm{~W}_{\mathrm{i}}}
$$

\subsubsection{BET Surface Area}

Surface area measurements were performed using a Micromeritics ASAP 2020 instrument. 0.48 g sample was added to the sample tube with the filler rod placed in to minimize volume. The sample was heated to $90{ }^{\circ} \mathrm{C}$ with $10^{\circ} \mathrm{C} / \mathrm{min}$ ramp rate and hold for the pressure evacuated down to $10 \mu \mathrm{mHg}$. The sample was then heated to $300{ }^{\circ} \mathrm{C}$ and hold for $4 \mathrm{~h}$ to degas any absorbed contaminants. BET measurements were conducted using nitrogen adsorption at 77K. A 5-point, low surface area measurement was used as the samples were anticipated to have surface area $<5$ $\mathrm{m}^{2} / \mathrm{g}$. The range of $\mathrm{P} / \mathrm{P}_{0}$ ratio used for these experiments was $0.07-0.2$.

\subsubsection{Temperature Programmed Oxidation-TPO and $\mathrm{O}_{2}$ Pulse Chemisorption}

The oxidizing ability of PBCO samples was assessed by $\mathrm{O}_{2}$ temperature programmed oxidation (TPO). The experiments were performed in a Micromeritics Autochem 2910 unit. To measure 
concentration changes, the unit consisted of dual thermal conductivity detectors (TCD) to monitor the change in thermal conductivity between the gas after it flows over the sample and a reference gas stream. The PBCO samples were placed in a $\mathrm{u}$-shaped quartz tube and held in a place by quartz wool. A thermocouple was inserted axially down the tube and was placed in the middle of the sample, or as close as possible depending on the sample size. Before oxidation, the sample underwent a drying treatment step to $200{ }^{\circ} \mathrm{C}$ under an inert gas (Ar) to desorb any surface moisture. Oxidation behavior was examined from room temperature to $700{ }^{\circ} \mathrm{C}$ under a $5 \% \mathrm{O}_{2} / \mathrm{Ar}$ gas mixture by a $5{ }^{\circ} \mathrm{C} / \mathrm{min}$ ramp. The samples were held at $700{ }^{\circ} \mathrm{C}$ for $30 \mathrm{~min}$. Following the isothermal hold, the gas was switched to Ar for 15 minutes to remove any absorbed oxygen. The materials were then cooled to $50{ }^{\circ} \mathrm{C}$ to perform the pulse chemisorption analysis. Once at temperature, Ar was purged over the catalyst, while a $5 \%$ mixture of $\mathrm{O}_{2} / \mathrm{Ar}$ was introduced into a separate line with a loop containing a known volume of $0.5573 \mathrm{ml}$ until the time of injection. Once the pulse was initiated, the loop valve opened and the $\mathrm{O}_{2}$ gas mixture was forced over the sample by the Ar gas. After dosing, the loop valve was closed, and the loop volume was purged with the $5 \% \mathrm{O}_{2} / \mathrm{Ar}$ mixture to prepare for another dose. The dosing process was repeated until the peak areas were equal. In analyzing data, a 1:2 stoichiometric ratio of $\mathrm{O}_{2}$ : metal was used. A calibrated thermal conductivity detector (TCD) is used to determine the number of oxygen molecules taken up by active sites upon injection. Upon saturation no later injections will be chemisorbed. So the number of gas chemisorbed is directly related to the active surface area of PBCO powders.

TPD will be adopted to characterize the oxygen dissociation adsorption mechanism and further be referred to as the oxygen reaction kinetics mechanism on porous PBCO/GDC symmetric cells. To be concrete, it will be used to clarify the oxygen surface exchange process elementary steps. 
Besides, the issue of whether oxygen is adsorbed first on the active site then be dissociated, and reduced into oxygen ions or the three steps are cross-linked with each other can be solved. The key point is that the surface reaction rate on porous PBCO can be experimentally calculated.

\subsubsection{Temperature Programmed Desorption}

Temperature programmed desorption with $\mathrm{O}_{2}$ will be used to identify the multi-site and subsurface, diffusion models. The catalyst sample is exposed to a diluted gas stream containing $\mathrm{N}_{2}$. During the exposure time, the surface of the catalyst is titrated by the reactive gases and becomes saturated with $\mathrm{O}_{2}$ on the active sites. Once the catalyst has been sufficiently saturated the temperature of the sample is elevated by a certain rate to remove the adsorbed $\mathrm{O}_{2}$. The temperature at which the gas desorbs, as well as the size of the peaks, indicates the strength of the interaction and amount of the adsorbed gas.

\subsubsection{Laser Raman}

A Horiba Jobin Yvon LabRam laser Raman confocal microscope was used for the examination of the metal-oxygen bonds in the PBCO materials. All experiments were performed using a 532$\mathrm{nm}$ excitation wavelength which was focused with a 20x magnification objective onto the sample. The laser power exposed to the sample was controlled with a neutral density filter, which was set to $25 \%$. The scattered light reflected from the sample passed through a $400 \mu \mathrm{m}$ confocal hole size after reflecting off a 600-nm grating and was detected by a charge-coupled device (CCD) camera with a spectral resolution of $1 \mathrm{~cm}^{-1}$. Before each experiment, a beam alignment and instrument calibration was performed on a poly-propylene NIST standard, Data was acquired and analyzed using Horiba Labspec 6 software, with an acquisition time of 5s, and a beam accumulation time of $10 \mathrm{~s}$ over a $50-4000 \mathrm{~cm}^{-1}$ spectral range. 


\subsection{Electrical and Electrochemical Characterization}

\subsubsection{Four-point DC Method Conductivity Measurement}

The electrical conductivity was measured using Van der Pauw 4 probe method. After mixing PBCO with 3 wt\% polyvinyl butyral (PVB, Alfa Aesar) uniformly, the mixtures were pressed into pellets (diameter $16 \mathrm{~mm}$ ) and sintered at $1200^{\circ} \mathrm{C}$ for $4 \mathrm{~h}$ in air and nitrogen respectively. PBCO pellets were then polished on both sides using silicon carbide polishing papers and polycrystalline diamond suspension up to $1 \mu \mathrm{m}$ (PACE Technologies, Tucson, Arizona, USA). Two different thicknesses of pellets $(0.5 \mathrm{~mm}$ and $1.5 \mathrm{~mm})$ were obtained for both the assynthesized tetragonal and the as-synthesized orthorhombic PBCO. The porosity of the sintered pellets was measured with Archimedes' principle. First, the weight of dry pellet in the air was measured with analytical balance (A\&D GH-202) and the average value was noted as $\mathrm{M}_{1}$. Second, immersed the pellet in water in a beaker and put the beaker in a vacuum chamber for several minutes to eject air in the pellet. The weight of the saturated pellet was measured again in water and the average weight was noted as $\mathbf{M}_{2}$. The real volume of the pellet can be calculated as $V=\frac{M_{1}-M_{2}}{\rho_{\text {water }}}$. Here, $\rho_{\text {water }}=1 \mathrm{~g} \mathrm{~cm}^{-3}$ is the density of water. Also, the theoretical volume of the pellet can be obtained as $V_{0}=\pi r^{2} h$. Here, $\mathrm{r}$ is the radius and $h$ is the thickness of the pellet. Hence, the porosity ( $\phi$ ) of the pellet can be written as:

$$
\emptyset=\left(1-\frac{V}{V_{0}}\right) \times 100 \%
$$

After sintering, the porosity of all samples was equal to or less than $5 \%$.

Four silver contact points were located at the edge of one side symmetrically. 
The electrical conductivity was measured by the Van de Paul method from room temperature to $700{ }^{\circ} \mathrm{C}$. The sheet resistance can be calculated using $R_{S}=\frac{\pi R}{\ln 2}$, among which $R$ is the resistance. Thus, the conductivity can be expressed as formula (4-8), $\mathrm{L}$ is the thickness of the pellets.

$$
\sigma=\frac{\ln 2}{\pi R L}
$$

\subsubsection{Electrical Conductivity Relaxation (ECR)}

The electrical conductivity relaxation was measured using Van der Pauw 4 probe method on the same pellets described in the conductivity measurement part. The testing was conducted from 500 to $700{ }^{\circ} \mathrm{C}$ across a range of oxygen partial pressure from 0.21 atm to $0.01 \mathrm{~atm}$. For all

conditions, $P_{\mathrm{O}_{2}}$ change follow the rule $\Delta \log P_{\mathrm{O}_{2}} \leq 0.6$. The total flow rate of mixed gas was fixed at $100 \mathrm{sccm}$. The measured surface exchange coefficient $\left(k_{\mathrm{ex}}\right)$ and chemical diffusion coefficient ( $\left.D_{\text {chem }}\right)$ were written as a function of the equilibrium state $P_{\mathrm{O}_{2}}$. The schematic structure of the lab-made ECR instrument set was presented in Figure 4.1. A Keithley 2400 was used to apply constant current and the corresponding voltage was measured by a Keithley $2182 \mathrm{~A}$. The oxygen partial pressure was monitored with an electrochemical oxygen Analyzer (Model 810 Oxygen Analyzer, Illinois Instrument Inc.). Gas flow rate and other operating point measurements were handled by a National Instruments data acquisition controlled with LabVIEW software. The collected ECR curves were fitted by MATLAB with equation ${ }^{171}$ : 

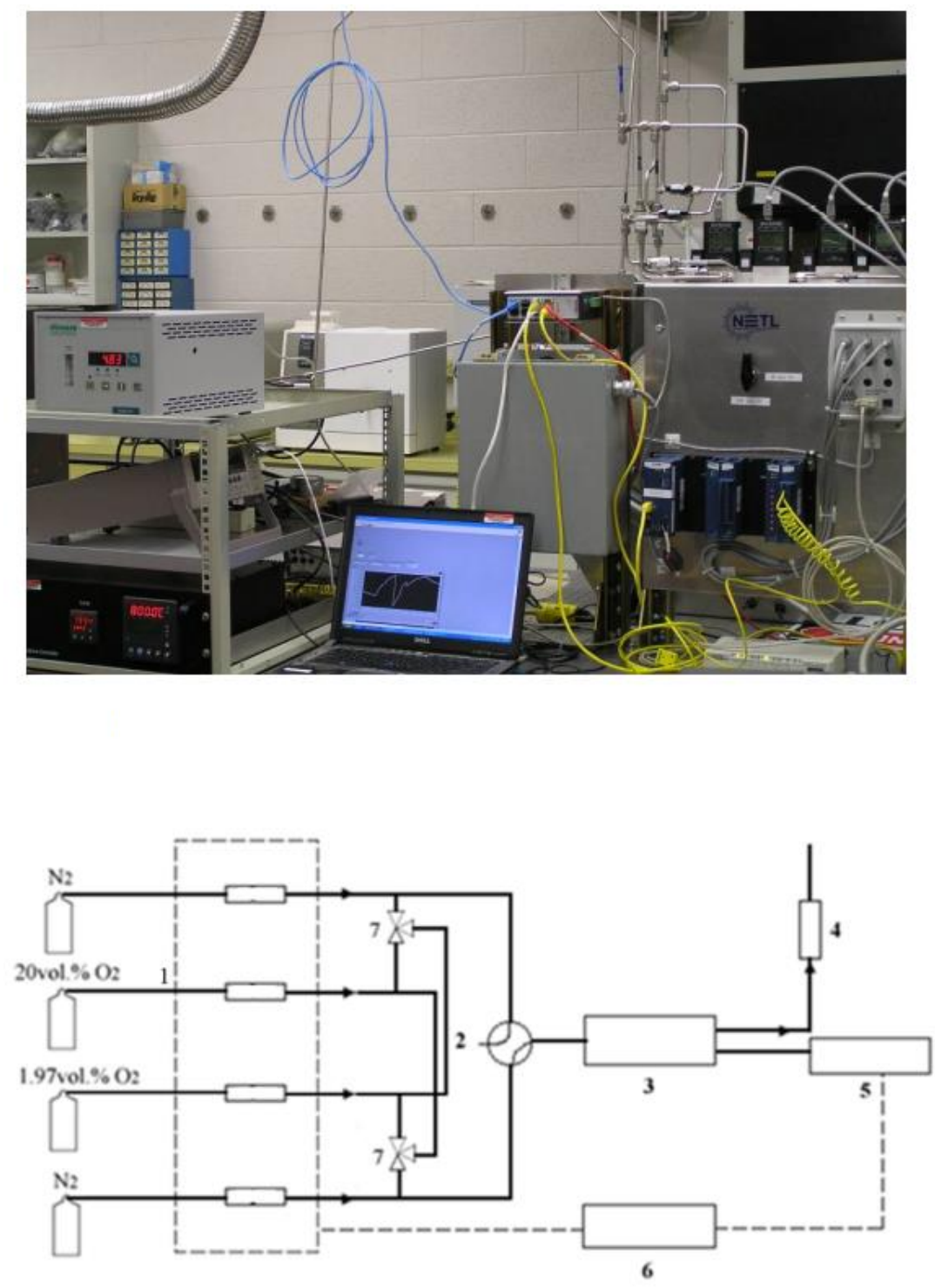

Figure 4.1 Schematic representation of the ECR set-up 1. Mass flow controller, 2. Four-way valve, 3. Furnace, 4. Oxygen analyzer, 5. Nano-voltmeter and current source meter, 6. Computer, 7. Three-way valve.

Before testing on $\mathrm{PBCO}$, calibration of the whole ECR system was made with a $\mathrm{PrBaCo}_{2} \mathrm{O}_{5.68}$ (PBCO) dense pellet sample to ensure the accuracy and reliability of the ECR test. According to den Otter's work ${ }^{172}$, flush time $\left(\tau_{f}\right)$ is the characteristic time needed for new gas to flush the 
reactor chamber, a less flush time is the requirement for more accurate fitting. This parameter can be written as:

$$
\tau_{f}=\frac{V_{r}}{\Phi_{v, t o t}} \times \frac{T_{S T P}}{T_{r}}
$$

In this equation, $\Phi_{v \text {,tot }}$ is the flow rate of gas, $V_{r}$ and $T_{r}$ represent the reactor volume and temperature, respectively, $T_{\mathrm{STP}}$ denotes the room temperature.

\subsubsection{Electrochemical Impedance Spectroscopy (EIS)}

Electrochemical Impedance Spectroscopy (EIS) was carried out on symmetric cells at open circuit conditions over $10^{5}$ to $10^{-2} \mathrm{~Hz}$ frequency range with $10 \mathrm{mV}$ perturbation. EIS was also carried out on three electrodes cells with $0.1 \mathrm{~V}$ cathode polarization over $10^{5}$ to $10^{-2} \mathrm{~Hz}$ frequency. Both the symmetrical cells and the three-electrode cells are testing in air, $0.1 \mathrm{~atm}, 0.04 \mathrm{~atm}$, and $0.01 \mathrm{~atm}$ on both sides of the electrolytes. All electrochemical experiments were performed using an AUTO LAB PGSTAT302N potentiostat/galvanostat (Metrohm, Netherlands) controlled by NOVA 1.11 software. Zview software was used for impedance spectra analysis and fitting. For the symmetric cell, the area-specific resistance (ASR) can be calculated as:

$$
\mathrm{ASR}=\frac{1}{2}\left(R_{\mathrm{p}, \mathrm{m}} \times \mathrm{A}\right)
$$

Where, $R_{\mathrm{p}, \mathrm{m}}$ is the polarization resistance measured from the raw EIS data, $\mathrm{A}$ is the effective region area of one side, the unit of the ASR is $\Omega \mathrm{cm}^{2}$. For the EIS fitting, inductance (L) is involved in the equivalent circuit to mimic a more realistic electrochemical process. This inductance is subtracted in the following data analysis. In the experiments for oxygen reduction reaction on $\mathrm{PBCO}$ cathode investigation, symmetric cells were heated up to $500{ }^{\circ} \mathrm{C}, 550{ }^{\circ} \mathrm{C}, 600$ ${ }^{\circ} \mathrm{C}, 650{ }^{\circ} \mathrm{C}, 700{ }^{\circ} \mathrm{C}$, respectively. Then impedance spectrums were collected every 30 minutes. At 
each target temperature, the cell was soaked for 30 minutes before measurement to ensure a steady status of cells. 


\section{Materials Structure and Performance of LnBCO-Based Cathode}

The crystal structure adopted by $\mathrm{LnBaCo}_{2} \mathrm{O}_{6-\delta}$ has been reported to be either tetragonal $P 4 / \mathrm{mmm}$ $\left(a_{p} \times a_{p} \times 2 a_{p}\right)$, orthorhombic Pmmm $\left(a_{p} \times 2 a_{p} \times 2 a_{p}\right.$ or $\left.a_{p} \times a_{p} \times 2 a_{p}\right)$, or orthorhombic Pmmb $\left(a_{p} \times 2 a_{p} \times 2 a_{p}\right)$. Here $a_{p}$ refers to the basic cubic perovskite cell parameters. The doubling in $\mathrm{c}$ is due to the ordering of $L n$ and $\mathrm{Ba}$ into layers perpendicular to $\mathrm{z}$. The cell doubling in $b$, and the transition from tetragonal to orthorhombic, have been suggested to arise from an ordering of the oxide ion vacancies into channels at a value of $\delta=0.5^{56,173,174}$. There is a close relationship between the crystal structure and the electrochemical performance of the electrodes.

In this chapter, $\mathrm{PrBaCo}_{2} \mathrm{O}_{6-\delta}(\mathrm{PBCO}), \mathrm{NdBaCo}_{2} \mathrm{O}_{6-\delta}(\mathrm{NBCO}), \mathrm{YBaCo}_{2} \mathrm{O}_{6-\delta}(\mathrm{YBCO})$, and $\mathrm{La} 0.5_{5} \mathrm{Ba}_{0.5} \mathrm{CoO}_{3-\delta}$ (LBCO) materials will be discussed. To locate a potential double perovskite cathode, the crystal structure, formation condition, and the structural evolution with temperature and atmosphere are applied to the materials listed. Along with electrochemical performance, the oxygen reduction reaction mechanism will also be investigated.

\subsection{Phase Formation of LnBCO Cathodes}

The calcination condition of LBCO was investigated and the obtained phases were characterized by XRD. Figure 5.1 displayed the XRD patterns of LnBCO calcined in the air. Pure double perovskite structure is obtained for $\mathrm{PBCO}, \mathrm{NBCO}$, and $\mathrm{YBCO}$. According to Rietveld refinement Table 5.1, the as-synthesized $\mathrm{PBCO}, \mathrm{NBCO}$, and $\mathrm{YBCO}$ show a tetragonal structure (P4/mmm space group) with $a \approx a_{p}, b \approx a_{p}, c \approx 2 a_{p}, a_{p}=\AA$, and $\alpha=\beta=\gamma\left(\mathrm{R}_{\mathrm{wp}}=4.67 \%\right.$, $\left.\chi^{2}=1.68 \mathrm{Rp}=4.23 \%\right)$. While the LBCO shows a single perovskite cubic structure $(\mathrm{Pm}-3 \mathrm{~m}$ space group) with $\mathrm{a}=\mathrm{b}=\mathrm{c}=5.489 \AA$. The double perovskite LBCO phase can be obtained by calcination in nitrogen as reported in literature ${ }^{175}$. Figure 5.2 showed the XRD patterns of PBCO, 
NBCO, YBCO, and LBCO calcined in $\mathrm{N}_{2}$. The XRD patterns indicate that the single perovskite remains for $\mathrm{LBCO}, \mathrm{NBCO}$, and $\mathrm{YBCO}$ keeps the double perovskite tetragonal structure. However, the PBCO sintered in nitrogen shows an orthorhombic structure (Pmmm space group) with $a \approx a_{p}, b \approx 2 a_{p}, c \approx 2 a_{p}, a_{p}=3.909 \AA$. The parameters are listed in Table 5.2.

Table 5.1 The refinement structure parameters for PBCO calcined in air.

\begin{tabular}{cccccc}
\hline Atom & $\mathrm{x}$ & $\mathrm{y}$ & $\mathrm{z}$ & Occupancy & Wyck. \\
\hline $\mathrm{Ba}$ & 0.5 & 0.5 & 0 & 1.0 & $1 \mathrm{c}$ \\
$\mathrm{Pr}$ & 0.5 & 0.5 & 0.5 & 1.0 & $1 \mathrm{~d}$ \\
$\mathrm{Co}$ & 0 & 0 & 0.2514 & 1.0 & $2 \mathrm{~g}$ \\
$\mathrm{O} 1$ & 0 & 0 & 0 & 1.0 & $1 \mathrm{a}$ \\
$\mathrm{O} 2$ & 0 & 0 & 0.5 & 0.51 & $1 \mathrm{~b}$ \\
$\mathrm{O} 3$ & 0 & 0.5 & 0.2872 & 1.0 & $4 \mathrm{i}$ \\
\hline
\end{tabular}

Table 5.2 The structure parameters for PBCO calcined in nitrogen.

\begin{tabular}{cccccc}
\hline Atom & $\mathrm{x}$ & $\mathrm{y}$ & $\mathrm{z}$ & Occupancy & Wyck. \\
\hline $\mathrm{O} 1$ & 0 & 0.5 & 0.5 & 0.9 & $1 \mathrm{~g}$ \\
$\mathrm{O} 2$ & 0 & 0 & 0.5 & 0.1 & $1 \mathrm{c}$ \\
$\mathrm{O} 3$ & 0.5 & 0 & 0.306 & 1.0 & $2 \mathrm{~s}$ \\
$\mathrm{O} 4$ & 0.5 & 0.5 & 0.2671 & 1.0 & $2 \mathrm{t}$ \\
$\mathrm{O} 5$ & 0 & 0.2409 & 0.2847 & 1.0 & $4 \mathrm{u}$ \\
$\mathrm{Ba}$ & 0.5 & 0.2469 & 0 & 1.0 & $2 \mathrm{o}$ \\
$\mathrm{Pr}$ & 0.5 & 0.2727 & 0.5 & 1.0 & $2 \mathrm{p}$ \\
$\mathrm{C} 1$ & 0 & 0.5 & 0.25 & 1.0 & $2 \mathrm{r}$ \\
C02 & 0 & 0 & 0.2521 & 1.0 & $2 \mathrm{q}$ \\
O6 & 0 & 0 & 0 & 1.0 & $1 \mathrm{a}$ \\
O7 & 0 & 0.5 & 0 & 1.0 & $1 \mathrm{e}$ \\
\hline
\end{tabular}



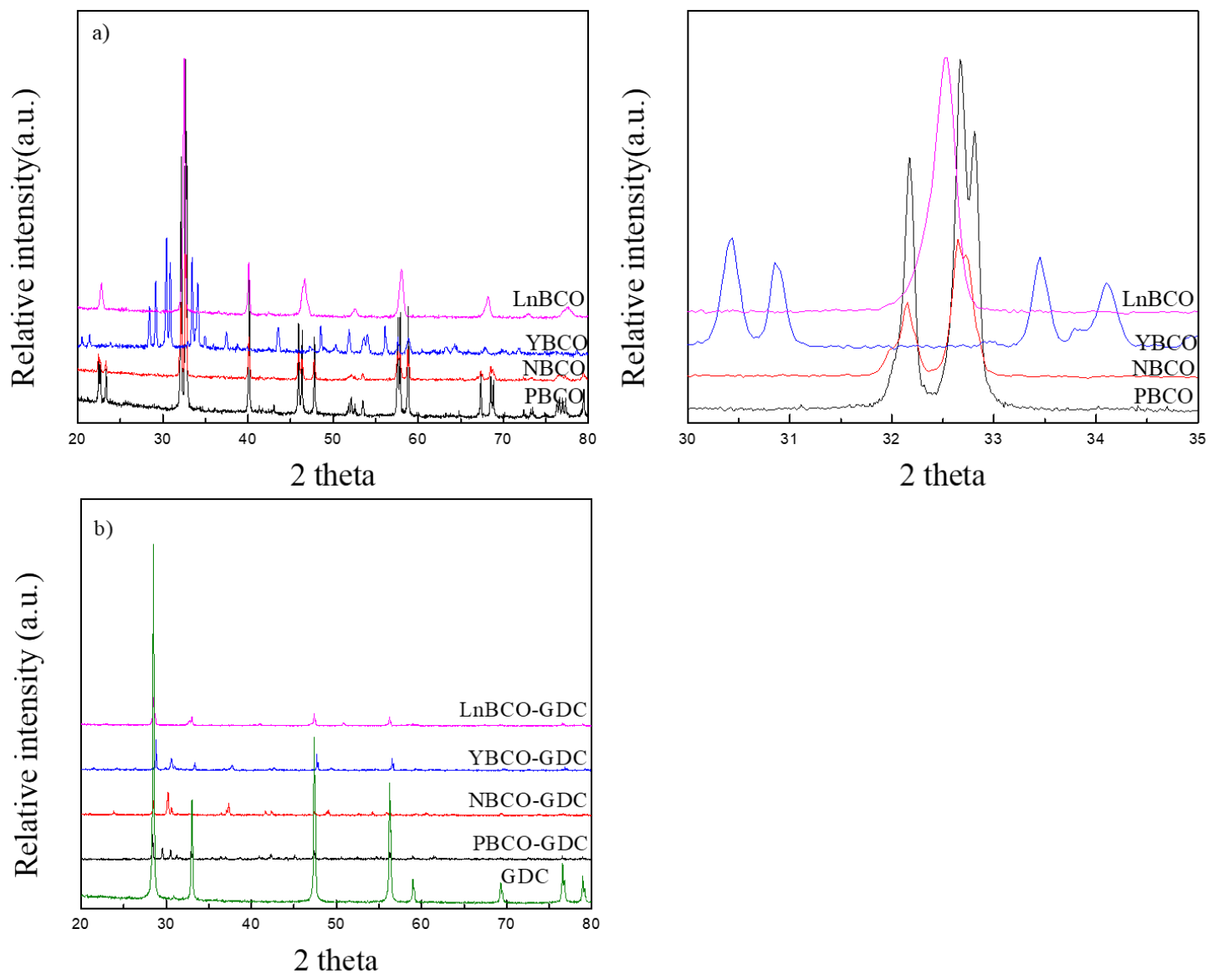

Figure 5.1 XRD patterns a) LnBCO materials calcined in nitrogen, b) compatibility with GDC.
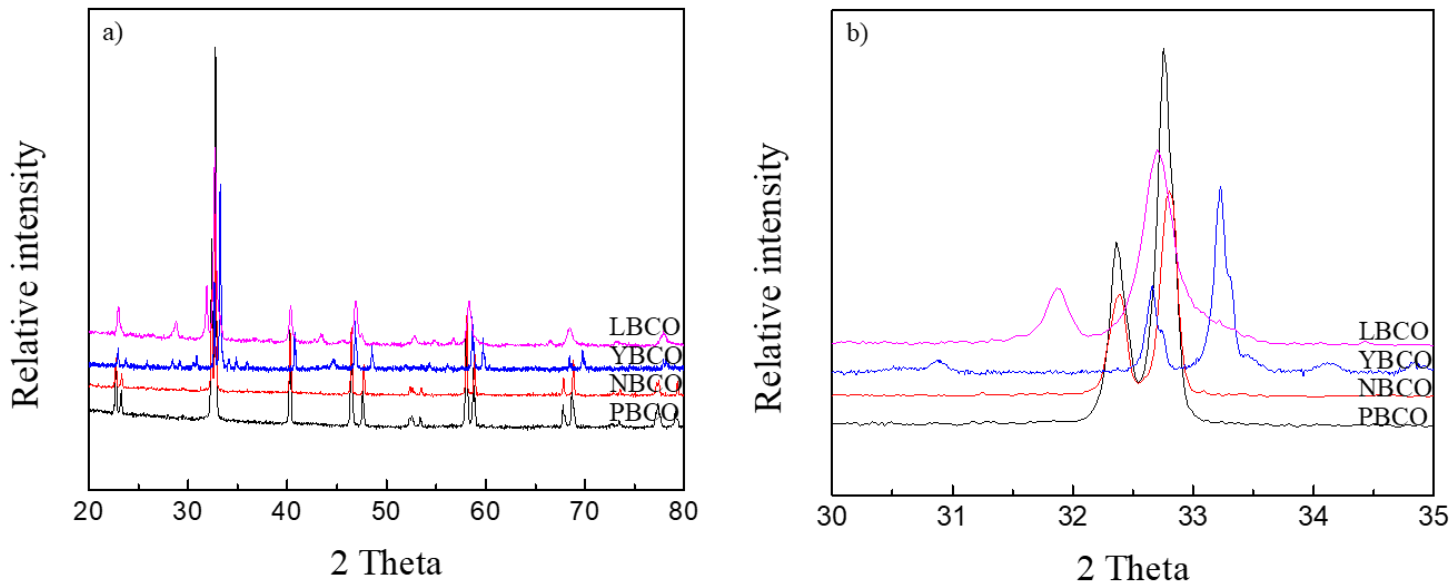

Figure 5.2 XRD patterns of LnBCO calcined in air. 
According to literature ${ }^{13}$, the difference in size and charge between the two A site cations $\left(\operatorname{Ln}^{3+}\right.$ and $\mathrm{Ba}^{2+}$ ) in $\mathrm{LnBCO}$ is the driver for ordering these cations into separate layers. So it is worth explaining that if this driver is insufficient to produce ordering then the materials will simply be A cation disordered. The driving force for ordering the cell into $\mathrm{LnO}_{0.5}$ and $\mathrm{BaO}$ layers is a consequence of $\mathrm{Ba}$ having a significantly larger ionic radius compared to the $\mathrm{Ln}$ cations considered. As the size of $\mathrm{Ln}$ is increased towards La the difference in size compared with the Ba is decreased and the driving force for order decreases. So, the single perovskite $\mathrm{La}_{0.5} \mathrm{Ba}_{0.5} \mathrm{CoO}_{3-\delta}$ is a very stable A cation disordered phase.

To be used as SOFC cathode, the materials must be compatible with the electrolyte. The XRD patterns of the $\mathrm{LnBCO}$, GDC mixtures sintered at $1150{ }^{\circ} \mathrm{C}$ for 12 hours are shown in Figure 5.3. The prepared LnBCO materials proved to be compatible with GDC electrolyte with no additional peaks detected.

\subsection{Electrical Conductivity of LnBCO Cathodes}

The electrical conductivity of LnBCO materials under air and nitrogen was investigated. Figure 5.3 presents the electrical conductivity results of $\mathrm{LnBCO}$ with a temperature increase to $700{ }^{\circ} \mathrm{C}$. This process was taken to investigate the relation of the chemical states on the layered double perovskite surface to their electrical conductivities. The electrical conductivity of PBCO and NBCO decreases with increasing temperature, indicating the nature of metallic characteristics and the values of $\mathrm{PBCO}$ are higher than those of the $\mathrm{NBCO}$. The maximum and minimum conductivities in $\mathrm{PBCO}$ are $1548 \mathrm{~S} / \mathrm{cm}$ at room temperature and $1014 \mathrm{~S} / \mathrm{cm}$ at $700{ }^{\circ} \mathrm{C}$. However, the electrical conductivity values of LBCO initially increase, and then decrease with respect to temperature increase, indicating metal-insulator transition (MIT) behavior. The maximum and 
minimum conductivities in $\mathrm{LBCO}$ are $1095 \mathrm{~S} / \mathrm{cm}$ at $70{ }^{\circ} \mathrm{C}$ and $440 \mathrm{~S} / \mathrm{cm}$ at $700{ }^{\circ} \mathrm{C}$. The maximum electrical conductivity results are caused by the high concentration of electron holes for double perovskite materials. Besides, it is much higher than the ionic conductivity obtained from oxygen vacancies ${ }^{176}$. The concentration of higher valance $\mathrm{Co}^{4+}$ is directly related to the electrical conductivity in the case of the relationship between the surface charge state, investigated by Raman Spectroscopy. The minimum electrical conductivity among the LnBCO materials was $440 \mathrm{~S} / \mathrm{cm}$, which is adequate to be used as a SOFC cathode.
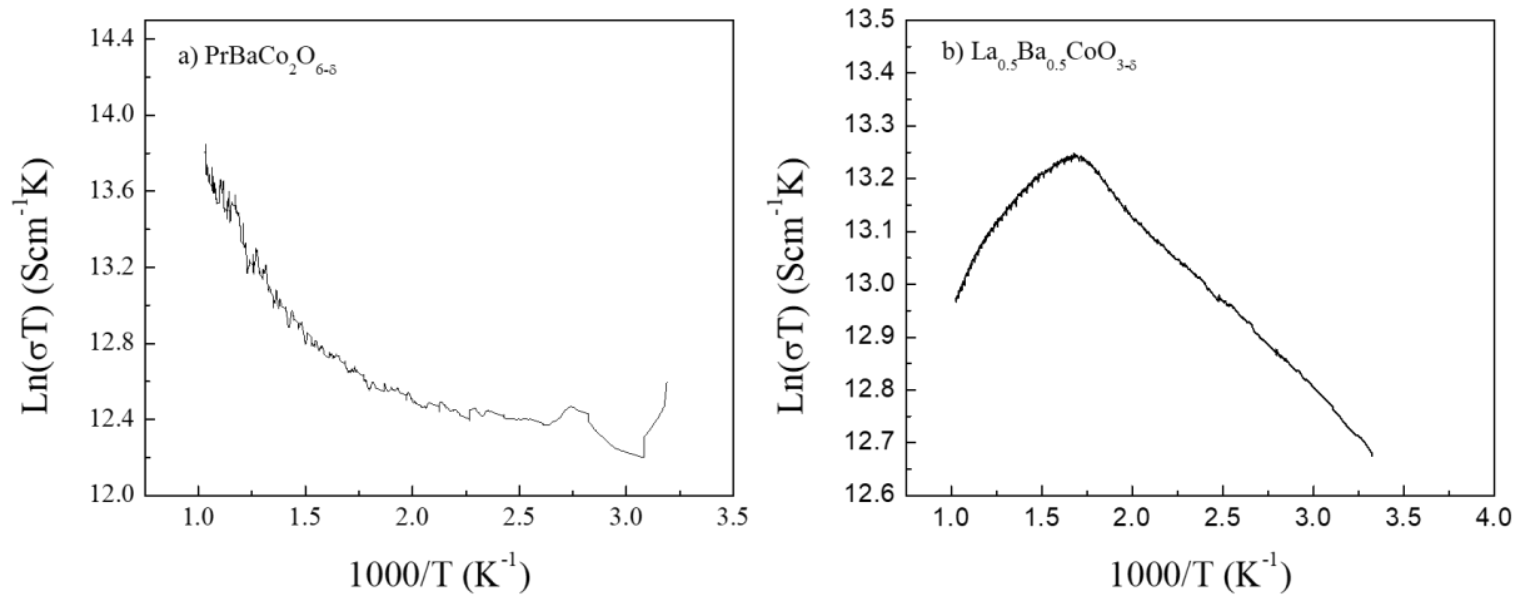

Figure 5.3 Electrical conductivities of LnBCO oxides. a) PBCO, b) LBCO.

There is literature claiming that double perovskite material which belongs to $\mathrm{P} 4 / \mathrm{mmm}$ has little Raman activity, and no specific bands appear under dry conditions ${ }^{177}$. However, the tetragonal PBCO is proved sensitive to Raman shift (see Figure 5.4). The laser Raman can be used as an analytical tool to compliment XRD data. As XRD provides information primarily regarding cation ordering, Raman spectroscopy can detect metal-oxygen bonds and is therefore sensitive to changes in the oxygen sub-lattice. An ideal tetragonal P4/mmm PBCO can have 6 Raman active 
modes, which results from 5 cation-anion vibrations, and 1 oxygen-sublattice only vibration at the highest wavenumber. The vibrational modes for the $P 4 / m m m$ PBCO are represented as ${ }^{178}$ :

$$
\begin{gathered}
\mathrm{Ba}=\mathrm{A}_{2 \mathrm{u}}+\mathrm{E}_{\mathrm{u}} \\
\operatorname{Pr}=\mathrm{A}_{2 \mathrm{u}}+\mathrm{E}_{\mathrm{u}} \\
\mathrm{Co}=\mathrm{A}_{1 \mathrm{~g}}+\mathrm{E}_{\mathrm{g}}+\mathrm{A}_{2 \mathrm{u}}+\mathrm{E}_{\mathrm{u}} \\
\mathrm{O} 1=\mathrm{A}_{2 \mathrm{u}}+\mathrm{E}_{\mathrm{u}} \\
\mathrm{O} 2=\mathrm{A}_{1 \mathrm{~g}}+\mathrm{B}_{1 \mathrm{~g}}+2 \mathrm{E}_{\mathrm{g}}+\mathrm{A}_{2 \mathrm{u}}+\mathrm{B}_{2 \mathrm{u}}+2 \mathrm{E}_{\mathrm{u}} \\
\Gamma_{\text {Raman }}=2 \mathrm{~A}_{1 \mathrm{~g}}+\mathrm{B}_{1 \mathrm{~g}}+3 \mathrm{E}_{\mathrm{g}}
\end{gathered}
$$

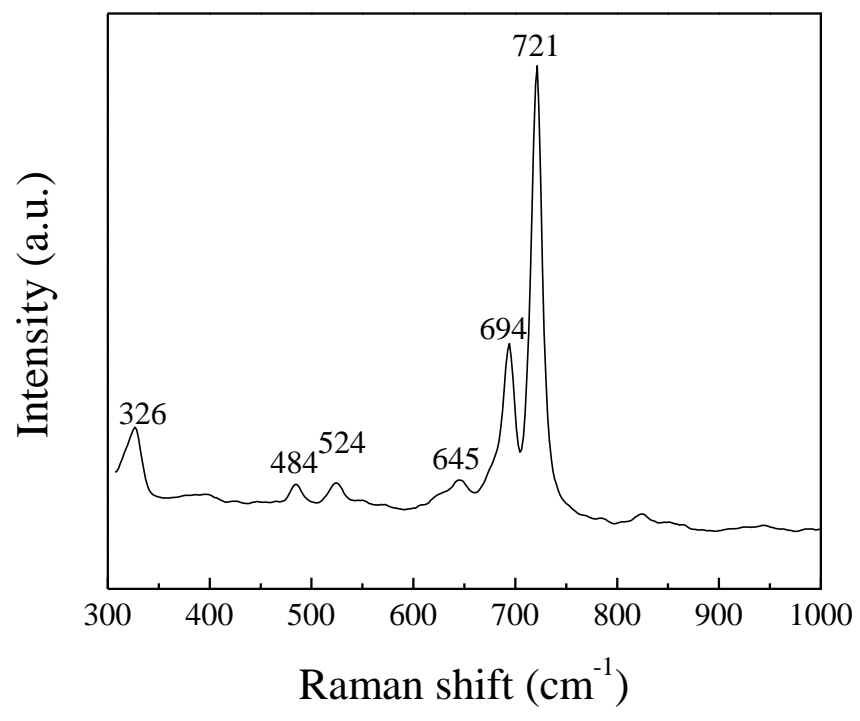

Figure 5.4 Raman Spectra of the PBCO oxide.

The $\mathrm{Pr}, \mathrm{Co}$, and $\mathrm{O} 2$ atoms occupy sites of the same symmetry, whereas the $\mathrm{Ba}$ and $\mathrm{O} 1$ vibrations are Raman forbidden telling from the $\Gamma_{\text {Raman. }}$ The broad spectrum with a slight peak around 645 
$\mathrm{cm}^{-1}$ is believed to correspond to the Raman active mode of the anion disordered $P 4 / \mathrm{mmm}$ phase, with the broad profile indicating the anions are randomly ordered. The strongest and most characteristic peak at $\sim 721 \mathrm{~cm}^{-1}$ is associated with the $\mathrm{E}_{\mathrm{g}}$ mode designating the $\mathrm{O}(2)-\mathrm{Co}-\mathrm{O}(2)$ bending in the BO6 octahedra. Three other distinguishing bands also become noticeable at 326 $\mathrm{cm}^{-1}, 484 \mathrm{~cm}^{-1}$, and $524 \mathrm{~cm}^{-1}$. These are defined as the respective cation-anion bonds corresponding to the $3 \mathrm{E}_{\mathrm{g}}$ stretching bands, $\mathrm{Pr}-\mathrm{O}(1), \mathrm{Pr}-\mathrm{O}(2), \mathrm{Co}-\mathrm{O}(2)$ that have the different cation-anion bonds which increase in bond strength.

\subsection{Oxygen Reduction Reaction Performance of $\mathrm{LnBCO}$ cathodes}

\subsubsection{Symmetrical Cell Performance of LBCO}

One layer screen printing corresponds to around $10 \mu \mathrm{m}$ after sintering at $1000{ }^{\circ} \mathrm{C}$ for 2 hours with the cathode slurry prepared. The thickness of the porous electrode is about $30 \mu \mathrm{m}$, as illustrated in the represented PBCO cell morphology (Figure 5.5). As clarified in the cell fabricate the part, silver paste and wire instead of platinum paste and wire were used as the current collector to avoid the electrocatalytic promotion from the platinum deposited at the electrode/electrolyte interface.
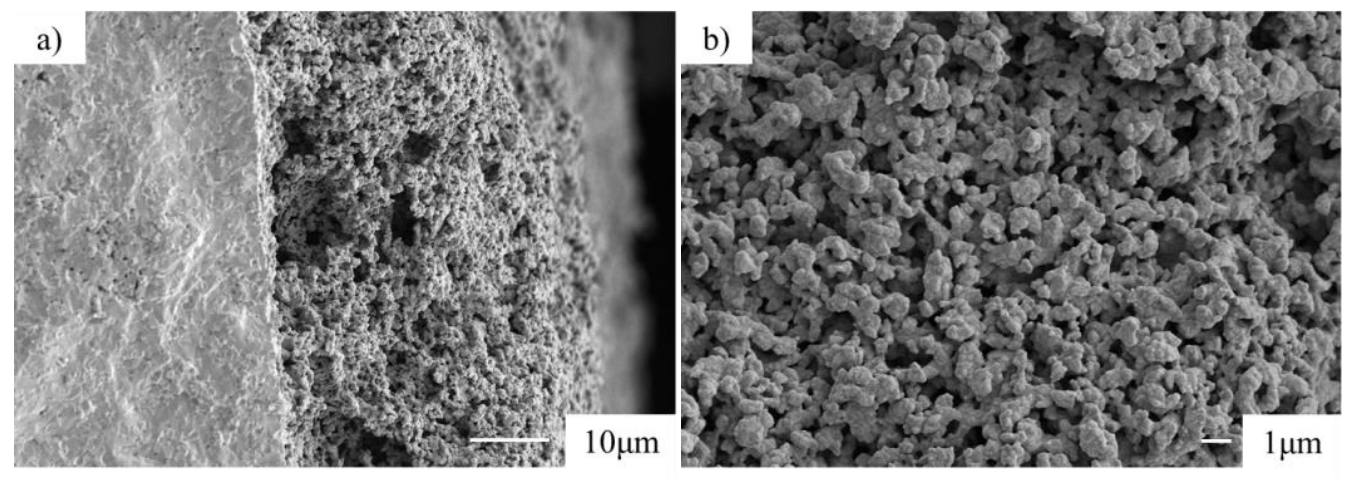

Figure 5.5 SEM morphology of a) the cross-section, b) the porous electrode of the PBCO/GDC/PBCO symmetrical cell. 
The open circuit voltage (OCV) polarization resistance $\left(R_{\mathrm{p}}\right)$ of cathode material in SOFC is an effective parameter to assess the catalytic activity on the oxygen reduction reaction (ORR). Figure 5.6 shows the Nyquist plots of $\mathrm{LnBCO} / \mathrm{GDC} / \mathrm{LnBCO}$ symmetrical cells from $500{ }^{\circ} \mathrm{C}$ to $700{ }^{\circ} \mathrm{C}$ in the air. An attractive polarization resistance is already obtained at $600{ }^{\circ} \mathrm{C}$ for PBCO as $0.165 \Omega \mathrm{cm}^{2}$. An excellent performance was also observed for the YBCO cathode with polarization resistance $0.16 \Omega \mathrm{cm}^{2}$ at $650^{\circ} \mathrm{C}$. These performances are prominent compared with other double perovskite materials like $\mathrm{EuBaCo}_{2} \mathrm{O}_{6-\delta}$ and $\mathrm{SmBaCo}_{2} \mathrm{O}_{6-\delta}$. The performance can also compete with other thin film and composite cathode like LSC, LSCF, and BSCF listed in Table 5.3 .

Table 5.3 Comparison of polarization resistance of ceramic oxide cathodes in air.

\begin{tabular}{llll}
\hline Material & $R \mathrm{p}\left(\Omega \mathrm{cm}^{2}\right)$ & Temperature $\left({ }^{\circ} \mathrm{C}\right)$ & Ref. \\
\hline $\mathrm{La}_{0.6} \mathrm{Sr}_{0.4} \mathrm{CoO}_{3} / \mathrm{YSZ}$ & 0.1 & 600 & 20 \\
$\left(\mathrm{Ba}_{0.5} \mathrm{Sr}_{0.5}\right)_{0.99} \mathrm{Co}_{0.8} \mathrm{Fe}_{0.2} \mathrm{O}_{-\delta}$ & 0.16 & 600 & 21 \\
$\mathrm{LSC}$ thin film/GDC & 0.13 & 600 & 22 \\
$\left(\mathrm{La}_{0.6} \mathrm{Sr}_{0.4}\right)_{1-\mathrm{x}} \mathrm{Co}_{0.2} \mathrm{Fe}_{0.8} \mathrm{O}_{3+} \mathrm{Ce}_{0.9} \mathrm{Gd}_{0.1} \mathrm{O}_{3}$ & 0.19 & 600 & 23 \\
$\mathrm{LSM}+\mathrm{YSZ}$ & 0.14 & 800 & 24 \\
$\mathrm{PrBa}_{0.5} \mathrm{Sr}_{0.5} \mathrm{Co}_{2-\mathrm{x}} \mathrm{Fe}_{\mathrm{x}} \mathrm{O}_{6-\delta}$ & 0.07 & 600 & 25 \\
$\mathrm{EuBaCo}_{2} \mathrm{O}_{6-\delta}$ & 0.5 & 600 & 26 \\
$\mathrm{LSCF} / \mathrm{YSZ}$ & 0.33 & 600 & 27 \\
$\mathrm{SmBaCo}_{2} \mathrm{O}_{6-\delta}$ & 0.48 & 700 & 28 \\
\hline
\end{tabular}

As shown in Figure 5.6a and 5.6d, the impedance spectroscopy of PBCO, NBCO, and LBCO symmetrical cells are a half-tear-drop-shaped impedance which is composed of a diagonal of about $45^{\circ}$ at the high-frequency region followed by a semi-circle at the low-frequency region. The half-tear-drop-shaped impedance appeared is characteristic of a co-limitation of surface oxygen exchange and oxygen bulk diffusion in the oxygen reduction reaction ${ }^{40}$. When the 
electrode presents a chemical solid-state diffusion and the electrochemical process is only limited by the oxygen exchange at the surface, the impedance is composed of two semicircle-like shapes. However, if solid-state diffusion is slowed down or surface oxygen exchange rate is promoted, at some point the electrochemical process will be co-limited by the surface reaction and the bulk diffusion steps. In that case, the impedance will transform from a semicircle or semicircle-like shape to a half-tear-drop shape. This co-limitation process can be described as:

$$
\begin{gathered}
Z_{\text {chem }}=R_{\text {chem }} \sqrt{\frac{1}{1+j w t_{\text {chem }}^{\alpha}}} \\
C_{\text {chem }}=\frac{t_{\text {chem }}}{R_{\text {chem }}} \\
f=\frac{\left(-\partial \mu_{O_{2}} / \partial \delta\right)}{R T} \\
C_{\text {chem }}=\frac{4 F^{2}(1-\varepsilon) l_{\delta}}{R T f V_{m}}=\frac{4 F^{2}(1-\varepsilon) l_{\delta}}{f V_{m}}\left(-\frac{\partial \mu_{O_{2}}}{\partial \delta}\right)^{-1}
\end{gathered}
$$

Where $Z_{\text {chem }}$ is the chemical impedance co-limited by surface oxygen exchange and solid-state diffusion. $R_{\text {chem }}$ is the characteristic resistance from the chemical reaction. $t_{\text {chem }}$ is the characteristic time. $f$ is a thermodynamic factor reflecting the ease with which to change the stoichiometry $(\delta)$ in a given change of $P_{\mathrm{O}_{2}} \cdot \varepsilon$ is the porosity of the electrode. $l_{\delta}$ is the characteristic "utilization" length which indicates the thickness of the active region in a porous electrode, normally at a range of several micrometers. $V_{\mathrm{m}}$ is the molar volume of the electrode. $C_{\text {chem }}$ is the chemical capacitance related to the bulk oxidation/reaction of the material and usually very large. For a very thin $(1.5 \mu \mathrm{m}) \mathrm{La}_{0.6} \mathrm{Sr}_{0.4} \mathrm{CoO}_{3-\delta}$ mixed-conducting film the chemical capacitance is about $0.1-1 \mathrm{~F} \mathrm{~cm}^{-2}{ }^{179}$. This value is about three orders larger than the oxygen 
adsorption and transport dominated pseudocapacitance of $\mathrm{Pt}\left(\sim 10^{-3} \mathrm{Fcm}^{-2}\right)$ and five orders larger than the interfacial polarization capacitance of the Pt/YSZ interface $\left(10^{-6}-10^{-5} \mathrm{Fcm}^{-2}\right)$.

The co-limited theory was applied to the double perovskite LnBCO materials. Impedances of YBCO symmetrical cells are fitted well (Figure 5.6c) with R1(R2CPE1) and R1(R2CPE1) (R3CPE2) equivalent circuits. R1 represents ohmic resistance from electrolyte and electrode ${ }^{180}$. The semicircle-like impedance in the high-frequency region corresponds to the oxygen ion transfer process at the electrode/electrolyte interface (R2CPE1) ${ }^{181}$. The semicircle-like impedance (R3CPE2) in the low-frequency region presents the oxygen bulk diffusion and surface chemical exchange ${ }^{182}$. Impedances of PBCO NBCO and LBCO symmetrical cells are fitted well with the Gerischer element whose impedance is defined by Equ. 5-1 ${ }^{183}$. The half-teardrop curve (Figure 5.6a, 5.6b, and Figure 5.6d) in the low-frequency region presents the oxygen bulk diffusion and surface chemical exchange (gas adsorption, dissociation, and oxygen exchange) co-limited process ${ }^{184}$. As expected, $R_{\text {chem }}$ decreases as the temperature increases, which implies faster surface kinetics at a higher temperature. Chemical capacitance $C_{\text {chem }}$ calculated from the fitting result is very large $\left(1-1.3 \mathrm{~F} \mathrm{~cm}^{-2}\right)$ which accords with Kawada's result 179. From the proportional relationship between $C_{\text {chem }}$ and $l_{\delta}\left(C_{\text {chem }} \propto l_{\delta}\right)$ from Eq. (5-2), the increase of $C_{\text {chem }}$ with temperature illustrates an expansion of the active region in the porous electrode. Furthermore, the size of the active region is estimated. Calculated from Rietveld refinement, the molar volume $V_{\mathrm{m}}$ of this material is $70.28 \mathrm{~cm}^{3} \mathrm{~mol}^{-1}$. The porosity of the electrode is 0.25 . The accurate active length will be calculated after the thermodynamic factor is determined in Chapter 6. Here, the length is estimated with the thermodynamic factor for the layered materials reported in literature ${ }^{185-187}$, the thermodynamic factor $\mathrm{f}$ is estimated as 10 . From Eq. (5-4) the size of the active region $l_{\delta}$ is about $15 \mu \mathrm{m}$ at $700{ }^{\circ} \mathrm{C}$ and 3 to 5 times the 
typical values $(3-5 \mu \mathrm{m}){ }^{10}$. This value demonstrates that a large portion of the electrode surface is active for ORR, which indicates excellent ionic conductivity, good catalytic activity, and a sufficient electrical conductivity of PBCO.
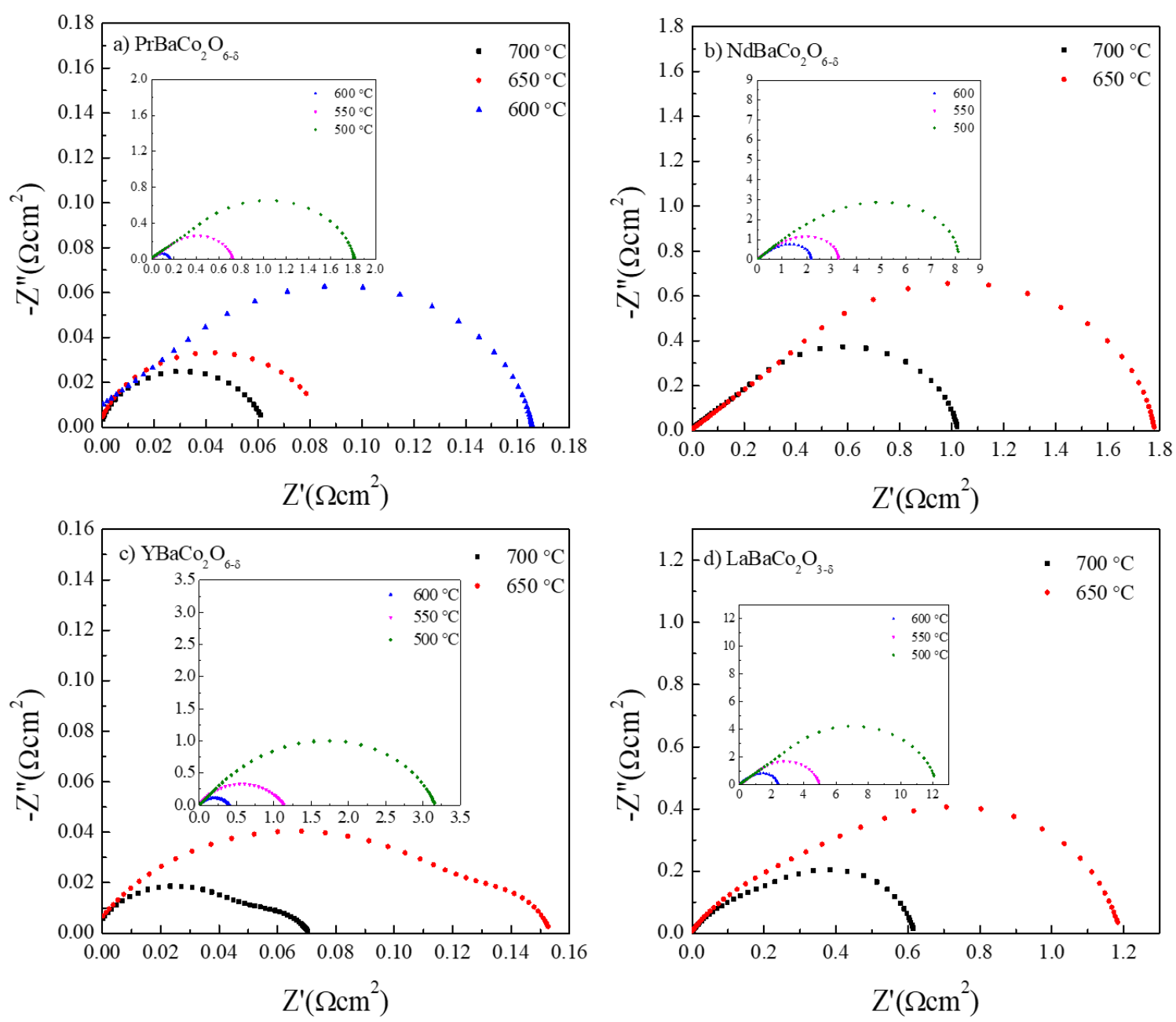

Figure 5.6 Nyquist plot of $\mathrm{LnBCO}$ symmetrical cells from $700{ }^{\circ} \mathrm{C}$ to $500{ }^{\circ} \mathrm{C}$. a) $\mathrm{PBCO}$, b) $\mathrm{NBCO}$, c) YBCO, d) LBCO.

The Nyquist plots of $\mathrm{YBCO} / \mathrm{GDC} / \mathrm{YBCO}$ symmetrical cell at $500{ }^{\circ} \mathrm{C}$ and $700{ }^{\circ} \mathrm{C}$ in oxygen partial pressures $0.21 \mathrm{~atm}, 0.1 \mathrm{~atm}, 0.04 \mathrm{~atm}$, and $0.01 \mathrm{~atm}$ are shown in Figure 5.7a. The activation energy $\left(E_{\mathrm{a}}\right)$ in the air is $1.23 \mathrm{eV}$, in $0.01 \mathrm{~atm}$ is $0.82 \mathrm{eV}$. The capacitance calculated is 
in $\mathrm{mF} \mathrm{cm}{ }^{-2}$ degree. The reaction order is between 0.14 and 0.41 . Different reaction models have been developed in literature and may lead to reaction order in specific scenarios. However, in general, the processes dependent on $P_{\mathrm{O}_{2}}$ less than $1 / 4$ are most likely attributed to the charge transfer process and those displaying more than $1 / 2$ are often attributed to processes involving diatomic oxygen species ${ }^{188-190}$.
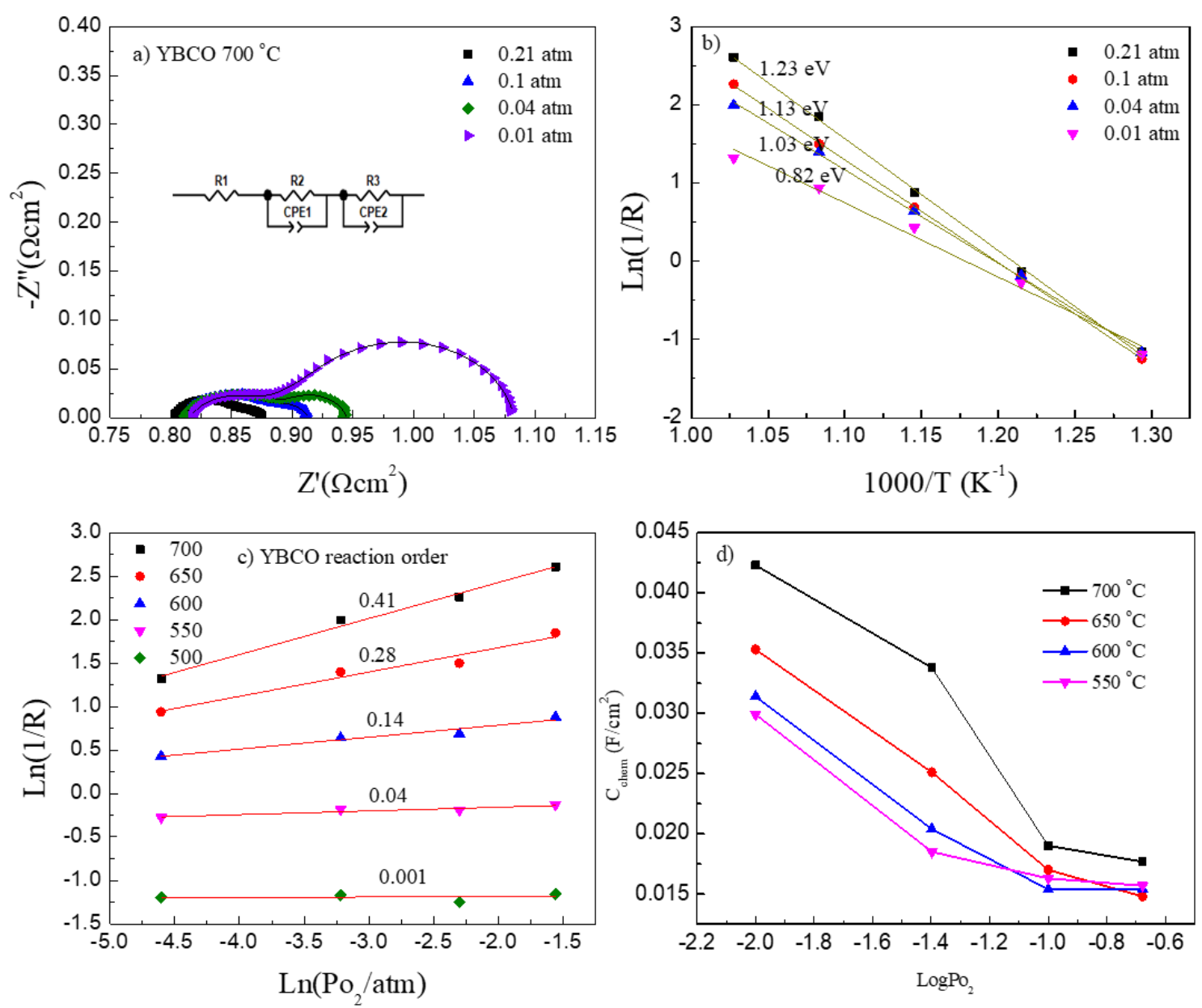

Figure 5.7 Symmetrical cell of YBCO tested in different oxygen partial pressure: a) Nyquist plots of impedance at $700{ }^{\circ} \mathrm{C}$ and fitting line. b) Arrhenius plot and activation energy of the total cathode polarization resistance. c) Polarization resistance as a function of partial pressure and reaction order. d) $C_{\text {chem }}$ as a function of partial pressure. 
Judging from the reaction order, the process associated with the low-frequency arc at $700{ }^{\circ} \mathrm{C}$ is attributed to the oxygen dissociative adsorption or surface diffusion of atomic oxygen, but not a charge transfer process, Since the capacitance of the electrode, is in the order of $\mathrm{mF}$ which is larger than those associated with the charge transfer process, typically $10^{-3} \sim 10^{-1} \mathrm{mF} \mathrm{cm}^{-2}$. The surface diffusion process could possibly arise considering the $1.23 \mathrm{eV}$ activation energy similar to that reported in the literature ${ }^{135,191}$. In the literature, the medium frequency arc with Ea of 1.3 $\mathrm{eV}$ and $2 \mathrm{eV}$ activation energy was related to the surface diffusion of $\mathrm{O}^{-}$.

Cathodic overpotential is an important component of electrode performance. To further investigate the ORR mechanism in the cell operation condition. Three-electrode cells were constructed as stated in the cell fabrication method in chapter 4. The three-electrode configuration allowed direct measurement of the half-cell voltage with and without an applied direct overpotential ${ }^{192-195}$. The cells were tested both under $0.1 \mathrm{~V}$ cathode polarization and the OCV conditions in the air using the EIS technique. Figure 5.8a shows the Nyquist plots of PBCO three-electrode cell with $0.1 \mathrm{~V}$ over-potential. Figure $5.8 \mathrm{~b}$ and $5.8 \mathrm{c}$ show the Nyquist plots of the three-electrode tested in cathodic overpotential and the OCV conditions. The measured value dropped from $0.4 \Omega \mathrm{cm}^{2}$ to $0.13 \Omega \mathrm{cm}^{2}$ at $700{ }^{\circ} \mathrm{C}$ under $0.1 \mathrm{~V}$ cathode polarization.

The derived activation energy of the cell tested under OCV is $1.25 \mathrm{eV}$ which is in the range of the oxygen surface exchange determining step. Specifically, it is known that oxygen adsorption and desorption rates can differ significantly, with desorption occurring much more slowly than adsorption ${ }^{196}$. In the absence of an applied voltage, there is no net ion flux, and thus the adsorption and desorption processes contribute equally to the measured resistance. Under a cathodic over-potential, however, net adsorption occurs at the electrode functioning as the cathode, and the measured rate is dominated by this faster process, resulting in the observed 
reduction in ASR. Such an effect could not occur for an overall process limited by bulk diffusion. The increase of the apparent activation energy when applied overpotential can be convoluted to the enlarging activated electrocatalytic region of the electrode. The applied overpotential can affect the local chemical composition of an MIEC, therefore the accompanying surface coverage. It can also change the surface diffusion, therefore the available site to 3PB.
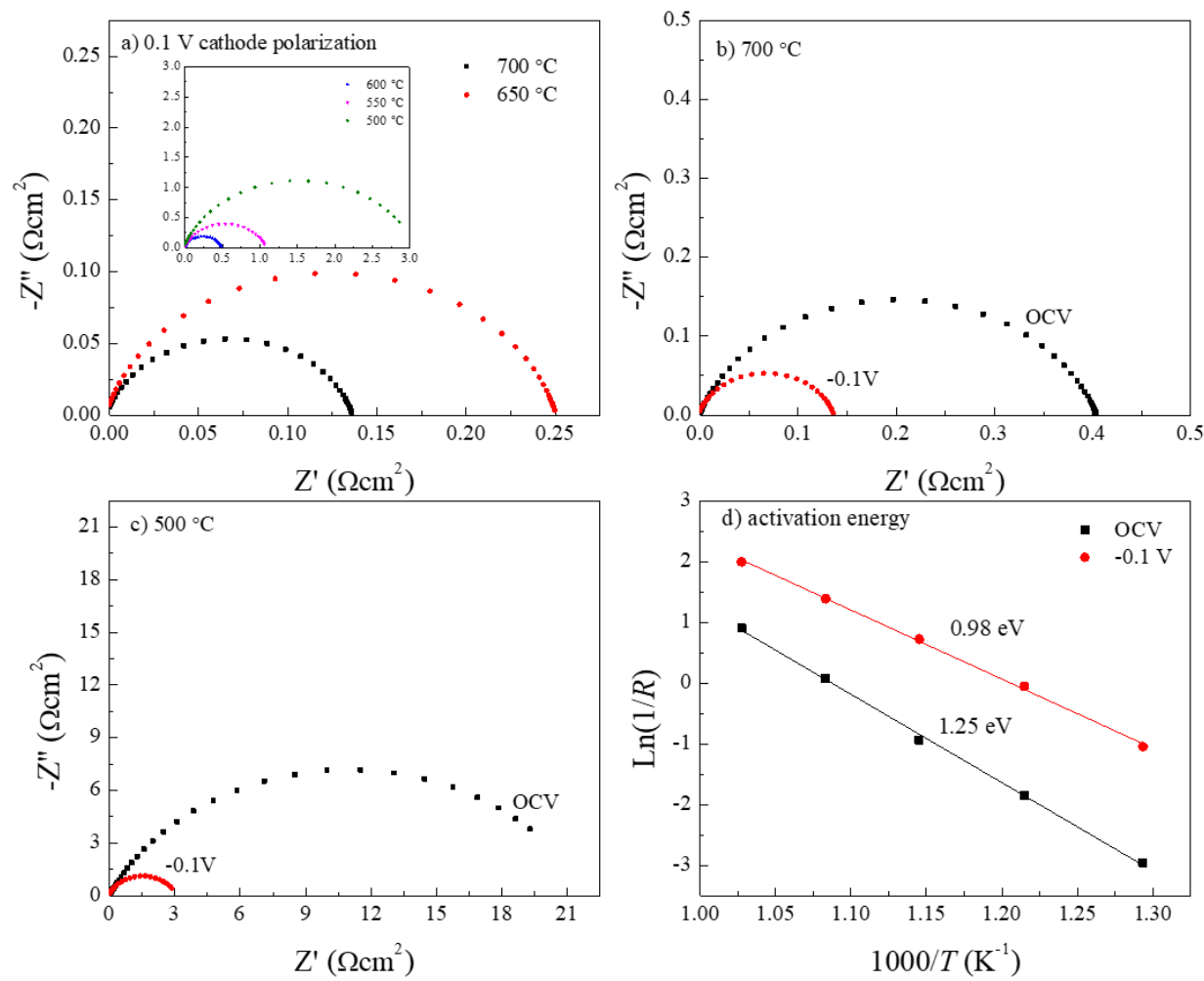

Figure 5.8 Nyquist plots of PBCO three-electrode cells. a) tested in $0.1 \mathrm{~V}$ cathodic overpotential. b) and c) comparison between $0.1 \mathrm{~V}$ cathodic polarization and OCV conditions. d) derived activation energy.

\subsection{Summary}

In this chapter, $\mathrm{PrBaCo}_{2} \mathrm{O}_{6-\delta}(\mathrm{PBCO}), \mathrm{NdBaCo}_{2} \mathrm{O}_{6-\delta}(\mathrm{NBCO}), \mathrm{YBaCo}_{2} \mathrm{O}_{6-\delta}$ (YBCO), and $\mathrm{La}_{0.5} \mathrm{Ba}_{0.5} \mathrm{CoO}_{3-\delta}(\mathrm{LBCO})$ materials are synthesized and investigated to develop a potential solid oxide fuel cell cathode with good electrochemical performance. Phase stability in air and 
nitrogen are studied. Except for LBCO, the other three materials exhibit stable double perovskite with all tetragonal structures in the air. PBCO material prepared in nitrogen presents a double perovskite with an orthorhombic structure. The XRD lattice parameters of the materials synthesized proved that the tetragonal and orthorhombic $\mathrm{LnBCO}$ except for $\mathrm{La}_{0.5} \mathrm{Ba}_{0.5} \mathrm{CoO}_{3-\delta}$ are layered materials.

The electrical conductivity test proves that the PBCO material has a metallic characteristic with the electrical conductivity decreases with temperature increase. The LnBCO material shows a metal-insulator transition behavior. The minimum electrical conductivity of both the PBCO and LBCO materials can meet the SOFC cathode requirement. The maximum electrical conductivity is connected with the higher concentration of electron holes and the layered perovskites structures.

The half-tear-drop-shaped impedance spectra of PBCO and semicircle-like shaped impedance spectra of YBCO symmetrical cells herald PBCO and YBCO are good MIEC cathode which possesses both excellent oxygen surface exchange and bulk diffusion performance. The good match of these two reactions expanded the reaction active zone from electrolyte/electrode $2 \mathrm{~PB}$ to the gas/electrode surface area. This is also supported by the chemical capacitance $\left(1 \mathrm{~F} / \mathrm{cm}^{2}\right.$ to 10 $\left.\mathrm{F} / \mathrm{cm}^{2}\right)$ which is thousand times that of double-layer capacitance $\left(\sim 1 \mathrm{mF} / \mathrm{cm}^{2}\right)$. The bulk diffusion and surface exchange process at gas/electrode $2 \mathrm{~PB}$ was assumed to co-limit the ORR on the PBCO cathode. The oxygen dissociative adsorption and surface diffusion of $\mathrm{O}^{-}$are the ratedetermining step of ORR on the YBCO cathode. 


\section{The structure Evolution of $\mathrm{PrBaCo}_{2} \mathrm{O}_{6-\delta}$ and the Influence on the Thermodynamic Property}

Oxygen transport plays a critical role in SOFC. High oxygen ion conductivity materials are necessary to facilitate oxygen diffusion and promote electro-catalytic performance, especially for SOFC. Double perovskite materials with substantial catalytic activity have been studied a lot, including transport mechanisms and better energy conversion efficiency. $\mathrm{PrBaCo}_{2} \mathrm{O}_{6-\delta}$ cation ordered perovskite is outstanding among LnBCO materials for its excellent oxygen electrocatalytic performance. Both tetragonal and orthorhombic $\mathrm{PrBaCo}_{2} \mathrm{O}_{6-\delta}$ consist of cation ordered sub-lattice. However, the performance of orthorhombic PBCO, which has a close relationship to tetragonal PBCO has not been studied yet. It is already certified that PBCO will undergo a phase transition from orthorhombic to tetragonal. Thus, it is necessary to compare oxygen nonstoichiometry values and the oxygen vacancy concentration, relate them to PBCO structure evolution, and compare the thermodynamic property of the as-synthesized tetragonal and orthorhombic PBCO to further facilitate the oxygen transport and ORR mechanism study.

\subsection{The Lattice Structure of PBCO and Refinement}

Fig. 6.1a and $1 \mathrm{~b}$ show the XRD of PBCO powders sintered in nitrogen and air respectively. The room temperature diffraction peaks can be indexed to the orthorhombic Pmmm space group and tetragonal $\mathrm{P} 4 / \mathrm{mmm}$ space group for the two kinds of powders. Both PBCO diffraction patterns have split peaks at principle planes, which means they belong to double perovskite $\mathrm{PBCO}{ }^{55}$. For tetragonal $\mathrm{PBCO} a \approx b, c \approx 2 a$.Orthorhombic PBCO have the additional peaks (l $\left.\begin{array}{lll}0 & 1 & 0\end{array}\right),\left(\begin{array}{lll}0 & 1 & 2\end{array}\right)$,

(l0 2 0), (l $\left.\begin{array}{lll}1 & 2 & 0\end{array}\right)\left(\begin{array}{lll}0 & 2 & 2\end{array}\right)$ and (ll 22 2) compared to tetragonal PBCO. This lead to the expansion of lattice along the $\mathrm{b}$ axis and the much more ordered oxygen transport in the ab plane compared to 
the tetragonal phase. The peaks shift left entirely with temperature increase, indicate lattice expansion for both samples. This is caused by the reduction of cobalt valence ${ }^{197}$, which increases oxygen vacancy concentration. A phase transition from orthorhombic to tetragonal occurred at $550{ }^{\circ} \mathrm{C}$ and keeps with the temperature above $550{ }^{\circ} \mathrm{C}$.
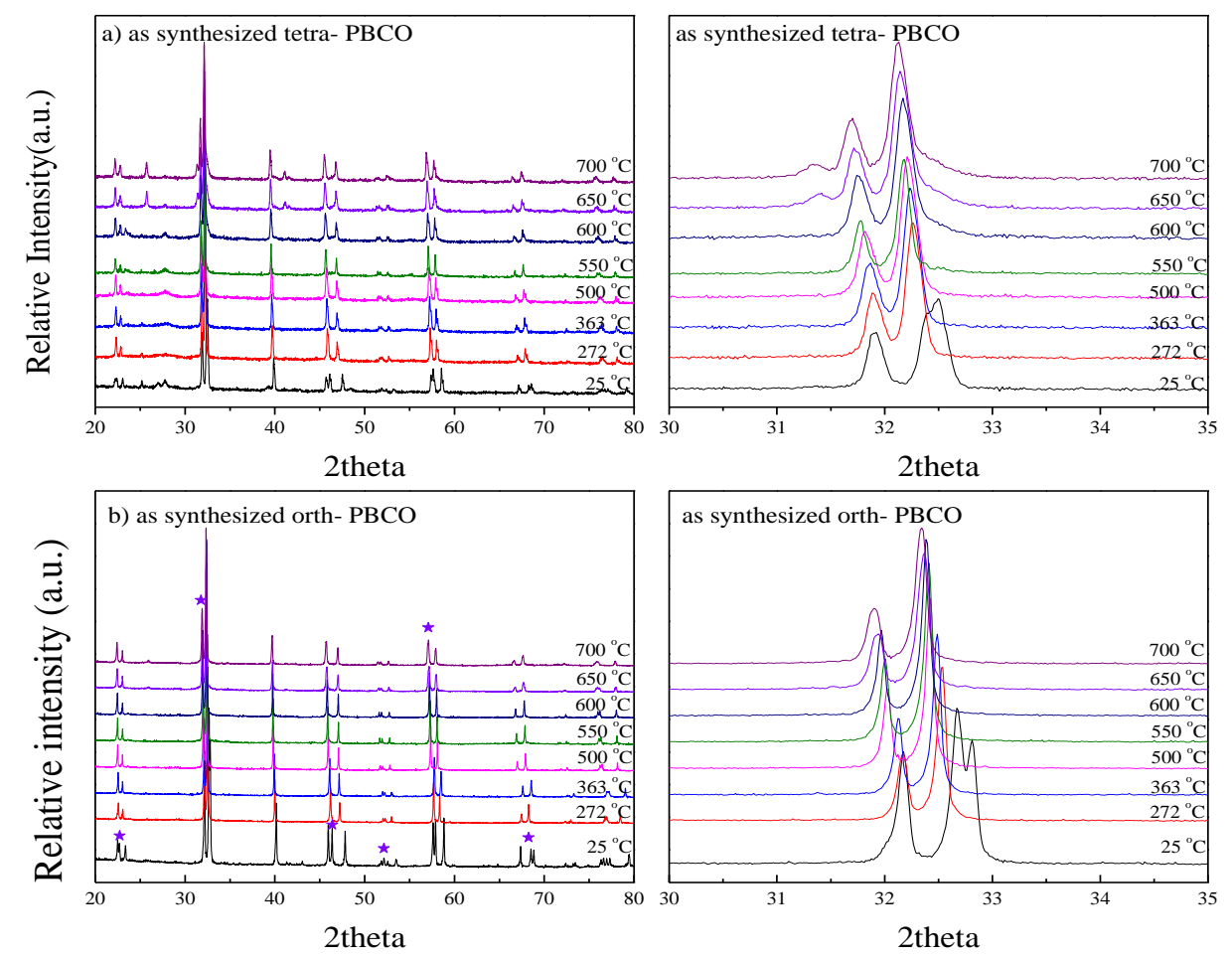

Figure 6.1 X-ray diffraction patterns of PBCO: (a) pristine tetragonal, (b) pristine orthorhombic.

The lattice parameters of the two kinds of as-synthesized PBCO in the temperature range from $500{ }^{\circ} \mathrm{C}$ to $700{ }^{\circ} \mathrm{C}$ are listed in Table 6.1. There is a narrow difference in lattice parameters for these two different original PBCO structures, with $a_{\text {tetra }}<a_{\text {orth }}, c_{\text {tetra }}>c_{\text {orth }}$ when above $550^{\circ} \mathrm{C}$. It is supposed that oxygen transport through pristine tetragonal PBCO will be faster than that in as-synthesized orthorhombic PBCO. This is caused by the faster oxygen diffusion along the ab plane than the $\mathrm{c}$ axis ${ }^{187}$. 
Table 6.1 Lattice parameters evolution of pristine tetragonal and orthorhombic PBCO.

\begin{tabular}{llllll}
\hline Temperature $/{ }^{\circ} \mathrm{C}$ & \multicolumn{4}{l}{ As-synthesized tetragonal PBCO } & \multicolumn{4}{l}{ As synthesized orthorhombic PBCO } \\
\hline & $\mathrm{a} / \AA$ & $\mathrm{c} / \AA$ & $\mathrm{a} / \AA$ & $\mathrm{b} / \AA$ & $\mathrm{c} / \AA$ \\
25 & 3.909 & 7.636 & 3.903 & 7.866 & 7.598 \\
500 & 3.943 & 7.717 & 3.938 & 7.932 & 7.704 \\
550 & 3.949 & 7.724 & 3.952 & & 7.712 \\
600 & 3.954 & 7.73 & 3.958 & & 7.72 \\
650 & 3.96 & 7.737 & 3.963 & & 7.725 \\
700 & 3.965 & 7.744 & 3.968 & & 7.732 \\
\hline
\end{tabular}

\subsection{Lattice Oxygen Vacancy Order/Disorder Transition}

Oxygen ordering degree is a critical factor in determining the electronic, magnetic, and structural properties of perovskites. For $\mathrm{LnBaCo}_{2} \mathrm{O}_{5}\left(\mathrm{Ln}=\right.$ rare earth) and $\mathrm{LnBaCo}_{2} \mathrm{O}_{5.5}$ two particular compositions, an ordering of oxygen vacancies possibly exists ${ }^{66}$. In $\mathrm{PrBaCo}_{2} \mathrm{O}_{5}$ the pyramidal positions are occupied by both $\mathrm{Co}^{3+}$ and $\mathrm{Co}^{2+}$ ions, which are present in an equal amount. In contrast, in $\mathrm{PrBaCo}_{2} \mathrm{O}_{5.5}$ only $\mathrm{Co}^{3+}$ ions occupied in alternating octahedral $\mathrm{CoO}_{6}$ and $\mathrm{CoO}_{5}$ domains. With this knowledge, the structure of alleged tetragonal and orthorhombic PBCO were further studied and characterized by neutron powder diffraction and transmission electron microscope.

For the PBCO sample treated in air, the room temperature NPD data (Figure 6.2) can be quite well refined using an average structure with lattice parameters $a \approx b \approx a_{\mathrm{p}}$ and $c \approx 2 a_{\mathrm{p}}$ (where $a_{\mathrm{p}}$ is the perovskite cubic cell) and $P 4 / \mathrm{mmm}$ space group. But when temperature increase to 600 ${ }^{\circ} \mathrm{C}$, there are additional small peaks present, which is attributed to a particular order of oxygen vacancies in the structure and can be indexed by doubling of both a and b parameters (so conserving the tetragonal symmetry). 

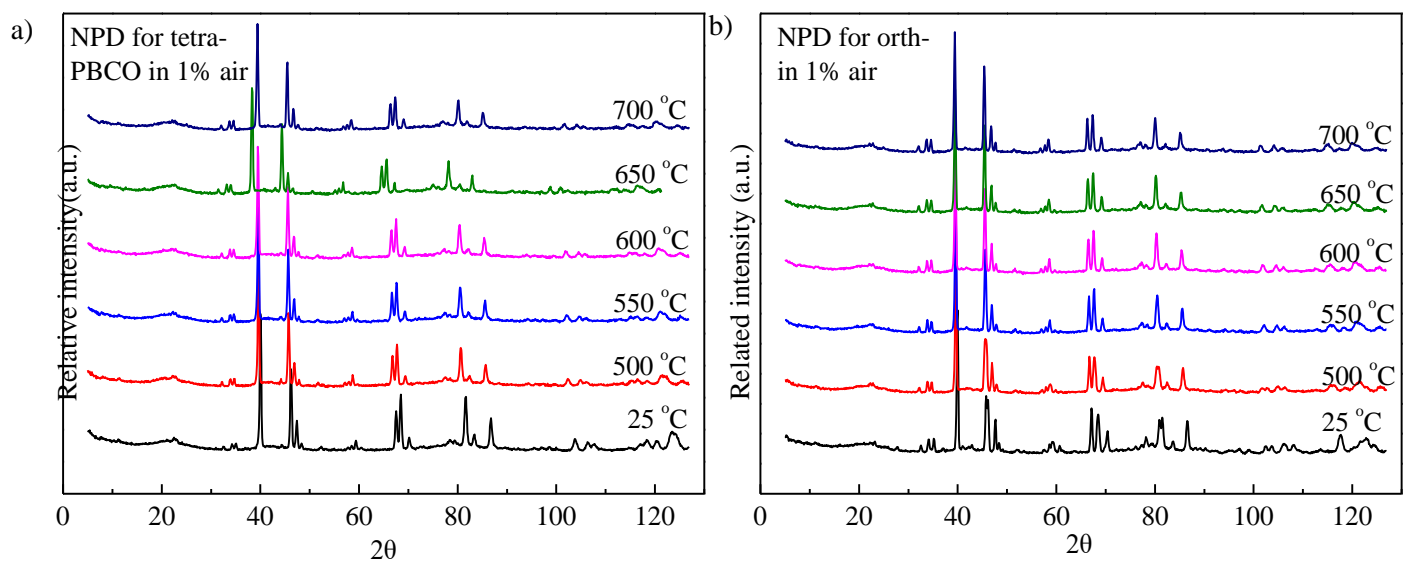

Figure 6.2 Neutron Powder diffraction for two as-synthesized PBCO in 1\% air.

The joint refinement of these two data sets for as-synthesized tetragonal PBCO has permitted the exploration of different possible ordered arrangements of the oxygen vacancies within the $\left[\mathrm{PrO}_{\delta}\right]$ planes. The first possibility corresponds to perfect order in a $2 a_{\mathrm{p}} \times 2 a_{\mathrm{p}}$ supercell in $\left[\mathrm{PrO}_{\delta}\right]$ planes, with vacancies located in $1 \mathrm{~b}\left[\left(\begin{array}{lll}0 & 0 & 1 / 2\end{array}\right)\right]$ Wyckoff position of the $P 4 / \mathrm{mmm}$. The second model was refined to represent the location of the vacancies in $\left(\begin{array}{lll}1 & 0 & 0\end{array}\right)$ lines, similarly to the order in $\mathrm{PBCO}_{5.5}$ layered cobaltites. Here, due to the smaller concentration of vacancies, these lines would be partially occupied forming a solid solution with oxygen ions. However, it violates the fourfold symmetry of the tetragonal $P 4 / \mathrm{mmm}$. The third possibility describes an imperfect order of the $2 a_{\mathrm{p}} \times 2 a_{\mathrm{p}}$ structure, in which oxygen vacancies are inhomogeneously distributed between $1 \mathrm{~b}\left[\left(\begin{array}{lll}0 & 0 & 1 / 2\end{array}\right)\right]$ and $1 \mathrm{~d}\left[\left(\begin{array}{lll}1 / 2 & 1 / 2 & 1 / 2\end{array}\right)\right]$ Wyckoff positions of the $P 4 / m m m$. The third scheme provides the best agreement with the peak intensities shown in Figure 6.3. Briefly, the refinement converges to the $25 \%$ of vacancies located in the $1 \mathrm{~d}$ position and the remaining $75 \%$ in the $1 \mathrm{~b}$ position. 

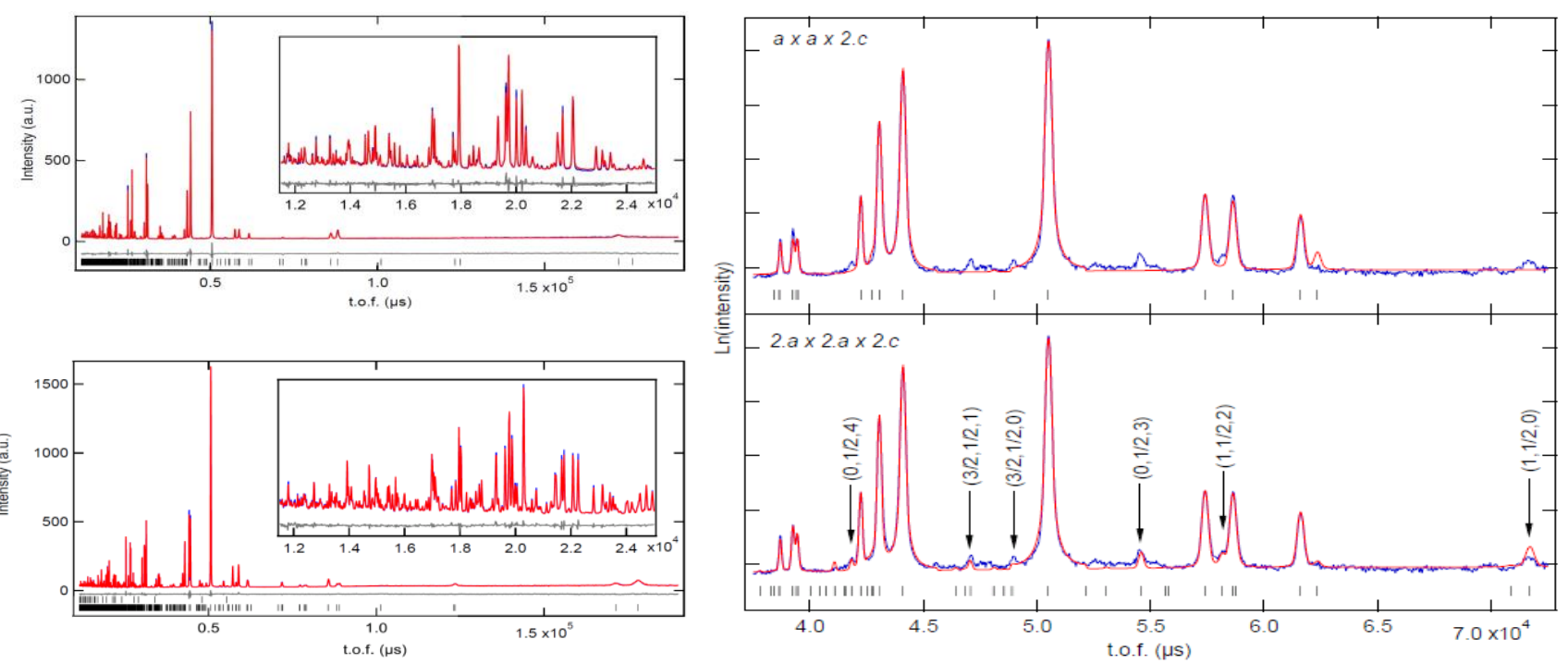

Figure 6.3 Room temperature neutron powder diffraction with TOF and fitting.

While NPD data for PBCO sample treated in nitrogen, the room temperature NPD data is labeled to an average structure with lattice parameters $\left(a \approx a_{\mathrm{p}}, b \approx 2 a_{\mathrm{p}}, c \approx 2 a_{\mathrm{p}}\right)$ and belongs to the Pmmm space group (conserving the orthorhombic symmetry). For PBCO with room temperature, the orthorhombic structure becomes tetragonal PBCO when above $600{ }^{\circ} \mathrm{C}$. Further, small peaks indexing to $2 a_{\mathrm{p}} \times 2 a_{\mathrm{p}} \times 2 a_{\mathrm{p}}$ which goes to the Pmma space group structure that appeared when above $650{ }^{\circ} \mathrm{C}$. Meanwhile, oxygen vacancies experience a transition from $100 \%$ order degree to ordered/disordered state in tetragonal structure and finally to a high disordered degree in a mixture of Pmma and Pmmm orthorhombic structure.

\subsection{Electron Microscopy Investigations of PBCO}

As shown from the Rietveld refinements of the NPD data, the structure of $\mathrm{PrBaCo}_{2} \mathrm{O}_{5.5}$ synthesized in nitrogen atmosphere deviates from the ideal structure by a partial disorder of the oxygen atoms at the level of the $\mathrm{PrO}_{0.5}$ layers. There is indeed observation of about $90 \%$ and $10 \%$ of the $\mathrm{O} 1$ and $\mathrm{O} 2$ sites by oxygen, respectively, instead of $100 \%$ and $0 \%$. Thus, TEM 
characterization is utilized here to investigate the $\mathrm{PrBaCo}_{2} \mathrm{O}_{5.5}$ sample to identify possible secondary phases, superstructures, and or particular microstructural features ${ }^{198}$. The TEM investigations performed on the PBCO wafer synthesized in air and nitrogen confirms their structures, tetragonal and orthorhombic, respectively.

In Figure 6.4, the selected area electron diffraction patterns of the $\mathrm{PrBaCo}_{2} \mathrm{O}_{5.5}$ synthesized in nitrogen confirms the observed spots can be indexed with Pmmm $a_{p} \times 2 a_{p} \times 2 a_{p}$ refined from the neutron powder diffraction. However, two additional features are observed. First, the investigated crystal grains present two domains $\mathrm{D}_{1}$ and $\mathrm{D}_{2}$ at a microscale level corresponding to the Pmmm $a_{p} \times 2 a_{p} \times 2 a_{p}$. The two domains are oriented $90^{\circ}$ with respect to each other (permutation of $a$ and $b$ axis). The zone axis patterns series shown in Figure 6.4a-b represent the typical double perovskite diffraction spots. The second type of rare seen [210] $]_{\mathrm{I}}$ or $\langle 110\rangle_{\mathrm{p}}$ zone axis pattern is presented in Figure 6.4c, and where some extra weak reflections could be indexed with another orientation ( $\mathrm{D}_{\text {III }}$ ) having the Pmmm $a_{p} \times 2 a_{p} \times 2 a_{p}$ structure. Second, in few areas of some investigated crystal grains, very weak extra reflections that cannot be indexed as Pmmm $a_{p} \times 2 a_{p} \times 2 a_{p}$ the structure is observed, as illustrated in Figure 6.2d. This indicates that these crystals possess a locally different structure from the 112-type cobaltite. The information available by SAED about such superstructures is blurred due to the superposition with the reflections coming from the Pmmm $a_{p} \times 2 a_{p} \times 2 a_{p}$ domains. Nonetheless, from the position of these extra spots, common characteristics can be summed up and imply, notably, the doubling of one of the $a_{p}$ parameter together with a centering of the cell. A part of these extra spots can be indexed considering the Pmma $2 a_{p} \times 2 a_{p} \times 2 a_{p}$ cell corresponding to the $\mathrm{LaBaMn}_{2} \mathrm{O}_{5.5}$ compound ${ }^{199}$. This is in agreement with the NPD results where the occupancies obtained for the $\mathrm{O} 1$ and $\mathrm{O} 2$ atomic positions can be locally attributed to a different vacancy/oxygen ordering 
leading notably to the existence of a faulted zones having a centered structural (122 type II). This tendency is evidenced by SAED but also visible in HREM for instance looking at one of the $\langle 110\rangle_{\mathrm{p}}$ directions, as shown in Figure 6.4e. The Pmmm $a_{p} \times 2 a_{p} \times 2 a_{p}$ structure is established all over the viewing area but locally in the area encircled the structure is clearly different and presents a centering also evidenced in the Fourier transform. 

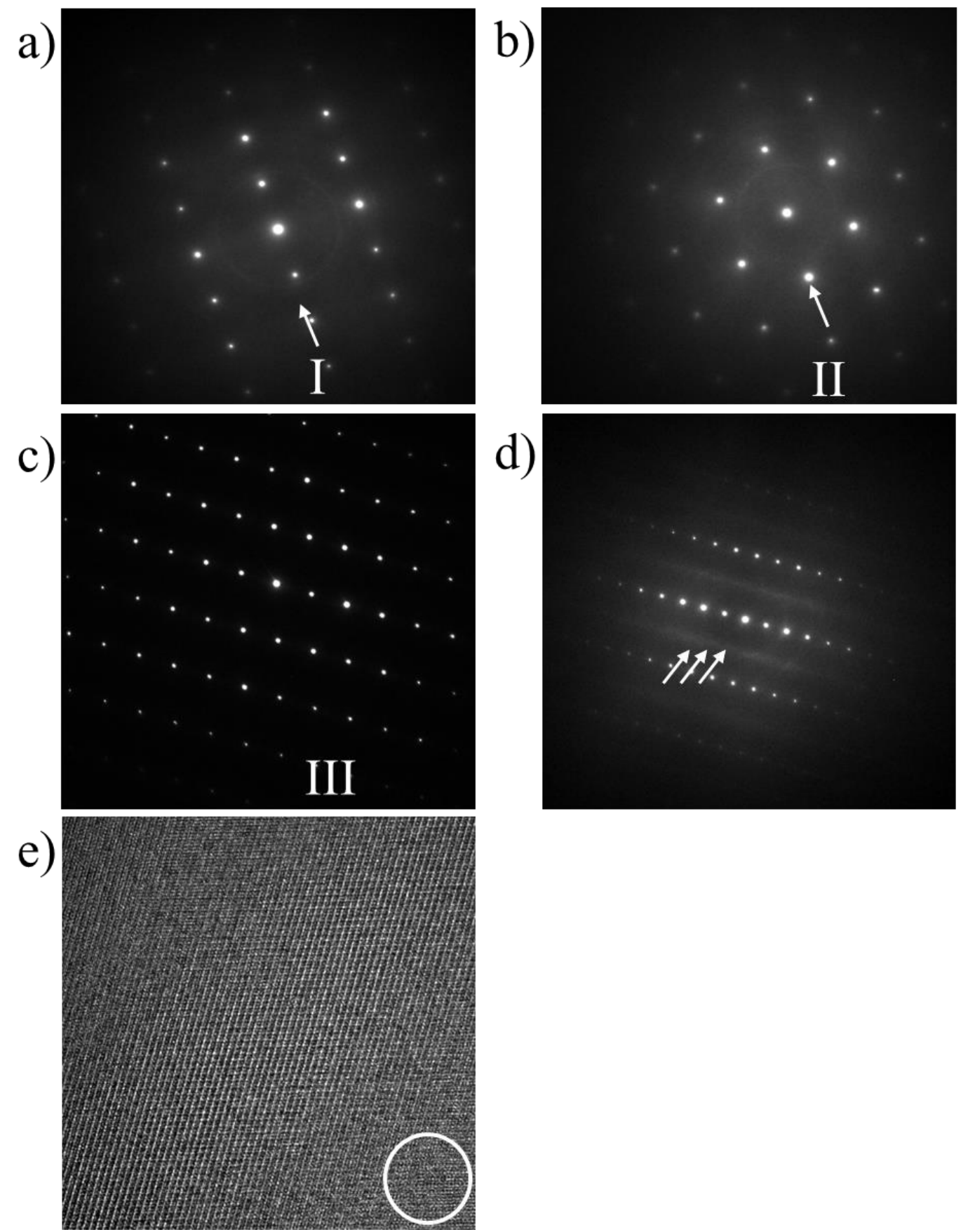

Figure 6.4 SAED patterns observed for $\mathrm{PrBaCo}_{2} \mathrm{O}_{5.5}$. The [100] zone axis patterns are displayed in (a) and (b) with two different diffraction domains I and II. Oriented domains ( $\mathrm{D}_{\mathrm{III}}$ ) can be revealed on the zone axis [210] in (c). Supplementary weak spots can be observed in (d). e) HREM image. 
Figure 6.5 illustrates the High-Resolution Electron Microscopy (HREM) images and the corresponding SAED patterns obtained along the [100] direction for the ordered layered $\mathrm{PrBaCo}_{2} \mathrm{O}_{5.5}$ material. The 122-type superstructure is clearly identified in both the HREM images and in the SAED patterns, by the doubling of the cell parameters along the [001] and [010] direction as compared to the disordered $\mathrm{La}_{0.5} \mathrm{Ba}_{0.5} \mathrm{CoO}_{3-\delta}$, where only a simple perovskite can be observed along any of the equivalent $\langle 100\rangle_{\mathrm{p}}$ zone axis directions.
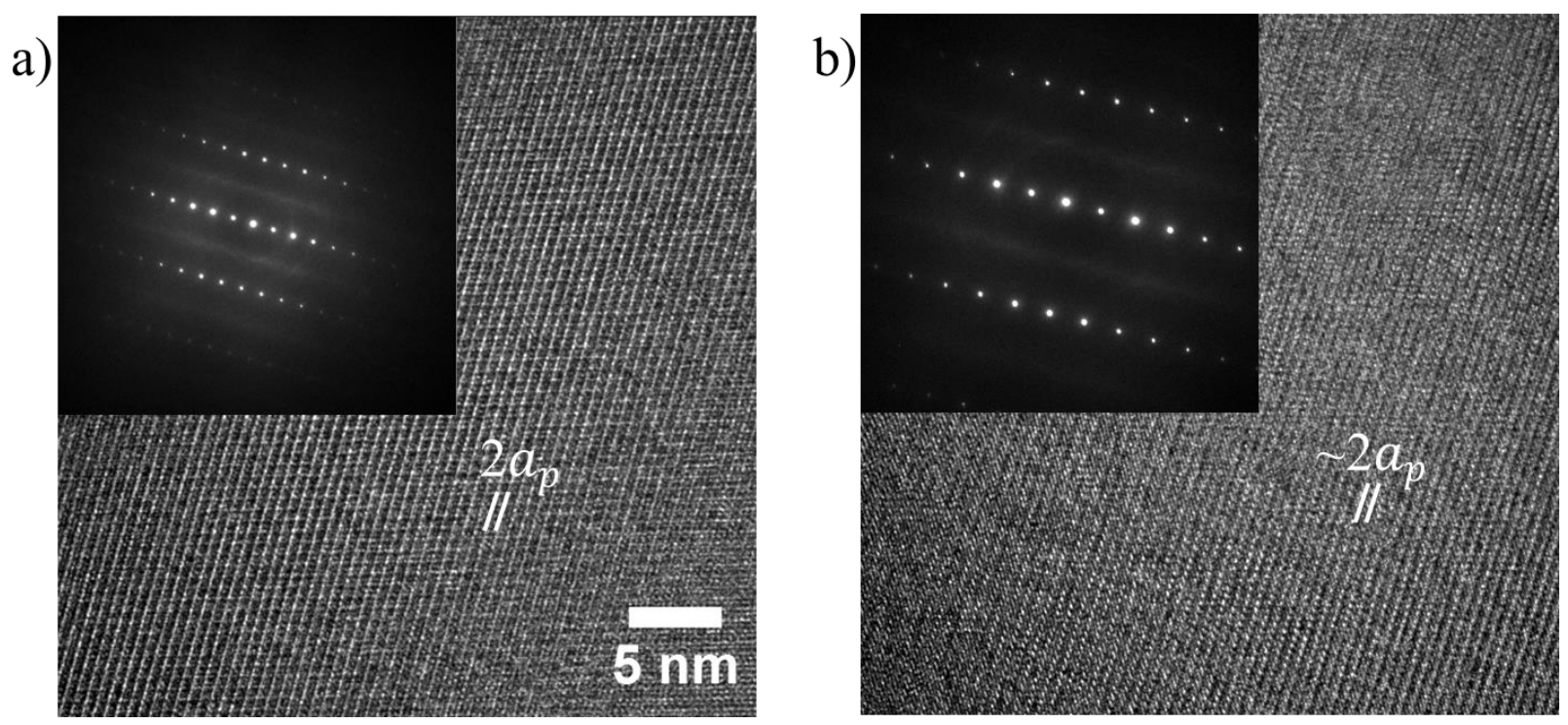

Figure 6.5 Typical HREM images and the corresponding SAED patterns obtained along the [100] direction.

The PBCO synthesized in air, as characterized by XRD and NPD can be indexed using a tetragonal P4mmm. However, the electron diffraction patterns of the tetragonal $\mathrm{LnBaCo}_{2} \mathrm{O}_{6-\delta}$ ( $\mathrm{Ln}=\mathrm{Nd}, \mathrm{Sm}, \mathrm{Eu}, \mathrm{Gd}, \mathrm{Tb}, \mathrm{Dy}$, and Ho) show the existence of superstructures without conditions limiting the reflections. Two kinds of supercells are observed according to the size of the lanthanide. For larger lanthanides, for example from $\mathrm{Nd}$ to $\mathrm{Tb}{ }^{200-202}$, corresponding to an $a_{p} \times 2 a_{p} \times 2 a_{p}$ supercell, whereas for smaller lanthanides, for $\mathrm{Ho}^{83}$ and Dy ${ }^{203}$, a tripling of 
two parameters is obtained, leading to a $3 a_{p} \times 3 a_{p} \times 2 a_{p}$ (similar to that reported in $\mathrm{YBaCo}_{2} \mathrm{O}_{5}$ annealed in pure oxygen ${ }^{204}$. A variation of oxygen content and of the structure with the size of the lanthanide suggests a possible ordering between oxygen and vacancies in the lanthanide layer. From the results reported for $\mathrm{YBaCo}_{2} \mathrm{O}_{5}{ }^{204}$, it clearly appears that the ordering of the oxygen vacancies in the perovskite matrix is responsible for the various superstructures observed in the electron diffraction ED patterns.

To make clear the sublattice of the PBCO synthesized in air and figure out its oxygen order degree status, the SAED experiment was also carried out on a PBCO wafer fabricated in air. One principal pattern viewed down the [001] directions is shown in Figure 6.6a and $a_{p} \times a_{p} \times 2 a_{p}$ the superstructure was observed. High-resolution TEM may be of great interest to obtain information about the size of the 112-type domains. Despite the domain size labeled in Figure 6.6d, dislocations and strain fields were found in the HREM image, as illustrated in Figure 6.6b. Clear grain boundaries were discovered with different crystal structures, as shown in Figures 6.6c and 6.6d. Lattice distortions were also found in Figure 6.6e. So, PBCO synthesized in the air should have a larger degree of disorder than that of sintered in nitrogen. 
a)

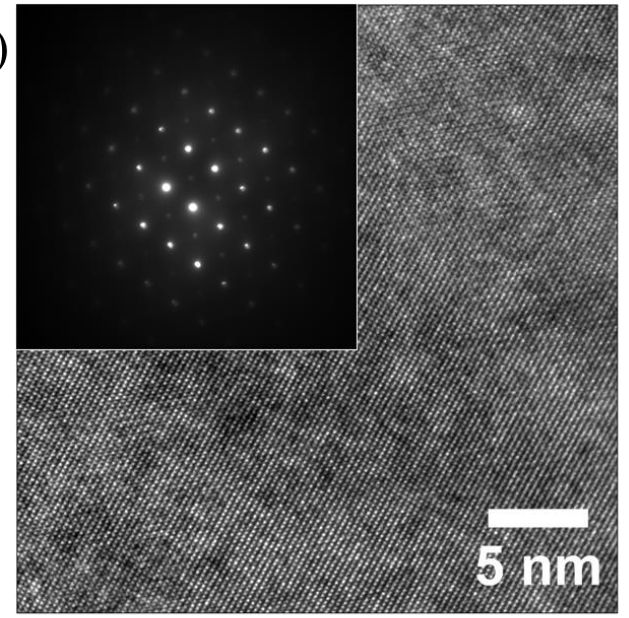

c)
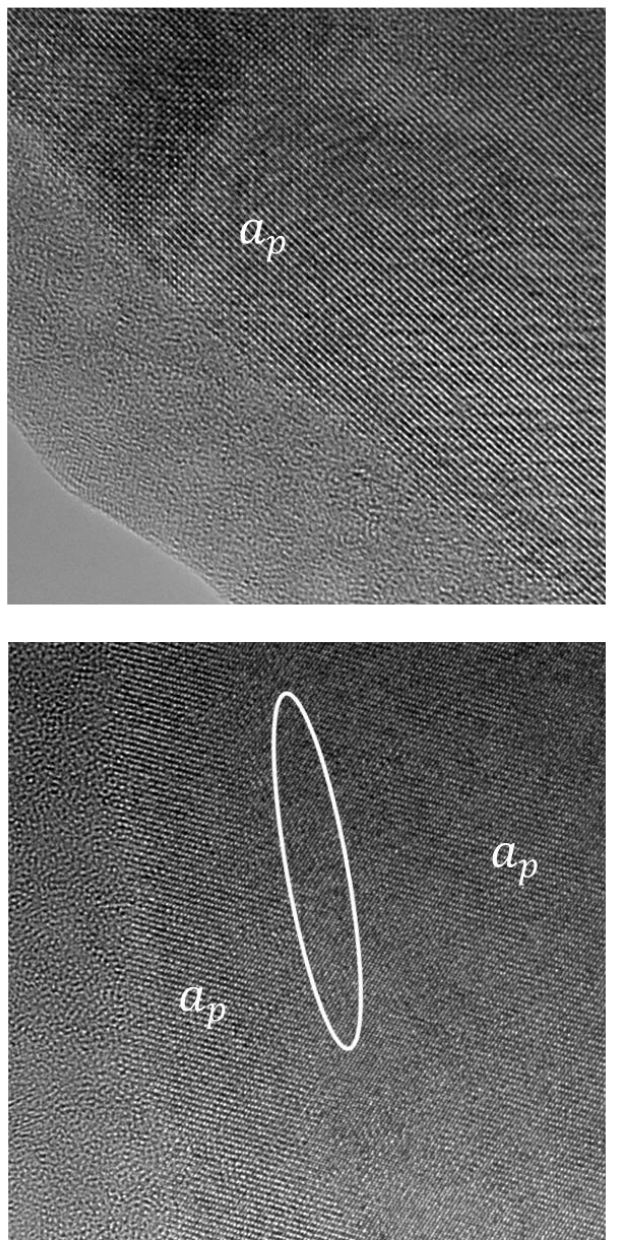

b)

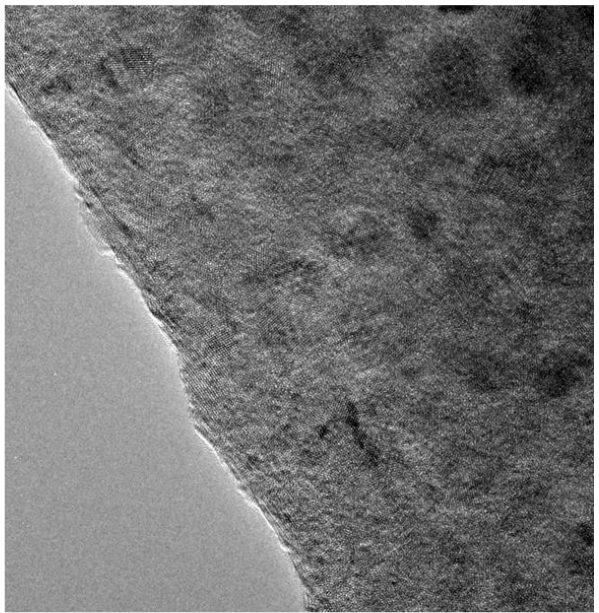

d)

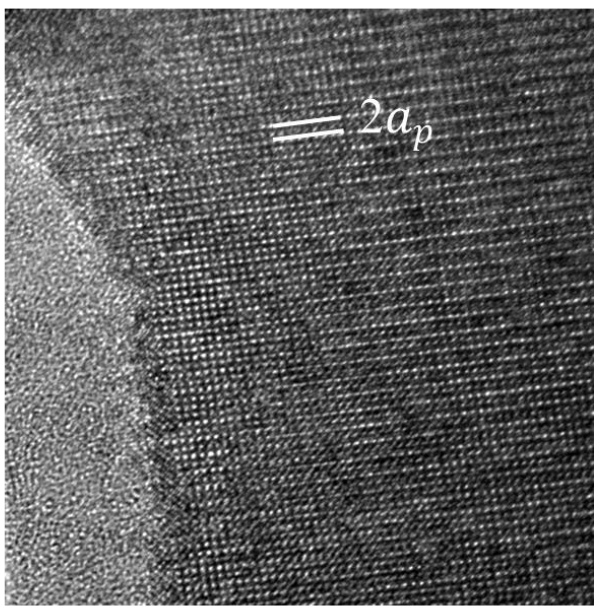

Figure 6.6 SAED patterns obtained along the [100] direction in a). b), c), and d) HREM of PBCO calcined in air. 


\subsection{Oxygen Non-stoichiometry Determined by TGA}

In solid-state ionic materials, the defect is a critical factor in determining electro-catalytic performance. Usually, high oxygen vacancy concentration will promote high oxygen ionic conductivity when hopping energy between defects is determined. To determine the oxygen vacancy concentration, oxygen non-stoichiometry needs to be confirmed first. The room temperature oxygen non-stoichiometry values in $\mathrm{PrBaCo}_{2} \mathrm{O}_{6-\delta}$ were 0.32 and 0.5 for the synthesized tetragonal and synthesized orthorhombic PBCO, respectively determined by iodine titration. The weight loss during temperature increase was determined by TGA. Figure 6.7 shows the weight change of pristine tetragonal in different oxygen atmospheres during heat up to 700 ${ }^{\circ} \mathrm{C}$. The first weight-loss period before oxygen uptake is mainly the water desorption process. Oxygen uptake between $200{ }^{\circ} \mathrm{C}$ and $300{ }^{\circ} \mathrm{C}$ is synchronized with cobalt oxidation from $\mathrm{Co}^{3+}$ to $\mathrm{Co}^{4+}$, this even occurred in $1 \%$ oxygen. For orthorhombic PBCO which experience phase transition, there's more oxygen uptake compared to tetragonal PBCO when in the same oxygen content gas.
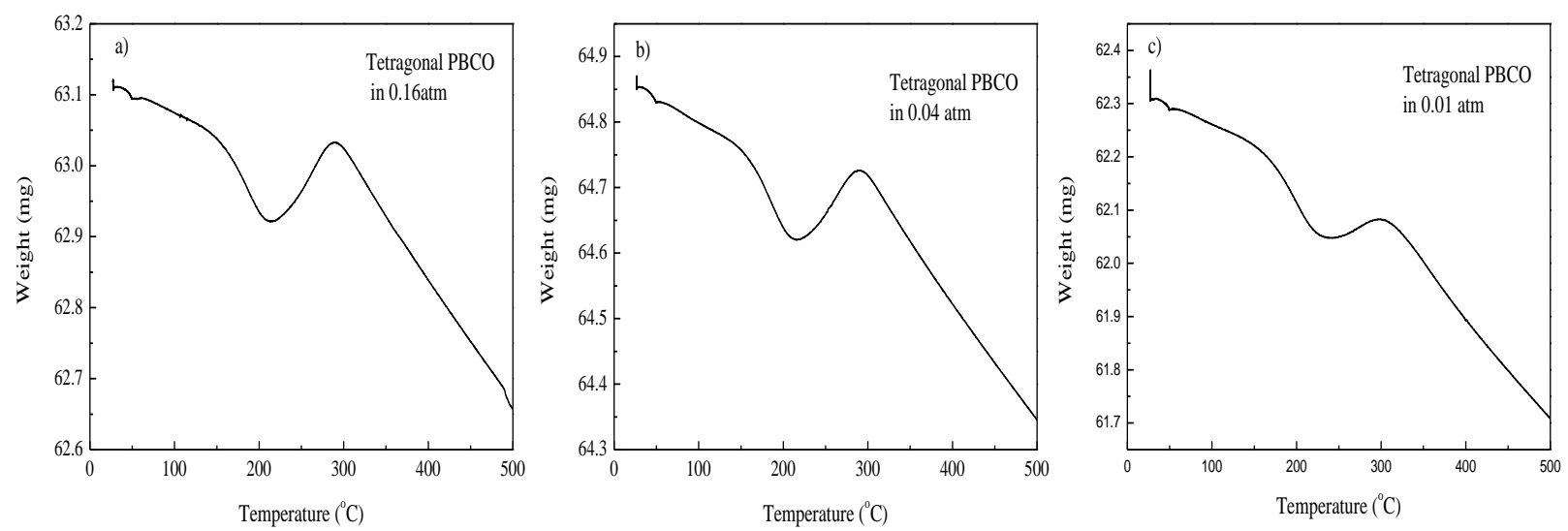

Figure 6.7 Weight loss of PBCO in different oxygen atmospheres below $500{ }^{\circ} \mathrm{C}$. 
The calculated $\delta$ values are shown in Figure 6.8. It shows that tetragonal PBCO will lose more oxygen than orthorhombic PBCO. The oxygen atom loss gap is around 0.11 except in $16 \%$ oxygen content gas atmosphere with oxygen loss of pristine orthorhombic PBCO even larger than that of tetragonal PBCO. However, oxygen non-stoichiometry of tetragonal PBCO is still less than that of orthorhombic PBCO with 6 oxygen atoms in the formula as a baseline. But it does not mean that oxygen transport in orthorhombic PBCO will be faster than that in tetragonal PBCO considering the mobility of oxygen has not been studied here yet.

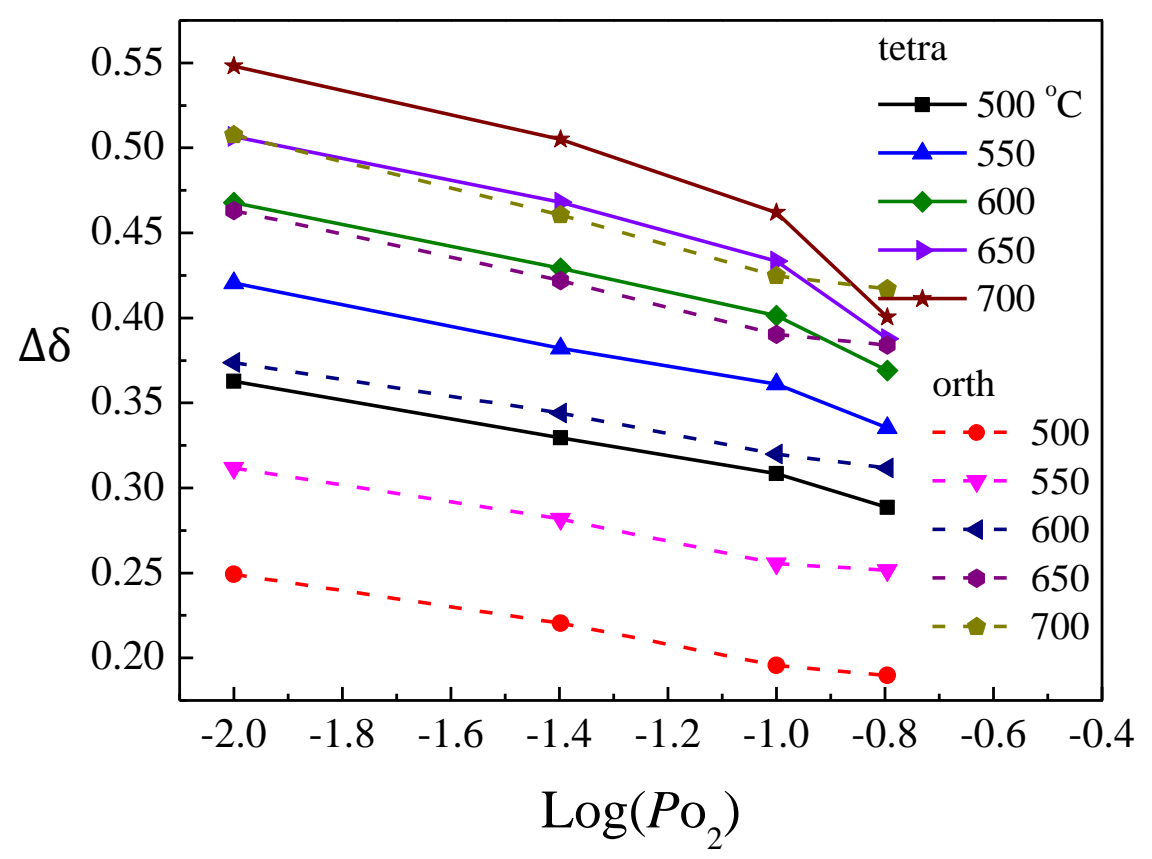

Figure 6.8 Oxygen non-stoichiometry values obtained from TG results.

The thermodynamic properties of PBCO with two different initial structure needs to be compared to further investigate the mobility of them. From the thermodynamic factor calculation formula (6-1), the relationship between oxygen non-stoichiometry and oxygen partial pressure was derived and shown in formula (6-2). 


$$
\begin{gathered}
f=\frac{1}{2} \frac{\partial \ln \left(P_{\mathrm{O}_{2}}\right)}{\partial \ln (6-\delta)} \\
\delta=6-\left(6-\delta_{0}\right) \exp \int_{\ln \left(P_{\mathrm{O}_{2}}^{0}\right)}^{\ln \left(P_{\mathrm{O}_{2}}\right)} \frac{1}{2 f_{\left(\ln \left(P_{\mathrm{O}_{2}}\right)\right)}} d \ln P_{\mathrm{O}_{2}}
\end{gathered}
$$

The chemical potential, $\mu_{\mathrm{O}}$, of $\mathrm{PrBaCo}_{2} \mathrm{O}_{6-\delta}$ is expressed in formula (6-3):

$$
\mu_{\mathrm{O}}-\mu_{\mathrm{O}}^{\circ}=\frac{R T}{2} \ln \left(P_{\mathrm{O}_{2}}\right)
$$

Where superscript zero denotes equilibrium with 1 bar oxygen. The oxygen chemical potential is written with the partial molar enthalpy and partial molar entropy of oxygen expressed in the following formulas:

$$
\begin{gathered}
\mu_{\mathrm{O}}-\mu_{\mathrm{O}}^{\circ}=\left(h_{\mathrm{O}}-h_{\mathrm{O}}^{\circ}\right)-T\left(s_{\mathrm{O}}-s_{\mathrm{O}}^{\circ}\right) \\
h_{\mathrm{O}}-h_{\mathrm{O}}^{0}=\frac{\partial}{\partial\left(\frac{1}{T}\right)}\left(\frac{R}{2} \ln P_{\mathrm{O}_{2}}\right) \\
s_{\mathrm{O}}-s_{\mathrm{O}}^{0}=-\frac{\partial}{\partial T}\left(\frac{R T}{2} \ln P_{\mathrm{O}_{2}}\right)
\end{gathered}
$$

Figure 6.9 and Figure 6.10 exhibit $R / 2 \ln P\left(\mathrm{O}_{2}\right)$ vs. $1 / T$ plots and $R T \ln P\left(\mathrm{O}_{2}\right)$ vs. $T$ plots of $\mathrm{PrBaCo}_{2} \mathrm{O}_{5.68-\delta}$ and $\mathrm{PrBaCo}_{2} \mathrm{O}_{5.5-\delta}$ respectively for selected $\delta$. There are inflection points for both PBCO structures which correspond to the influence of the structure evolution on the performance of the materials. As-synthesized tetragonal PBCO shows abrupt change at $600{ }^{\circ} \mathrm{C}$ in all stoichiometric oxygen content listed here. This phenomenon corresponds to the non-linear relationship between the bulk diffusion coefficient and temperature. For pristine orthorhombic PBCO, this phenomenon occurred at $650{ }^{\circ} \mathrm{C}$. These blunt changes of thermodynamic quantites that structural evolution exists in these materials. Another portent is that the value of $h_{\mathrm{O}}-h_{\mathrm{O}}^{0}$ and $s_{\mathrm{O}}-s_{\mathrm{O}}^{0}$ for tetragonal PBCO almost approaches zero as $\delta$ close to 0.5 . The oxygen partial 
molar enthalpy increases when there is more oxygen loss for both the as-synthesized tetragonal and the pristine orthorhombic PBCO during the structure evolution with temperature and partial pressure, which easily explains why there are more oxygen vacancies formed when there is oxygen loss in the structure. The as-synthesized tetragonal PBCO has higher partial molar enthalpy than the pristine orthorhombic $\mathrm{PBCO}$ during structure evolution, meaning that it is more likely to form more oxygen vacancy for the former structure than the latter. However, the oxygen vacancy formation enthalpy of the pristine orthorhombic $\mathrm{PBCO}$ is smaller than the assynthesized tetragonal PBCO during structure evolution. So, the pristine tetragonal PBCO easily

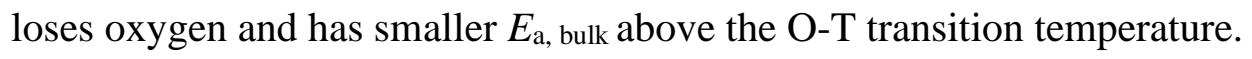
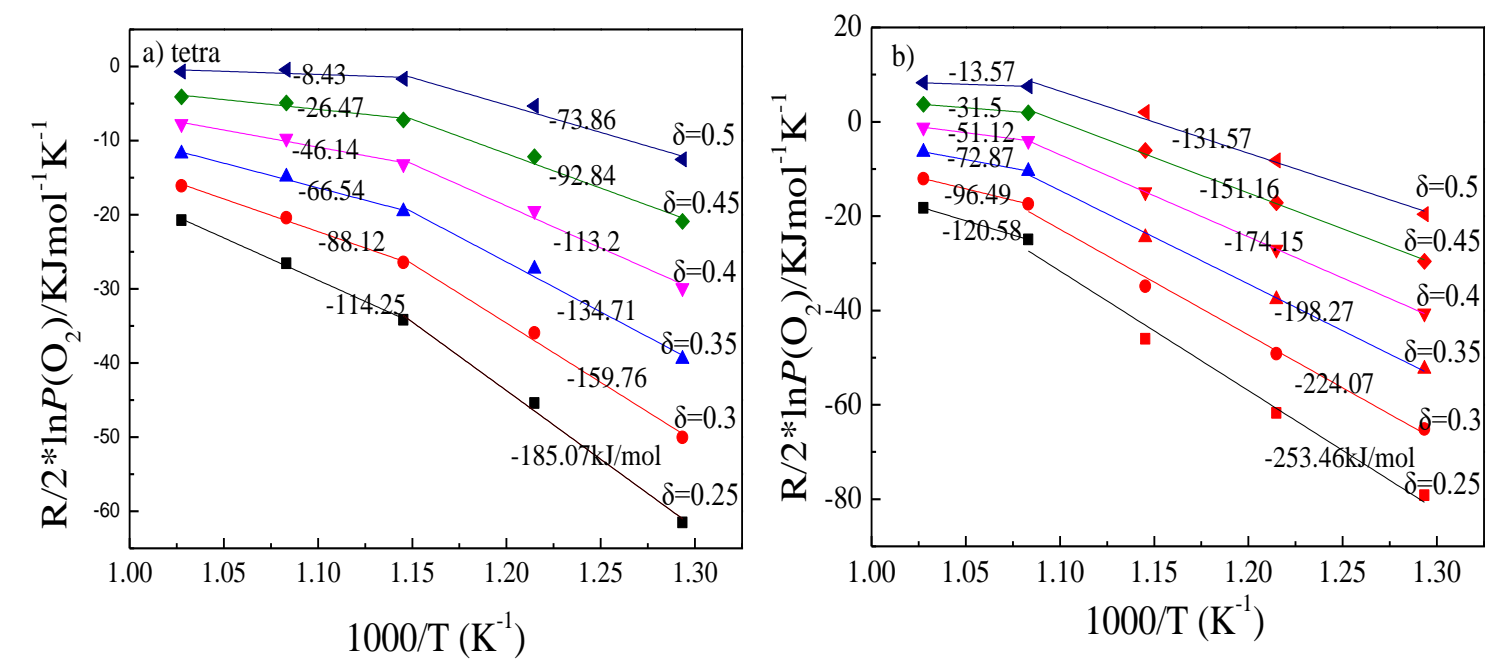

Figure 6.9 Relation between $\frac{R}{2} \ln P_{\mathrm{O}_{2}}$ and $\frac{1}{T}$ with $\delta$ as a parameter: a) tetra- PBCO, b) orth- PBCO. From the slope of the plots, $\Delta H_{\mathrm{O}}$ is calculated.

With the increase in $\delta$, the average valence of cobalt ions decreases. But oxygen vacancy concentration does not always increase with $\delta$, since $\Delta H_{0}$ do not have a linear relationship with $\delta$. The binding energy of oxygen ions decreases with an increase in $\delta$, but not linearly. When the non-stoichiometry oxygen vacancies are considered pseudo-chemical species and the oxygen sublattice as a solution of oxygen vacancies and oxygen ions, the solution can be expressed as a 
regular solution. However, the non-linear relationship between the partial molar quantities and $\delta$ implies the non-ideal solution of oxygen vacancies and oxygen ions ${ }^{205-208}$. For the non-ideal solution here, oxygen vacancy will associate with the antisite defect $B a_{P r}^{\prime}$ and become immoblized.
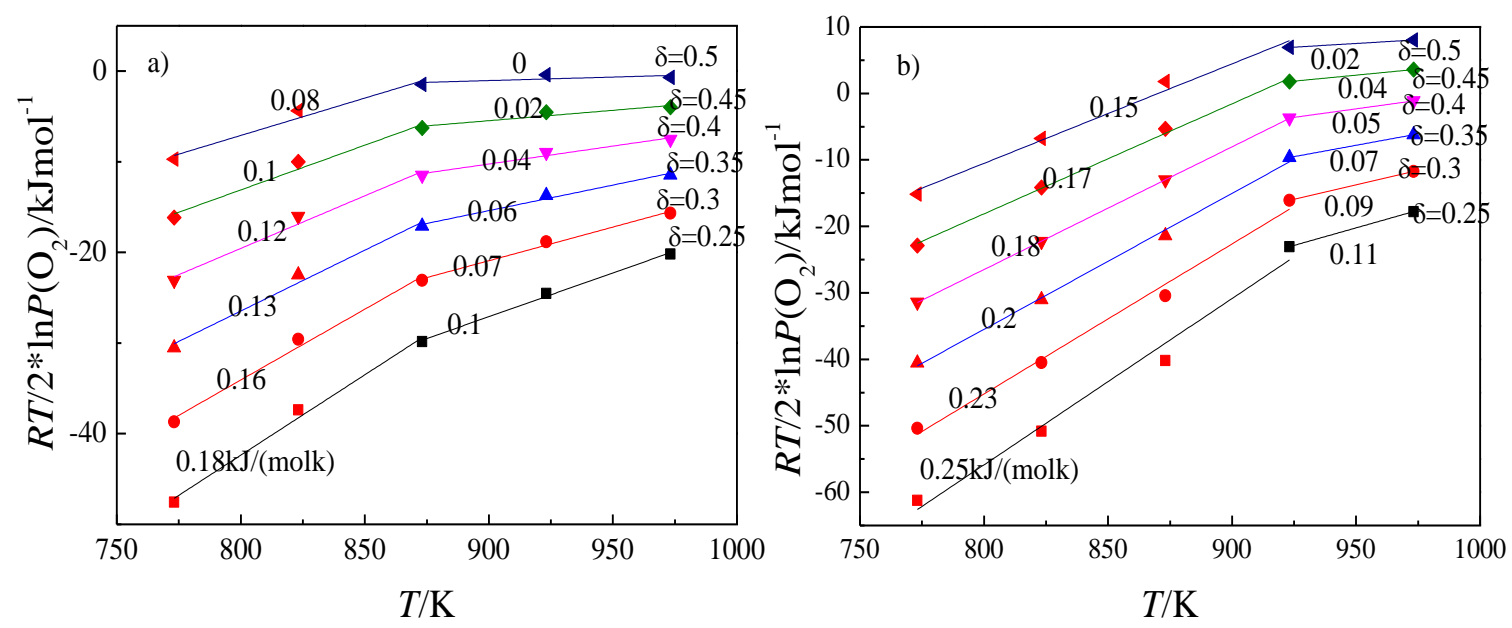

Figure 6.10 Relation between $\frac{R T}{2} \ln P_{\mathrm{O}_{2}}$ and $T$ with $\delta$ as a parameter: a) tetra $\mathrm{PBCO}, \mathrm{b}$ ) orth PBCO. From the slope of the plots, $\Delta S_{\mathrm{O}}$ is calculated.

\subsection{Summary}

In this chapter, the structures of PBCO treated in air and nitrogen were primarily characterized using X-ray powder diffraction. The result shows tetragonal and orthorhombic structures at room temperature respectively in the limit of the XRD instrument. The tetragonal structure keeps in operation range of SOFC, while orthorhombic transformed to tetragonal when above $550{ }^{\circ} \mathrm{C}$. The comparison of the lattice parameters refinement gives a possible explanation for the oxygen transport discrepancy since the fast transport bond length was shorter for tetragonal PBCO than orthorhombic PBCO with temperature increasing.

The room temperature NPD data of PBCO treated in the air can be well-matched with the $P 4 / \mathrm{mmm}$ space group. However, when the temperature goes above $600{ }^{\circ} \mathrm{C}$, a new crystal lattice 
appeared with a doubling of the $a$ and $b$ parameters. The tetragonal symmetry is conserved. Three possible oxygen vacancy order arrangement models were utilized to refine the NPD profile. The third model refines the NPD data well, which describes an imperfect order with oxygen vacancies inhomogeneously distributed between $\left.1 \mathrm{~b}\left[\begin{array}{lll}0 & 0 & 1 / 2\end{array}\right)\right]$ and $1 \mathrm{~d}\left[\left(\begin{array}{lll}1 / 2 & 1 / 2 & 1 / 2\end{array}\right)\right]$ Wyckoff positions of the $P 4 / \mathrm{mmm}$. The NPD data of PBCO treated in nitrogen verified the Pmmm space group with orthorhombic symmetry and oxygen homogeneously distributed between 1c [( $\left.\left.\begin{array}{lll}0 & 0 & 1 / 2\end{array}\right)\right]$ and $1 \mathrm{~g}\left[\left(\begin{array}{lll}0 & 1 / 2 & 1 / 2\end{array}\right)\right]$ Wyckoff positions with the occupancy of $10 \%$ at $1 \mathrm{c}$ and $90 \%$ at $1 \mathrm{~g}$.

The SAED patterns also prove the crystal structures refined from the NPD data of the PBCO wafer prepared in nitrogen. However, besides the cation-ordered tetragonal symmetry lattice, the crystal lattice with simple cubic symmetry was discovered in the PBCO wafer treated in the air. In addition, dislocations, strain fields, and lattice distortions were also found in the PBCO wafer synthesized in the air. The data confidently indicates that PBCO treated in the air has a higher disorder degree than that of treated in nitrogen.

The study of oxygen non-stoichiometry by the TGA method proves that there will be more oxygen vacancy formed for tetragonal PBCO with temperature increase. The thermodynamic quantities obtained through solving the thermodynamic factor equation show nonlinear relationships with temperature. This may be caused by a structure transformation or the appearance of a new phase, even the association of the defects. 


\section{Oxygen Transport Mechanism of Dense PBCO and ORR Analysis on Porous PBCO Cathode}

The studies of tetragonal PBCO used as SOFC cathode so far have shown their low polarization resistance which is $0.4 \Omega \cdot \mathrm{cm}^{2}$ at $600{ }^{\circ} \mathrm{C}, 0.21 \mathrm{~atm}$ for polycrystalline material ${ }^{209}$. In chapter 5 , the polarization resistance for tetragonal $\mathrm{PBCO}$ tested at $600{ }^{\circ} \mathrm{C}$ is $0.165 \Omega \cdot \mathrm{cm}^{2}$. In the thin-film SOFC cathode domain, single-crystalline thin films PBCO coated by pulsed laser deposition have been investigated to yield accurate oxygen surface exchange coefficient $k$. The lowest areaspecific resistance (ASR) reported for thin-film PBCO at $600{ }^{\circ} \mathrm{C}$ in the air is $0.11 \Omega \cdot \mathrm{cm}^{2}$, and the $k$ value is $6.0 \times 10^{-3} \mathrm{~cm} / \mathrm{s}$ at $600{ }^{\circ} \mathrm{C}{ }^{62}$. The high electrocatalytic performance for the oxygen reduction reaction $(\mathrm{ORR})$ on the tetragonal structures is mainly attributed to three reasons. They are the high oxygen vacancy concentration, the shorter M-O bond strength, and the anisotropic of oxygen transport between the ab plane and $\mathrm{c}$ axis ${ }^{12,62,115,210,211}$. However, the performance difference between the as-synthesized tetragonal and as-synthesized orthorhombic PBCO which have different order degrees has not been evaluated in the literature. Besides, the oxygen transport properties for polycrystalline porous cathode have not been made clear so far. The ORR on tetragonal PBCO cathode has been discussed generally in chapter 5. But, the ratedetermining steps have not been discussed in detail yet. The ORR process on the as-synthesized orthorhombic also needs to be investigated to further explain the performance present. Although ORR involves several elementary steps: gas diffusion, adsorption or dissociative adsorption on the cathode, incorporation of oxygen into the lattice, bulk diffusion through the cathode, charge transfer at the gas/cathode or cathode/electrolyte interface, the reaction rate should be limited by one or more steps. 
To study the electrocatalytic performance and mechanism of both the tetragonal and orthorhombic PBCO cathode, the electrical conductivity relaxation method was adopted to investigate the oxygen transport properties of the materials. The relationship between the structure and the oxygen transport performance needs to be built to explain how the structure affects oxygen vacancy behavior which determines the oxygen bulk diffusion performance. The ORR mechanism then could be deeply probed with the oxygen vacancy formation and transport information.

\subsection{K and D Calculated from the Fitting of the ECR Curve}

Electrical conductivity relaxation (ECR) is one convenient and economic way to study oxygen transport kinetics including surface exchange and bulk diffusion processes. The fitting method introduced by Li et al was adopted here to reduce the errors caused by the determination of the initial point ${ }^{212}$. In the electrical conductivity relaxation experiment, a sintered dense pellet is used. The PBCO pellets are annealed at the chosen temperature and $P_{\mathrm{O}_{2}}$ to equilibrium stoichiometry. Afterward, a step-change in $P_{\mathrm{O}_{2}}$ is applied and the time-variant electrical conductivity of a sample is measured. To determine oxygen surface exchange $\left(k_{\mathrm{ex}}\right)$ and bulk diffusion coefficients $\left(D_{\text {chem }}\right)$, a correlation between a mean conductivity and the corresponding mean non-stoichiometry had been established ${ }^{213}$. Assuming that the mobility of electrons and holes are independent of the non-stoichiometry or oxygen partial pressure at a certain temperature, the following oxygen non-stoichiometry and total conductivity correlation can be derived:

$$
\frac{\delta_{t}-\delta_{0}}{\delta_{\infty}-\delta_{0}}=\frac{\sigma_{t}-\sigma_{0}}{\sigma_{\infty}-\sigma_{0}}
$$


Since the non-stoichiometry can stand for oxygen concentration inside the material, similar relation between oxygen concentration and conductivity can be formed:

$$
\frac{C_{t}-C_{0}}{C_{\infty}-C_{0}}=\frac{\sigma_{t}-\sigma_{0}}{\sigma_{\infty}-\sigma_{0}}
$$

Solid-phase equilibrium will be restored when the surface exchange is equal to the diffusion flux which is presented by the equation:

$$
-D \partial \mathrm{C} /\left.\partial \mathrm{x}\right|_{x= \pm a}=k\left[C_{(\infty)}-C_{(t)}\right]
$$

Based on the boundary conditions and Fick's second laws $\left(\frac{\partial C}{\partial t}=D \frac{\partial^{2} C}{\partial x^{2}}\right)$, The solution for a pellet sample is:

$$
\begin{gathered}
\frac{\sigma_{t}-\sigma_{0}}{\sigma_{\infty}-\sigma_{0}}=1-\sum_{n=1}^{\infty} \frac{2 L^{2} \exp \left(-b_{n}^{2} D t / a^{2}\right.}{b_{n}^{2}\left(b_{n}^{2}+L^{2}+L\right)} \\
L=\frac{a}{l_{c}}=\frac{a k}{D}=b_{n} \tan b_{n}
\end{gathered}
$$

Here, $a$ is the half-thickness of the tested pellet sample in the diffusion solution. And $L$ is a unit length parameter from which the characteristic thickness $l_{c}$ can be derived.

Figure 7.1a shows the fitting error color maps in the $D, k$ plane for the simultaneous analysis of the data set obtained. The appearance of the dark-blue region indicates the high accuracy of the fitting results. Figure 7.2 presents a visualized scheme that how quick it can be for oxygen to approach equilibrium with both pristine tetragonal and orthorhombic PBCO. Oxygen bulk chemical diffusion is more sensitive to oxygen partial pressure change for orthorhombic PBCO and more sensitive to temperature variation for tetragonal PBCO. Since the estimated diffusion coefficient $D$ is pretty high $\left(10^{-6} \mathrm{~cm}^{2} / \mathrm{s} \sim 10^{-4} \mathrm{~cm}^{2} / \mathrm{s}\right)$ compared to surface exchange coefficient $k$, 
it is difficult to fit the accurate $D$ values considering the dense and thickness requirement for samples. Thus, the fitting of $D, k$ values are on two different thickness PBCO (0.05 and $1.5 \mathrm{~mm})$. The fitted results are listed in Table 7.1 and Table 7.2. $L$ values calculated from $k, D$ tested in air and $1 \%$ oxygen at $500{ }^{\circ} \mathrm{C}, 550{ }^{\circ} \mathrm{C}, 600{ }^{\circ} \mathrm{C}, 650{ }^{\circ} \mathrm{C}$, and $700{ }^{\circ} \mathrm{C}$ are also listed in the Tables. Generally, if $L<0.1$, the oxygen transport process should be surface limited, if larger than 10 , it is bulk diffusion-controlled if $0.1<L<10$, it should be deemed as a mixed control process here. As a result, both the as-synthesized tetragonal and orthorhombic PBCO should be assumed as surface limited materials.

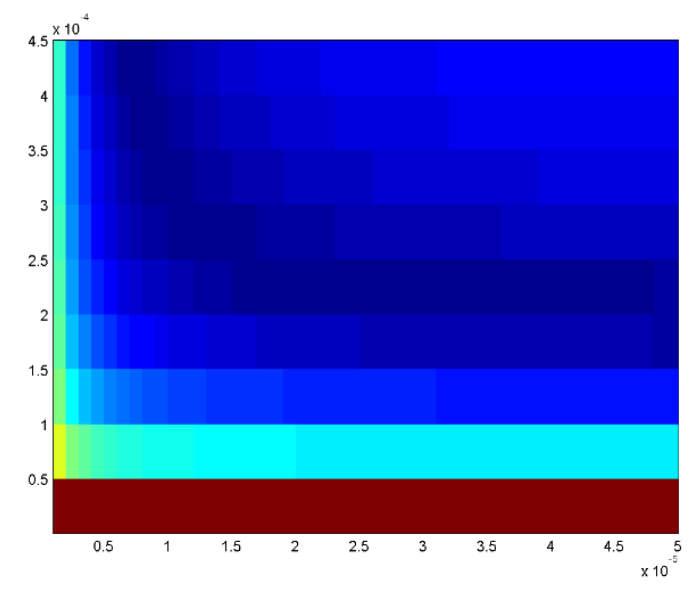

(a)

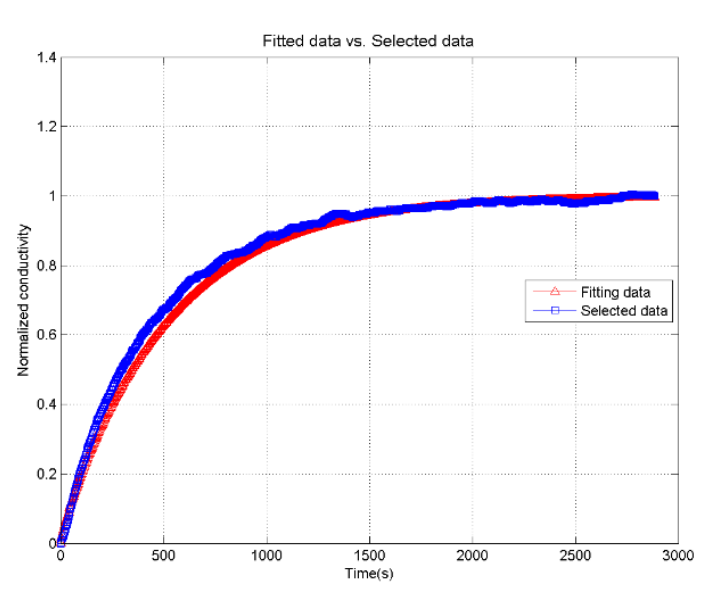

(b)

Figure 7.1 a) Error color map for fitting single noisy relaxation data Error map for a wide range of $k$ and D. b) Normalized conductivity and fitting curve.

The results show that the bulk diffusion coefficients and surface exchange coefficients are in the same order of magnitude, indicate the activation of the whole electrode if used as SOFC cathodes. In the air atmosphere, oxygen ion transport in tetragonal $\mathrm{PBCO}$ is slower than that in orthorhombic $\mathrm{PBCO}$ at $550{ }^{\circ} \mathrm{C}$. Besides, this phenomenon even appeared at $600{ }^{\circ} \mathrm{C}$ in a $1 \%$ air atmosphere. 

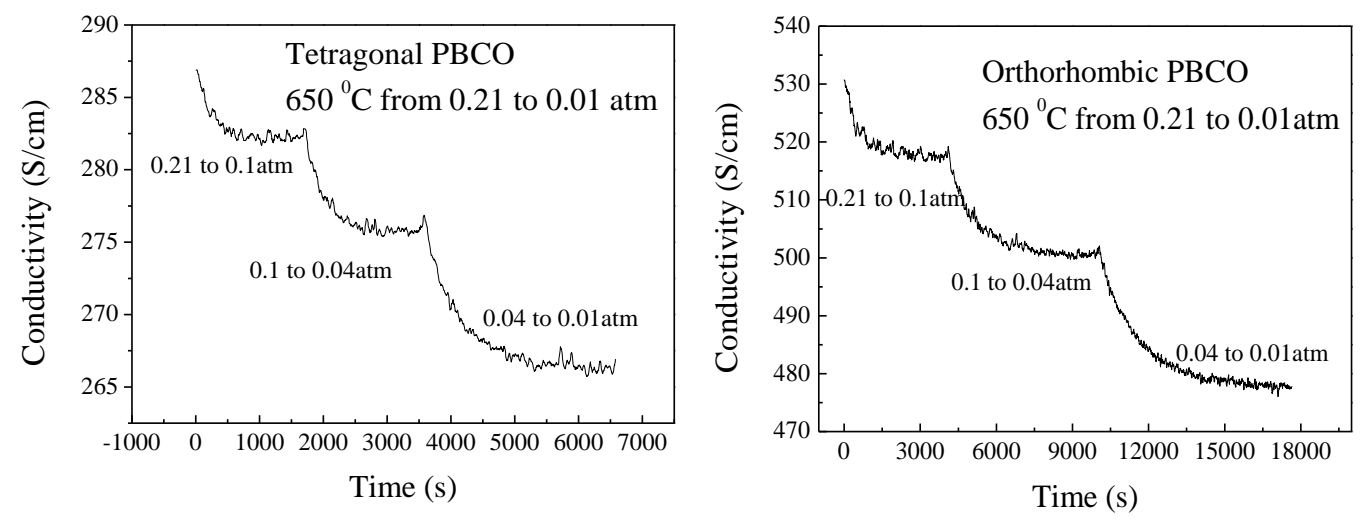

Figure 7.2 Electrical conductivity relaxation of pristine tetragonal and orthorhombic PBCO with time.

Table 7.1 oxygen transport parameters for as-synthesized tetragonal PBCO and as-synthesized PBCO in air.

\begin{tabular}{ccccccc}
\hline & \multicolumn{3}{c}{ As-synthesized tetra- PBCO } & \multicolumn{3}{c}{ As-synthesized ortho- PBCO } \\
& $k(\mathrm{~cm} / \mathrm{s})$ & $D\left(\mathrm{~cm}^{2} / \mathrm{s}\right)$ & $\mathrm{L}$ & $k(\mathrm{~cm} / \mathrm{s})$ & $D\left(\mathrm{~cm}^{2} / \mathrm{s}\right)$ & $\mathrm{L}$ \\
\hline $700^{\circ} \mathrm{C}$ & $2.30 \mathrm{E}-04$ & $2.40 \mathrm{E}-04$ & $4.79 \mathrm{E}-02$ & $1.28 \mathrm{E}-04$ & $1.40 \mathrm{E}-04$ & $4.57 \mathrm{E}-02$ \\
$650{ }^{\circ} \mathrm{C}$ & $1.36 \mathrm{E}-04$ & $1.30 \mathrm{E}-04$ & $5.23 \mathrm{E}-02$ & $9.70 \mathrm{E}-05$ & $1.10 \mathrm{E}-04$ & $4.41 \mathrm{E}-02$ \\
$600{ }^{\circ} \mathrm{C}$ & $9.01 \mathrm{E}-05$ & $8.50 \mathrm{E}-05$ & $5.30 \mathrm{E}-02$ & $6.81 \mathrm{E}-05$ & $6.20 \mathrm{E}-05$ & $5.49 \mathrm{E}-02$ \\
$550{ }^{\circ} \mathrm{C}$ & $4.23 \mathrm{E}-05$ & $2.50 \mathrm{E}-05$ & $8.46 \mathrm{E}-02$ & $5.23 \mathrm{E}-05$ & $3.20 \mathrm{E}-05$ & $8.17 \mathrm{E}-02$ \\
$500{ }^{\circ} \mathrm{C}$ & $2.70 \mathrm{E}-05$ & $7.50 \mathrm{E}-05$ & $1.80 \mathrm{E}-02$ & $3.20 \mathrm{E}-05$ & $1.20 \mathrm{E}-05$ & $1.33 \mathrm{E}-01$ \\
\hline
\end{tabular}

The oxygen surface exchange coefficient is much more complicated than bulk diffusion regarding the ambiguous surface state especially for polycrystalline. In simplification, oxygen bulk diffusion evolution with temperature and the gap between the two different original structures can be examined. The high oxygen bulk diffusion coefficient is an indicator of fast oxygen ion conduction, and one component of fast oxygen ion conduction is the low oxygen migration barrier. Optimized oxygen vacancy concentration and low oxygen migration barrier 
are typically the dominant factors leading to the fast bulk diffusion of oxygen in the lattice. Both two samples show high activity for oxygen activation and mobility.

Table 7. 2 oxygen transport parameters for as-synthesized tetragonal PBCO and as-synthesized PBCO in $1 \%$ oxygen.

\begin{tabular}{lllllll}
\hline \multicolumn{5}{c}{ As-synthesized tetra- PBCO } & \multicolumn{3}{c}{ As-synthesized ortho- PBCO } \\
& $k(\mathrm{~cm} / \mathrm{s})$ & $D\left(\mathrm{~cm}^{2} / \mathrm{s}\right)$ & $\mathrm{L}$ & $k(\mathrm{~cm} / \mathrm{s})$ & $D\left(\mathrm{~cm}^{2} / \mathrm{s}\right)$ & $\mathrm{L}$ \\
\hline $700^{\circ} \mathrm{C}$ & $1.08 \mathrm{E}-04$ & $1.00 \mathrm{E}-04$ & $5.40 \mathrm{E}-02$ & $4.14 \mathrm{E}-05$ & $5.60 \mathrm{E}-05$ & $3.70 \mathrm{E}-02$ \\
$650^{\circ} \mathrm{C}$ & $5.78 \mathrm{E}-05$ & $6.00 \mathrm{E}-05$ & $4.82 \mathrm{E}-02$ & $3.20 \mathrm{E}-05$ & $4.10 \mathrm{E}-05$ & $3.90 \mathrm{E}-02$ \\
$600^{\circ} \mathrm{C}$ & $3.79 \mathrm{E}-05$ & $3.00 \mathrm{E}-05$ & $6.32 \mathrm{E}-02$ & $2.02 \mathrm{E}-05$ & $2.10 \mathrm{E}-05$ & $4.81 \mathrm{E}-02$ \\
$550^{\circ} \mathrm{C}$ & $2.26 \mathrm{E}-05$ & $1.20 \mathrm{E}-05$ & $9.42 \mathrm{E}-02$ & $1.47 \mathrm{E}-05$ & $9.20 \mathrm{E}-06$ & $7.99 \mathrm{E}-02$ \\
$500{ }^{\circ} \mathrm{C}$ & $8.20 \mathrm{E}-06$ & $5.30 \mathrm{E}-06$ & $7.74 \mathrm{E}-02$ & $1.12 \mathrm{E}-05$ & $4.20 \mathrm{E}-06$ & $1.33 \mathrm{E}-01$ \\
\hline
\end{tabular}

\subsection{Oxygen Bulk Diffusion Comparison Between Tetragonal and Orthorhombic PBCO}

It is observed that from the ambient atmosphere to lower $P_{\mathrm{O}_{2}}$, the oxygen diffusion rate in the lattice slowed quickly compared to oxygen surface exchange at the gas/PBCO surface for the assynthesized tetragonal PBCO. In contrast, the oxygen surface exchange rate retarded quickly than the oxygen bulk diffusion for the pristine orthorhombic PBCO when switched to lower $P_{\mathrm{O}_{2}}$. This observation means that the former structure shifts to surface/bulk mix control when shrinking the atmosphere oxygen source and the latter is much more inclined to surface limited kinetics. When the partial pressure between the lattice and the ambient atmosphere reaches an equilibrium state, the low spin state mobile hole $\left(\mathrm{Co}^{4+}\right)$ that exists above the semi-conductor to metal transition, especially when $\delta$ is less than 0.5 , is reduced to a higher spin state $\mathrm{Co}^{3+}$ and 
leaves a vacancy site in the lattice, which will cause a decrease in electrical conductivity. The major charge carriers are usually associated with a thermally activated disproportionation reaction of $\mathrm{Co}^{3+}$ expressed in Equation 7.6.

$$
2 \mathrm{Co}_{\mathrm{Co}}^{\cdot}+\mathrm{O}_{\mathrm{O}}^{\times}=2 \mathrm{Co}_{\mathrm{Co}}^{\times}+V_{\mathrm{O}}^{*}+{ }_{2}^{1} \mathrm{O}_{2}
$$

Figure 7.3 shows the oxygen chemical diffusion coefficients and surface exchange coefficients for the as-synthesized tetragonal $\mathrm{PBCO}$ and the pristine orthorhombic $\mathrm{PBCO}$ as a function of temperature in air and $1 \%$ oxygen. Both the as-synthesized tetragonal and orthorhombic $\mathrm{PBCO}$ have excellent oxygen transport performance compared to the performance reported in the literature ${ }^{214-216}$. The as-synthesized tetragonal PBCO has a higher surface exchange and bulk diffusion activation energy $\left(E_{\mathrm{a}}\right)$ than that of the pristine orthorhombic $\mathrm{PBCO}$ in both the air and $1 \%$ oxygen. The $E_{\mathrm{a}}$ comparison indicates that the as-synthesized orthorhombic PBCO is more likely to serve as a low-temperature cathode despite the lower $\mathrm{k}, \mathrm{D}$ values than that of the assynthesized tetragonal PBCO electrode from $500{ }^{\circ} \mathrm{C}$ to $700{ }^{\circ} \mathrm{C}$. The surface exchange activation energy $\left(E_{\mathrm{a}}\right.$, surface $)$ for the as-synthesized tetragonal $\mathrm{PBCO}$ itself is higher in the air than in $1 \%$ oxygen. There is almost no $E_{\mathrm{a} \text {, surface }}$ difference between air and $1 \%$ oxygen for the pristine orthorhombic PBCO. Oxygen bulk diffusions show non-linear relationships with temperature. The oxygen bulk diffusion activation energy $\left(E_{\mathrm{a}}\right.$, bulk $)$ changes when the temperature reaches 600 ${ }^{\circ} \mathrm{C}$ for the as-synthesized tetragonal $\mathrm{PBCO}$ and $650{ }^{\circ} \mathrm{C}$ for the pristine orthorhombic $\mathrm{PBCO}$. The decrease in $E_{\text {a, bulk }}$ at higher temperatures is caused by the easier loss of oxygen. This is verified by oxygen non-stoichiometry change discussed later. $E_{\mathrm{a}}$, bulk is closely related to the lattice structure of the cathode. Cation-ordered perovskite materials are reported to have better oxygen transport performance, specifically faster oxygen bulk diffusion compared to cation-disordered perovskites ${ }^{8,66,217}$. However, oxygen transport performance and mechanism difference between 
the cation-ordered--oxygen disordered (tetragonal PBCO) and cation-ordered--oxygen ordered (orthorhombic PBCO) have not been studied yet. Here, the cation-ordered--anion-disordered assynthesized tetragonal PBCO is proven to have better oxygen transport performance (higher absolute $k, D$ values) than the ordered as-synthesized orthorhombic PBCO during their structure evolution.

The $D_{\text {chem }}$ vs T and $k_{\text {ex }}$ vs T plots were reflected in Figure 7.3. The scattering points cannot be fitted linearly. Consequently, the activity of PBCO to oxygen activation cannot be directly explained by activation energy. Rather, the enthalpies of defect formation and migration, even the entropy term should be considered here.
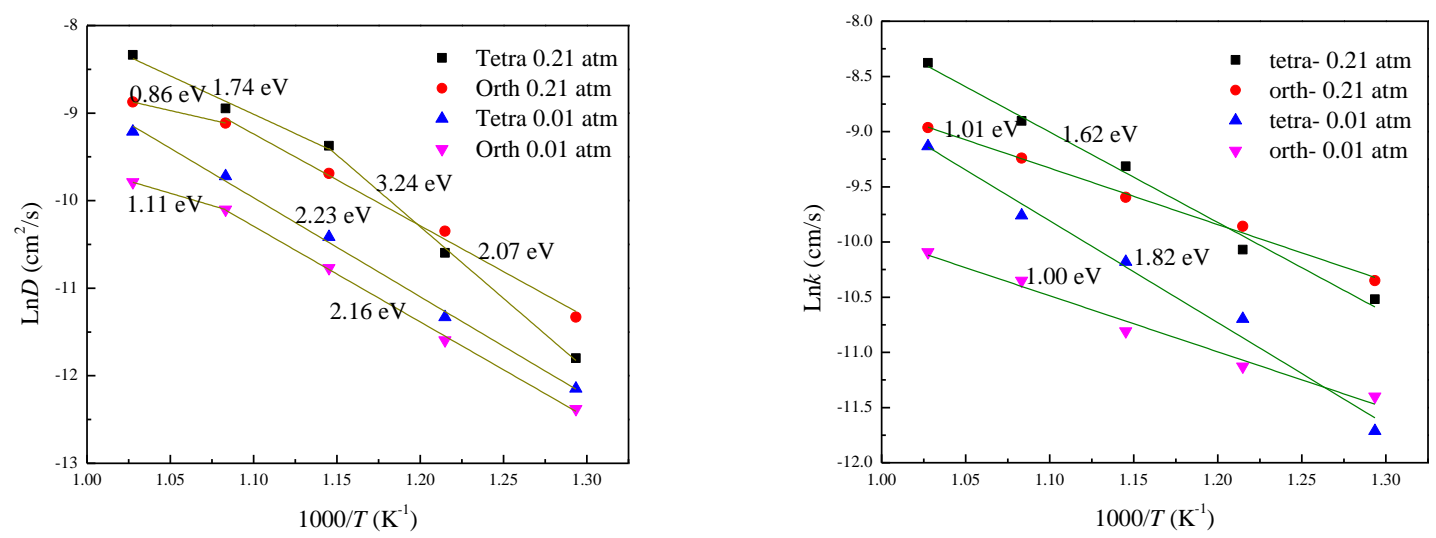

Figure 7.3 Oxygen chemical diffusion coefficient and surface exchange coefficient for the As-synthesized tetragonal $\mathrm{PBCO}$ and orthorhombic $\mathrm{PBCO}$ as a function of temperature.

It is common sense that the oxygen vacancy self-diffusion coefficient can be represented in the formula:

$$
D=\left[\mathrm{V}_{\mathrm{O}}\right]_{\mathrm{s}} D_{\mathrm{v}}
$$


$\left[\mathrm{V}_{\mathrm{O}}\right]_{\mathrm{s}}$ must be the mobile vacancy fraction and may not be the vacancy fraction directly from the oxygen non-stoichiometry determined in chapter 6 . Thus, the structure factor needs to be taken into consideration here.

Diffusivity is a rate of diffusion, it measures the rate at which particle spread. Self-diffusion $D$ is defined by the random walk in the absence of a potential gradient. For the case of a crystal lattice, it is noted as the jump distance from the site to site $(a)$, the jump frequency $(\omega)$ the number of available defects lattice sites $\left(N_{d}\right)$ and a structure-specific constant $(\alpha)$ (equal to 1 for vacancy diffusion). For oxygen diffusion, $N_{d}$ involves the formation of vacancies and is a temperaturedependent term.

$$
D=\alpha \mathrm{a}^{2} \omega N_{\mathrm{d}}
$$

The formation of defects in a system is determined by the Gibbs energy of formation, and subsequently by the entropy and enthalpy. Oxygen vacancy concentration will increase with temperature if oxygen vacancy formation enthalpy is positive. The other temperature-dependent term shown in the equation is the frequency of sufficiently energetic jumps. The energy barrier relates to the strain arising from the displacement of neighboring atoms to create space for the jump. A small fraction of the vibrations (a Boltzmann distribution) possesses enough energy to overcome this jump barrier. Consequently, the jump frequency can be described by:

$$
\omega=\vartheta \exp \left(\frac{\Delta G_{\mathrm{m}}}{R T}\right)=\vartheta \exp \left(\frac{\Delta S_{\mathrm{m}}}{R}\right) \exp \left(-\frac{\Delta H_{\mathrm{m}}}{R T}\right)
$$

$G_{\mathrm{m}}, S_{\mathrm{m}}$, and $H_{\mathrm{m}}$ are the thermodynamic parameters associated with the movement of the diffuse species. $\vartheta$ is a characteristic lattice frequency. Among these parameters, most experiment 
measurements are focused on the enthalpies of movement and defect formation, wrapped in activation energy. Such results are usually described with:

$$
D=D_{0} \exp \left(-\frac{Q}{R T}\right)
$$

From equations (7-8) and to (7-10), a more detailed view of the diffusion coefficient is obtained:

$$
D=\alpha a_{0}^{2} \vartheta \exp \left(\frac{\Delta S_{\mathrm{f}}+\Delta S_{\mathrm{m}}}{R}\right) \exp \left(-\frac{\Delta H_{\mathrm{f}}+\Delta H_{\mathrm{m}}}{R T}\right)
$$

Thus $D_{0}$ and $Q$ are given by:

$$
\begin{gathered}
D_{0}=\alpha a_{0}^{2} \vartheta \exp \left(\frac{\Delta S_{\mathrm{f}}+\Delta S_{\mathrm{m}}}{R}\right) \\
Q=\Delta H_{\mathrm{f}}+\Delta H_{\mathrm{m}}
\end{gathered}
$$

Applying the diffusion theory on specific oxides with transition metals ions, here PBCO oxides, the oxygen intrinsic diffusion can be expressed in the formula,

$$
D_{1}=\gamma a_{0}^{2} \vartheta_{0} \exp \left(\frac{\Delta S_{\mathrm{f} 2} / 2+\Delta_{S_{\mathrm{m}}}}{R}\right) \exp \left(-\frac{\Delta_{\mathrm{H}_{2} / 2}+\Delta_{H_{\mathrm{m}}}}{R T}\right)
$$

The oxygen vacancy diffusion can be expressed as,

$$
D_{2}=\left(\frac{1}{4}\right)^{\frac{1}{3}} \gamma a_{0}^{2} \vartheta_{0} P_{O_{2}}^{-\frac{1}{6}} \exp \left(\frac{\frac{\Delta_{S_{\mathrm{f} 1}}}{3}+\Delta_{S_{\mathrm{m}}}}{R}\right) \exp \left(-\frac{\frac{\Delta_{H_{\mathrm{f} 1}}}{3}+\Delta_{H_{\mathrm{m}}}}{R T}\right)
$$

For the as-synthesized tetragonal $\mathrm{PBCO}$, below $600^{\circ} \mathrm{C}$, the oxygen self-diffusion is mainly contributed by the diffusion described in formula (12), and mainly from formula (19) when above $600{ }^{\circ} \mathrm{C}$. There is an enhancement factor between the oxygen self-diffusion coefficients and the chemical diffusion coefficients. But it won't affect oxygen vacancy formation and migration 
energy. With the phase transformation set aside, the oxygen vacancy formation and migration enthalpy can be estimated here. They are calculated based on the ECR and the TG results and listed in Table 7.3. Oxygen vacancy migration enthalpy values for the as-synthesized tetragonal $\mathrm{PBCO}$ are higher than the pristine orthorhombic $\mathrm{PBCO}$, which is weird here. However, the diffusion superiority of the two different PBCO still cannot be certain or be fully understood since the phase transition has not been considered here, and the entropy term has not been discussed yet.

Table 7.3 The comparison of enthalpy for oxygen migration, formation between pristine tetragonal and orthorhombic PBCO.

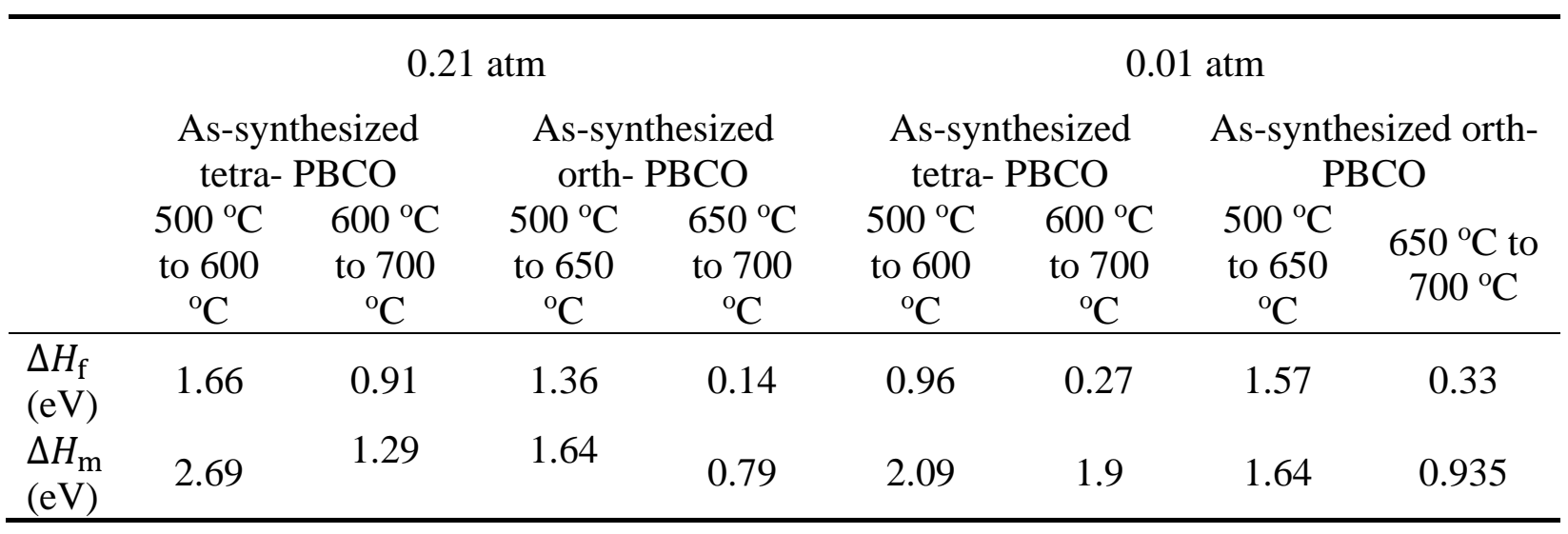

\subsection{Factors Determining Catalytic Activity in $\mathrm{O}_{2}$ Reduction on PBCO}

A surface exchange dominated process for $\mathrm{PBCO}$ electrodes has been proved from characteristic length estimated in chapter 5. However, bulk diffusion also displays an interesting phenomenon, which is reflected in the thermodynamic factor obtained in chapter 6 . Besides, both the surface exchange coefficients and oxygen bulk diffusion coefficient are nonlinearly related to temperature. It will be more complicated when applied in solid oxide fuel cells with porous cathode electrodes. So, this chapter will focus on the electro-catalytic performance of 
synthesized tetragonal and orthorhombic PBCO as SOFC cathode, and refine the surface adsorption process of oxygen on PBCO electrodes.

\subsubsection{Symmetrical Cell Performance}

After screen printing and sintering, the thickness of the porous electrode is about $30 \mu \mathrm{m}$ (Figure 7.4). Platinum can dramatically promote a cell's electrochemical performance, as reported by Watanabe et $\mathrm{al}^{30}$, and Xiong et $a l^{218}$. Specifically, the vaporization of platinum will take place above $600{ }^{\circ} \mathrm{C}$ forming $\mathrm{PtO}_{2} . \mathrm{PtO}_{2}$ will be reduced to metal again and redeposit on the electrode surface. During this vaporization-deposition process, platinum prefers to deposit around the TPBs area and contributes to an attractive performance due to its excellent catalytic ability. According to Xiong's work, this vaporization-deposition phenomenon has been confirmed by the long-term experiments in which the deposition of platinum around the TPBs is observed by elemental mappings on the cross-section of the LSF/ScSZ interface. To avoid extra performance promotion from platinum, the silver current collector was applied to all the cells in this work.
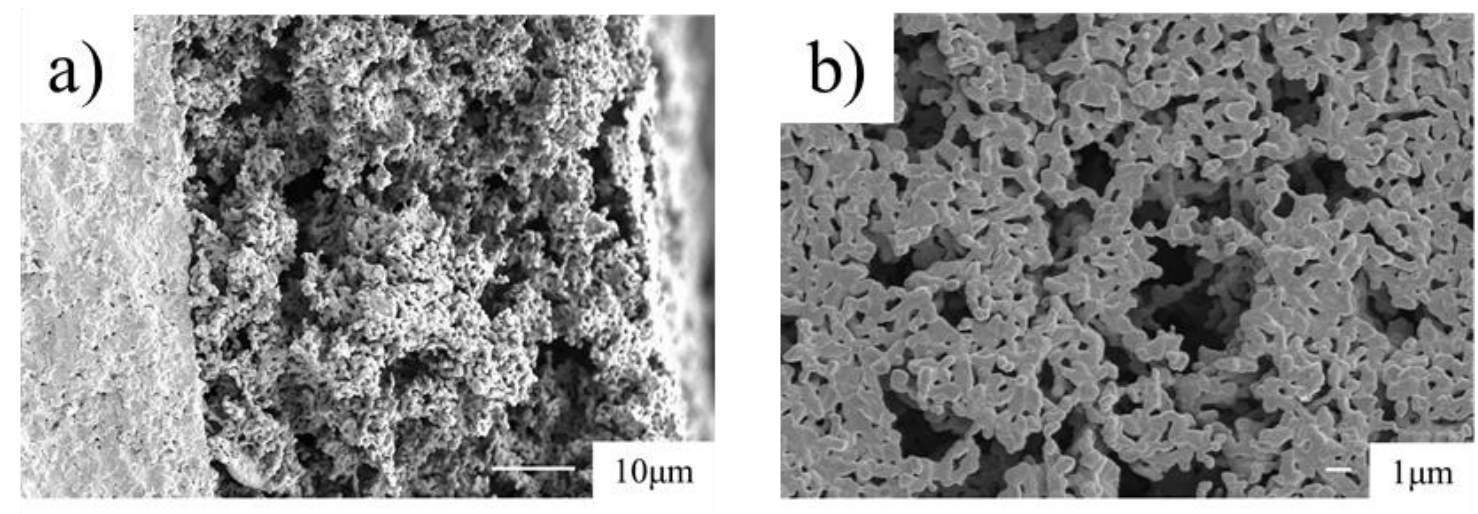

Figure 7.4 SEM morphology of a) the cross-section, b) the porous electrode of the PBCO/GDC/PBCO symmetrical cell. 
The polarization resistance $\left(\mathrm{R}_{\mathrm{p}}\right)$ of cathode materials in SOFC is an effective parameter to assess the catalytic activity on the oxygen oxidation reaction (ORR). Figure 7.5 are the Nyquist plots of $\mathrm{PBCO} / \mathrm{GDC} / \mathrm{PBCO}$ symmetric cells from 500 to $700{ }^{\circ} \mathrm{C}$ in air, $0.1 \mathrm{~atm}, 0.04 \mathrm{~atm}$, and $0.01 \mathrm{~atm}$. An attractive performance is already obtained at $650{ }^{\circ} \mathrm{C}$ with the polarization resistance of 0.078 $\Omega \cdot \mathrm{cm}^{2}$ and $0.10 \Omega \cdot \mathrm{cm}^{2}$ for the tetragonal and orthorhombic PBCO electrode, respectively. This performance is prominent compared with that of $\mathrm{LaBaCo}_{2} \mathrm{O}_{6-\delta}, \mathrm{GaBaCo}_{2} \mathrm{O}_{6-\delta}$, and other ceramic cathodes published in recent years and also competitive with that of ceramic+GDC composite cathodes, as shown in Table 7.1

There is a surface exchange and the bulk diffusion co-limited process is generally proposed in chapter 5 and chapter 6 based on the intrinsic properties of $\mathrm{PrBaCo}_{2} \mathrm{O}_{6-\delta}$. Fig 7.5 presents EIS profiles for $\mathrm{PBCO} / \mathrm{GDC} / \mathrm{PBCO}$ symmetric cells measured at different temperatures and $P_{\mathrm{O}_{2}}$, with the ohmic resistances removed. The semicircle-like profile was fitted well with the constant phase element, and the half-tear-drop-shape was fitted with the de Levie Pore element (Lo). At $550^{\circ} \mathrm{C}$, a half-tear-drop-shape spectrum occurred for the tetragonal cathode, with the $45^{\circ}$ straight line appeared at the high-frequency part. This is an indication of the bulk diffusion limitation process, followed by a very low-frequency arc representing the surface chemical reaction (oxygen dissociative adsorption and incorporation) process. Both the tetragonal and orthorhombic cathodes show very good performance: ASR is $0.078 \Omega \cdot \mathrm{cm}^{2}$ and $0.1 \Omega \cdot \mathrm{cm}^{2}$ at 650 ${ }^{\circ} \mathrm{C}$ in air respectively. Besides, EIS profiles shift to a higher frequency region with the increase of temperature and oxygen partial pressure. The highest characteristic frequency is $114.28 \mathrm{~Hz}$ at $700{ }^{\circ} \mathrm{C}$ in the air for the tetragonal cathode and $67 \mathrm{~Hz}$ for the orthorhombic cathode, which still belongs to the surface process. Another much lower frequency arc appears at high temperature and low $P_{\mathrm{O}_{2}}$. It means that at higher temperature $\mathrm{PBCO} / \mathrm{GDC}$ interface is well activated, wherein 
the charge transfer process at the interface should not be the case. Since that oxygen bulk diffusion is more sensitive to partial pressure change, the low-frequency arc that represents the chemical process is more related to oxygen diffusion through lattice oxygen vacancy. But it does not mean that the surface exchange process can be ignored. Another information is that for orthorhombic PBCO/GDC half cell, the equivalent circuit adopted for the fitting of the derived low-frequency arc changed from $R C$ to Lo when the temperature goes up from $650{ }^{\circ} \mathrm{C}$ to $700{ }^{\circ} \mathrm{C}$, which is also proof of oxygen bulk diffusion-limited process.
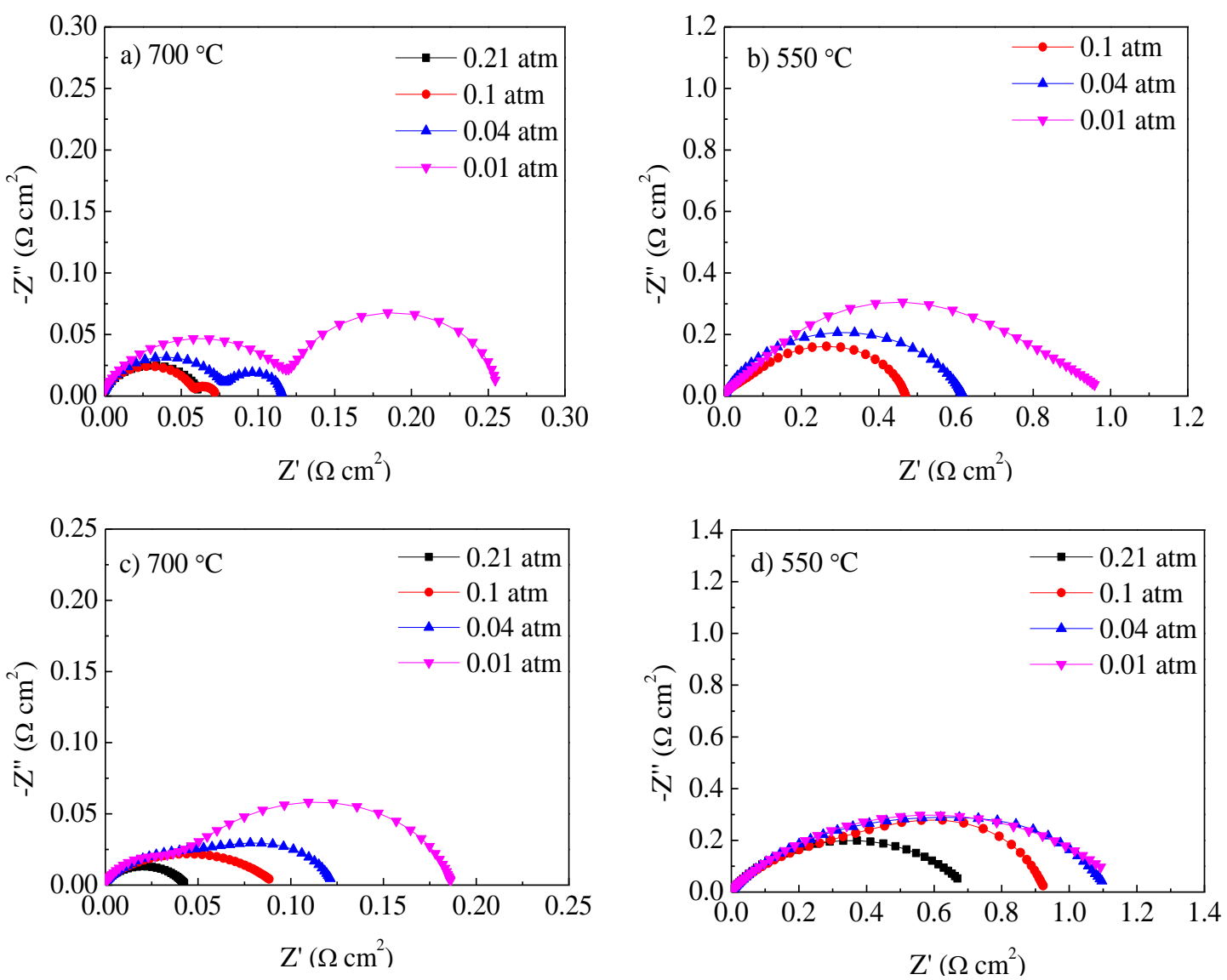

Figure 7.5 EIS profiles of $\mathrm{PrBaCo}_{2} \mathrm{O}_{6-\delta} / \mathrm{GDC}$ half cells: a) tetragonal $700{ }^{\circ} \mathrm{C}$, b) tetragonal $550{ }^{\circ} \mathrm{C}, \mathrm{c}$ ) orthorhombic $700{ }^{\circ} \mathrm{C}$, d) orthorhombic $550{ }^{\circ} \mathrm{C}$. 
In the Lo equivalent circuit, $\mathrm{L}$ is the inductance, $\mathrm{Rs}$ is the ohmic resistance from the electrolyte, Lo represents the oxygen bulk diffusion and surface chemical exchange co-limited process. The Lo element can be described as Equ. (7-16) ${ }^{219}$ :

$$
Z_{\text {chem }}=R_{\text {chem }} \sqrt{\frac{1}{1+j w t_{\text {chem }}^{\alpha}}}
$$

Although EIS signal cannot separate surface exchange behavior and bulk oxygen diffusion. It still has an activated thickness that can be compared with its critical thickness obtained from $D_{\text {chem }}, k_{\text {chem }}$. So, Adler's model was adopted here to analyze the surface chemical process and oxygen solid diffusion process observed from EIS profiles. It is assumed the impedance here under OCV is a non-charge transfer process $\mathrm{Z}_{\mathrm{chem}}$.

$$
C_{\text {chem }}=\frac{t_{\text {chem }}}{R_{\text {chem }}}
$$

Here $Z_{\text {chem }}$ is the chemical impedance, $t_{\text {chem }}$ the integrated relaxation time referred to the oxygen surface exchange and the solid-state diffusion, and $\alpha$ represents the non-ideal behavior of. $C_{c h e m}$ is related to the change of oxygen stoichiometry in MIEC. So, the oxygen reservoir ability should be much larger than double-layer capacitance that is confined to the electrolyte/electrode interface. The calculated specific chemical capacitances are listed in Table 7.4, which is around a thousand times double-layer capacitance, and showed a decreasing trend with the decrease of $P_{\mathrm{O}_{2}}$. Based on Adler's work, the large chemical capacitance also implies the activity of the electrode bulk. According to Adler's model, the $R_{\text {chem }}$ and $C_{\text {chem }}$ can be re-written as:

$$
\begin{gathered}
R_{\text {chem }}=2 \sqrt{\frac{R_{\text {surf }}}{\sigma_{i, e f f} a}} \\
C_{\text {chem }}=\frac{4 F^{2}}{R T} \frac{(1-\varepsilon) l_{\delta}}{f V_{m}}=\frac{4 F^{2}}{R T} \frac{(1-\varepsilon)}{f V_{m}} \sqrt{\frac{\sigma_{i, e f f} R_{\text {surf }}}{a}}
\end{gathered}
$$


Where $R_{\text {surf }}$ is the area-specific resistance, reflecting the oxygen exchange resistance at the PBCO surface, $f$ is the thermodynamic factor that representing how facile the stoichiometry changes with $P_{\mathrm{O}_{2}}$ obtained from the TGA result calculation, $\varepsilon$ is the porosity of the porous MIEC cathode. Here $\varepsilon$ is substituted with 0.3 and molar volume $V_{\mathrm{m}}$ with $70.28 \mathrm{~cm}^{3} / \mathrm{mol}$ calculated from the tetragonal XRD parameters. Thermodynamic factors can be referred to in chapter 5. $a$ is the surface area. $\sigma_{i, e f f}$ is the effective ionic conductivity, and can be expressed as $\sigma_{i, e f f}=(1-$ $\varepsilon) \sigma_{i} / \tau . \tau$ is the tortuosity. The characteristic length can still reach to hundred microns (169 $\mu \mathrm{m}$ at $700{ }^{\circ} \mathrm{C}$ in the air) which means the whole electrode should be activated. According to the above analysis, the following elementary steps listed in Eq. (7-20) to Eq. (7-23) all participated in the oxygen surface exchange process.

Table 7.4 Summary of values related to tetragonal PrBaCo2O6- $\delta / G D C$ EIS profiles.

\begin{tabular}{llllllll}
\hline $\begin{array}{l}\mathrm{T}=700 \\
{ }^{\circ} \mathrm{C}\end{array}$ & $\begin{array}{l}\text { Total } R \mathrm{p} \\
\left(\Omega \cdot \mathrm{cm}^{2}\right)\end{array}$ & \multicolumn{2}{c}{ Related higher frequency } & \multicolumn{3}{c}{ Chem process } & \\
& & $R \mathrm{p} 1\left(\Omega \cdot \mathrm{cm}^{2}\right)$ & $C\left(\mathrm{~F} / \mathrm{cm}^{2}\right)$ & $f(\mathrm{~Hz})$ & $R_{\text {chem }}\left(\Omega \cdot \mathrm{cm}^{2}\right)$ & $C_{\text {chem }}\left(\mathrm{F} / \mathrm{cm}^{2}\right)$ & $\alpha$ \\
\hline $0.21 \mathrm{~atm}$ & 0.06 & 0.06 & 0.013 & 114.28 & & & 0.84 \\
$0.1 \mathrm{~atm}$ & 0.07 & 0.06 & 0.015 & 87.5 & 0.013 & 12.86 & 0.86 \\
$0.04 \mathrm{~atm}$ & 0.1 & 0.08 & 0.015 & 51.30 & 0.036 & 8.57 & 0.86 \\
$0.01 \mathrm{~atm}$ & 0.26 & 0.13 & 0.018 & 30.08 & 0.13 & 6.84 & 0.81 \\
\hline
\end{tabular}

\subsection{Rate Limiting Steps on PBCO Cathodes}

\subsubsection{Oxygen Reaction Order and Rate-determining Steps Analysis}

The RDS can be roughly reflected on the reaction order determined by $P_{\mathrm{O}_{2}}$ dependent $R_{\mathrm{p}}$ through a linear relationship between $1 / R$ and $P_{\mathrm{O}_{2}}^{m}$. The order values of both the tetragonal and orthorhombic PBCO cathodes are presented in Fig 6. The values are between 1/4 and 1/2 for the 
tetragonal PBCO cathode, $1 / 8$ and $1 / 2$ for the orthorhombic cathode. In theory, each step that referred to the intermediate oxygen state corresponds to a certain reaction order value. Combined with the surface exchange and bulk diffusion co-limited process proposed in the last two parts, elementary steps participated in ORR can be listed from (7-20) to (7-23).

$$
\begin{gathered}
\text { Step } 1 \mathrm{O}_{2}(\mathrm{~g}) \leftrightarrow \mathrm{O}_{2, \text { ads }} \quad n=1 \\
\text { Step } 2 \quad \mathrm{O}_{2, \text { ads }} \leftrightarrow 2 \mathrm{O}_{\text {ads }} n=1 / 2 \\
\text { Step } 3 \mathrm{O}_{\text {ads }}+\mathrm{e} \leftrightarrow \mathrm{O}_{\text {ads }}^{-} \quad n=3 / 8 \\
\text { Step } 4 \mathrm{O}_{\text {ads }}^{-}+\mathrm{e}+V_{o}^{*} \leftrightarrow \mathrm{O}_{\mathrm{O}}^{\times} \quad n=1 / 8
\end{gathered}
$$

Oxygen reduction reaction on the as-synthesized orthorhombic PBCO cathode is much more retarded by oxygen bulk diffusion than the corresponding tetragonal structure reflected in the reaction order. Besides, deflection points occurred in Figure 7.6b when in the air for all the temperature range, it is caused by the drastically oxygen non-stoichiometry increase proved in chapter 6 . The increase of oxygen vacancy has been facilitated the oxygen incorporation step into the electrodes. 

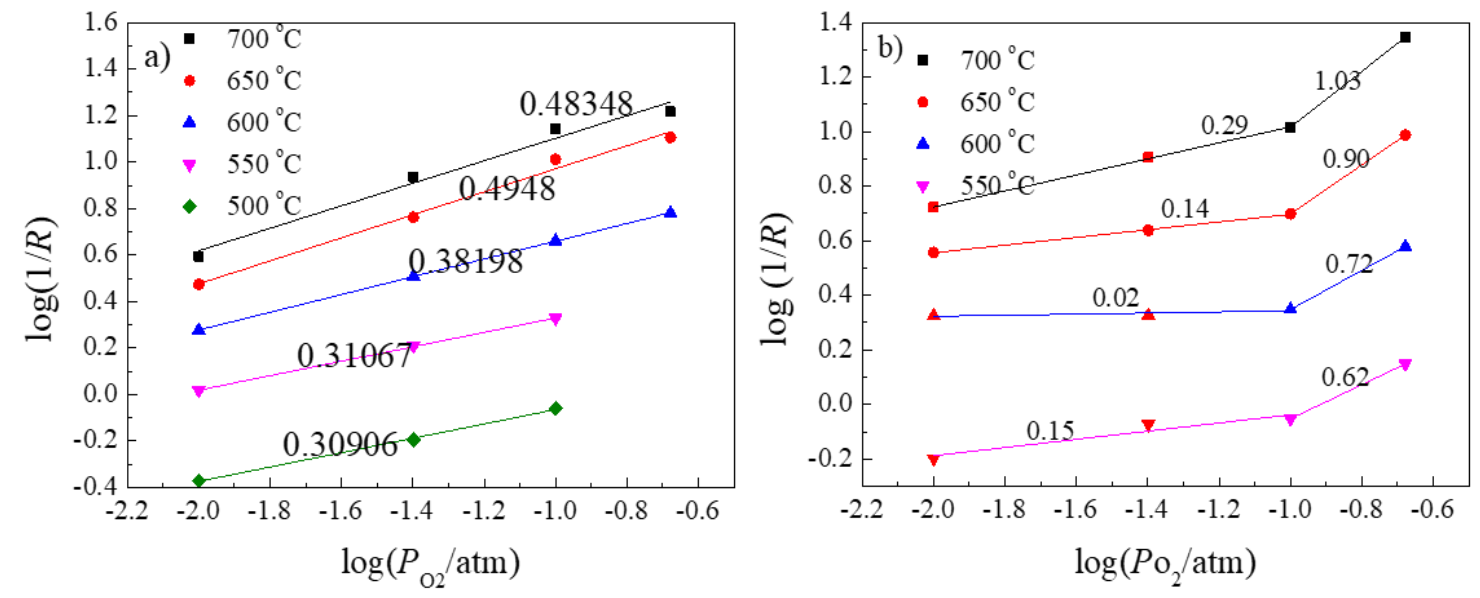

Figure 7.6 Reaction orders from the overall polarization resistance: a) tetragonal PBCO, b) orthorhombic PBCO.
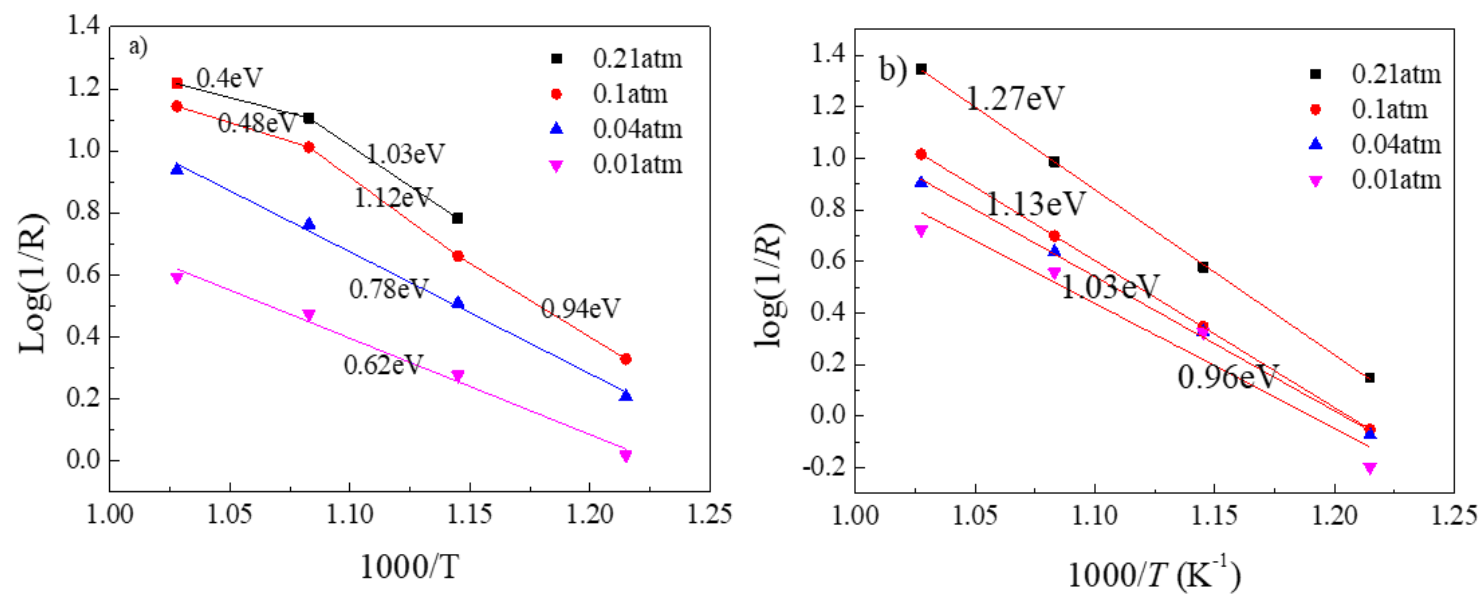

Figure 7.7 Temperature dependence of activation energy: a) tetragonal PBCO/GDC cells, b) orthorhombic PBCO/GDC cells.

\subsection{Summary}

In this chapter, oxygen transport performance and mechanism of the as-synthesized tetragonal and the pristine orthorhombic PBCO were studied with their structure evolution. The in-situ structures of PBCO were characterized using X-ray powder diffraction with hot-stage. The result shows tetragonal and orthorhombic structures at room temperature respectively in the limit of the 
XRD instrument. The tetragonal structure keeps in operation range of SOFC, while orthorhombic transformed to tetragonal when above $550{ }^{\circ} \mathrm{C}$. The comparison of the lattice parameters refinement gives a possible explanation for the oxygen transport discrepancy. The shorter bond length as-synthesized tetragonal $\mathrm{PBCO}$ has better oxygen transport performance than the assynthesized orthorhombic PBCO during the structure evolution when the temperature goes up. Both the as-synthesized tetragonal and the pristine orthorhombic PBCO electrodes show good electrocatalytic activity to oxygen reduction, with polarization resistance $0.078 \Omega \cdot \mathrm{cm}^{2}$ and 0.10 $\Omega \cdot \mathrm{cm}^{2}$ respectively at $650{ }^{\circ} \mathrm{C}$. Oxygen bulk diffusion is one of the rate-determining steps of the oxygen reduction reaction on $\mathrm{PBCO}$ electrodes. Oxygen adsorption and incorporation steps both retard the oxygen surface exchange process at the gas/electrode surface. This has been verified by the reaction order values and the intrinsic non-stoichiometry in both the as-synthesized tetragonal $\mathrm{PBCO}$ and the as-synthesized orthorhombic $\mathrm{PBCO}$ during their structure evolution with temperature and oxygen partial pressure. Besides, oxygen reduction reactions on electrodes treated in nitrogen were retard mainly by oxygen incorporation into the lattice step. 


\section{Conclusions and Recommendation Work}

In this work, a double perovskite cathode material $\mathrm{PrBaCo}_{2} \mathrm{O}_{6-\delta}(\mathrm{PBCO})$ is developed. Based on its structure evolution with temperature and oxygen partial pressure, the thermodynamic property, oxygen transport mechanism, and the oxygen reduction reaction mechanism with both tetragonal and orthorhombic PBCO were investigated and compared. In the last part, the PBCO cathode electroactive zone and the rate-determining steps are discussed.

In chapter 5, PBCO double perovskite material is chosen as the potential SOFC cathode. The crystal structure of PBCO treated in air and nitrogen is investigated with a tetragonal phase in air and an orthorhombic phase in nitrogen. Both two structures belong to layered double perovskite materials with the lattice parameters doubled in $c$ axis. The electrical conductivity test proves that the PBCO material has a metallic characteristic with a prominent minimum conductivity of $1014 \mathrm{~S} / \mathrm{cm}$. The half-tear-drop-shaped impedance spectra of PBCO symmetrical cells herald PBCO as a good MIEC cathode that possesses both excellent oxygen surface exchange and bulk diffusion performance. The good match of these two reactions expanded the reaction active zone from electrolyte/electrode $2 \mathrm{~PB}$ to the gas/electrode surface area. The bulk diffusion and surface exchange process at gas/electrode $2 \mathrm{~PB}$ was assumed to co-limit the ORR on the PBCO cathode.

In chapter 6, the structures of PBCO treated in air and nitrogen during temperature increase were primarily characterized using X-ray powder diffraction. The result shows tetragonal and orthorhombic structures at room temperature respectively in the limit of the XRD instrument. The tetragonal structure keeps in operation range of SOFC, while orthorhombic transformed to tetragonal when above $550{ }^{\circ} \mathrm{C}$. The comparison of the lattice parameters refinement gives a possible explanation for the oxygen transport discrepancy since the fast transport bond length 
was shorter for tetragonal PBCO than orthorhombic PBCO with increasing temperature. The refinement of NPD data confirms that there are two sets of crystal lattice parameters with the same $P 4 / \mathrm{mmm}$ symmetry for the as-synthesized tetragonal $\mathrm{PBCO}$ material above $600{ }^{\circ} \mathrm{C}$. Besides, the oxygen vacancies are inhomogeneously distributed between $1 \mathrm{~b}$ and $1 \mathrm{~d}$ Wyckoff positions. While the oxygen vacancies are ordered distributed between 1c and 1g Wyckoff positions with the crystal lattice belong to Pmmm symmetry. The SAED patterns and HREM morphology discovered the dislocations, strain fields, and lattice distortions in the PBCO treated in the air. Both the evidence tells that PBCO treated in the air has a higher disorder degree than that of treated in nitrogen. The study of oxygen non-stoichiometry by the TGA method proves that there will be more oxygen vacancy formed for tetragonal PBCO with temperature increase. The thermodynamic quantities obtained through solving the thermodynamic factor equation show nonlinear relationships with temperature. This is caused by a structure transformation, the appearance of a new phase, and maybe even the association of the defects.

In chapter 7, oxygen transport performance and mechanism of the as-synthesized tetragonal and as-synthesized orthorhombic PBCO were studied with their structure evolution. The activation energy calculated from the $D$ values does not keep constant for both as-synthesized tetragonal and orthorhombic PBCO oxides which correspond to the thermodynamic properties analyzed from the TG results in chapter 6. Both the as-synthesized tetragonal and the pristine orthorhombic PBCO electrodes show good electrocatalytic activity to oxygen reduction, with polarization resistance $0.078 \Omega \cdot \mathrm{cm}^{2}$ and $0.10 \Omega \cdot \mathrm{cm}^{2}$ respectively at $650{ }^{\circ} \mathrm{C}$. Oxygen bulk diffusion is one of the rate-determining steps of the oxygen reduction reaction on PBCO electrodes. Oxygen adsorption and incorporation steps both retard the oxygen surface exchange process at the gas/electrode surface. This has been verified by the reaction order values and the 
intrinsic non-stoichiometry in both the as-synthesized tetragonal PBCO and the as-synthesized orthorhombic PBCO during their structure evolution with temperature and oxygen partial pressure. Besides, oxygen reduction reactions on electrodes treated in nitrogen were retarded mainly by oxygen incorporation into the lattice step.

\subsection{Future work}

With the lattice information, the oxygen transport performance, and the oxygen reduction reaction mechanism analysis for the PBCO SOFC cathodes, the following work is recommended.

Oxygen vacancy diffusion pathways need to be clarified with the in-situ lattice information to give information on the further performance modification.

The thermal expansion coefficients of PBCO materials are higher than $20 \times 10^{-6} \mathrm{~K}^{-1}$ estimated from the lattice parameters. Such high TEC cathode could not match with the SOFC electrolyte in a long-term operation. The high TEC is mainly related to the B-site cobalt element ${ }^{220}$. The composite electrodes or B-site element doping strategy could address the TEC mismatch.

The surface exchange rate of PBCO cathodes needs to be improved with the oxygen transport performance and the oxygen reduction reaction mechanism analysis. Surface active element infiltration is one promising way to modify the material's surface activity.

The long-term stability of this cathode material in a single cell assembly operation needs to be investigated. 


\section{References}

1 Love, J., Amarasinghe, S., Selvey, D., Zheng, X. \& Christiansen, L. Development of SOFC stacks at ceramic fuel cells limited. ECS Transactions 25, 115 (2009).

2 Nagel, F.-P. Electricity from wood through the combination of gasification and solid oxide fuel cells: systems analysis and Proof-of-concept, ETH Zurich, (2008).

3 Kang, S. \& Ahn, K.-Y. Dynamic modeling of solid oxide fuel cell and engine hybrid system for distributed power generation. Applied energy 195, 1086-1099 (2017).

4 Saadabadi, S. A. et al. Solid Oxide Fuel Cells fuelled with biogas: Potential and constraints. Renewable Energy 134, 194-214 (2019).

5 Wachsman, E., Ishihara, T. \& Kilner, J. Low-temperature solid-oxide fuel cells. Mrs Bulletin 39, 773-779 (2014).

6 Brett, D. J., Atkinson, A., Brandon, N. P. \& Skinner, S. J. Intermediate temperature solid oxide fuel cells. Chemical Society Reviews 37, 1568-1578 (2008).

7 Shao, Z. \& Haile, S. M. in Materials for Sustainable Energy: A Collection of PeerReviewed Research and Review Articles from Nature Publishing Group 255-258 (World Scientific, 2011).

8 Tarancón, A., Burriel, M., Santiso, J., Skinner, S. J. \& Kilner, J. A. Advances in layered oxide cathodes for intermediate temperature solid oxide fuel cells. Journal of Materials chemistry 20, 3799-3813 (2010)

9 Yoo, S. et al. Development of double-perovskite compounds as cathode materials for low-temperature solid oxide fuel cells. Angewandte Chemie International Edition 53, 13064-13067 (2014).

10 Adler, S. B. Factors governing oxygen reduction in solid oxide fuel cell cathodes. Chemical reviews 104, 4791-4844 (2004).

11 Hermet, J., Geneste, G. \& Dezanneau, G. Molecular dynamics simulations of oxygen diffusion in GdBaCo 2 O 5.5. Applied Physics Letters 97, 174102 (2010).

12 Tomkiewicz, A. C., Meloni, M. \& McIntosh, S. On the link between bulk structure and surface activity of double perovskite based SOFC cathodes. Solid State Ionics 260, 55-59 (2014). 
13 Seymour, I., Chroneos, A., Kilner, J. \& Grimes, R. Defect processes in orthorhombic Ln BaCo 2 O 5.5 double perovskites. Physical Chemistry Chemical Physics 13, 1530515310 (2011).

14 Schichlein, H., Müller, A. C., Voigts, M., Krügel, A. \& Ivers-Tiffée, E. Deconvolution of electrochemical impedance spectra for the identification of electrode reaction mechanisms in solid oxide fuel cells. J. Appl. Electrochem. 32, 875-882 (2002).

15 Minh, N. Q. Solid oxide fuel cell technology-features and applications. Solid State Ionics 174, 271-277 (2004).

16 Handbook, F. C. EG\&G Technical Services, Inc. Under Contract No. DEAM2699FT40575, US Department of Energy, Office of Fossil Energy, National Energy Technology Laboratory, Morgantown, West Virginia, USA (2004).

17 Stambouli, A. B. \& Traversa, E. Solid oxide fuel cells (SOFCs): a review of an environmentally clean and efficient source of energy. Renewable and sustainable energy reviews 6, 433-455 (2002).

18 Pramuanjaroenkij, A., Zhou, X. Y. \& Kakaç, S. Numerical analysis of indirect internal reforming with self-sustained electrochemical promotion catalysts. International journal of hydrogen energy 35, 6482-6489 (2010).

19 Skinner, S. J. Recent advances in Perovskite-type materials for solid oxide fuel cell cathodes. International Journal of Inorganic Materials 3, 113-121 (2001).

20 Januschewsky, J., Ahrens, M., Opitz, A., Kubel, F. \& Fleig, J. Optimized La0. 6Sr0. 4CoO3- $\delta$ Thin-Film Electrodes with Extremely Fast Oxygen-Reduction Kinetics. Advanced Functional Materials 19, 3151-3156 (2009).

21 Lee, M.-J. et al. Properties of Synthesis (BaSr) \$(CoFe) O_3 \$ Cathode for IT-SOFC by GNP. 한국신재생에너지학회: 학술대회논문집, 51-54 (2006).

22 Möller, P., Kanarbik, R., Kivi, I., Nurk, G. \& Lust, E. Influence of microstructure on the electrochemical behavior of LSC cathodes for intermediate temperature SOFC. Journal of The Electrochemical Society 160, F1245 (2013).

23 Wang, W. G. \& Mogensen, M. High-performance lanthanum-ferrite-based cathode for SOFC. Solid State Ionics 176, 457-462 (2005).

24 Suzuki, T., Awano, M., Jasinski, P., Petrovsky, V. \& Anderson, H. U. Composite (La, Sr) MnO3-YSZ cathode for SOFC. Solid State Ionics 177, 2071-2074 (2006). 
25 Choi, S. et al. Highly efficient and robust cathode materials for low-temperature solid oxide fuel cells: PrBa 0.5 Sr 0.5 Co 2- x Fe x O 5+ $\delta$. Scientific reports 3, 1-6 (2013).

26 Shi, Z. et al. A Layered Perovskite EuBaCo2O5+ $\delta$ for Intermediate-Temperature Solid Oxide Fuel Cell Cathode. Fuel Cells 14, 979-990 (2014).

27 Bai, Y. et al. Electrical and electrocatalytic properties of a La0. 8Sr0. 2Co0. 17Mn0. $83 \mathrm{O} 3-\delta$ cathode for intermediate-temperature solid oxide fuel cells. Journal of Power Sources 205, 80-85 (2012).

28 Kong, X. \& Ding, X. Novel layered perovskite $\mathrm{SmBaCu} 2 \mathrm{O} 5+\delta$ as a potential cathode for intermediate temperature solid oxide fuel cells. international journal of hydrogen energy 36, 15715-15721 (2011).

29 Jiang, S. \& Badwal, S. Hydrogen oxidation at the nickel and platinum electrodes on yttria-tetragonal zirconia electrolyte. Journal of the Electrochemical Society 144, 3777 (1997).

30 Watanabe, M., Uchida, H., Shibata, M., Mochizuki, N. \& Amikura, K. High performance catalyzed-reaction layer for medium temperature operating solid oxide fuel cells. Journal of the Electrochemical Society 141, 342 (1994).

31 Saeki, M. J., Uchida, H. \& Watanabe, M. Noble metal catalysts highly-dispersed on Smdoped ceria for the application to internal reforming solid oxide fuel cells operated at medium temperature. Catalysis letters 26, 149-157 (1994).

32 Rossignol, C., Ralph, J., Bae, J.-M. \& Vaughey, J. Ln1- xSrxCoO3 (Ln= Gd, Pr) as a cathode for intermediate-temperature solid oxide fuel cells. Solid State Ionics 175, 59-61 (2004).

33 Amow, G. \& Skinner, S. Recent developments in Ruddlesden-Popper nickelate systems for solid oxide fuel cell cathodes. Journal of Solid State Electrochemistry 10, 538-546 (2006).

34 Zhao, L., Drennan, J., Kong, C. \& Amarasinghe, S. Insight into surface segregation and chromium deposition on La 0.6 $\mathrm{Sr} 0.4 \mathrm{Co} 0.2 \mathrm{Fe} 0.8 \mathrm{O} 3-\delta$ cathodes of solid oxide fuel cells. Journal of Materials Chemistry A 2, 11114-11123 (2014).

35 Stochniol, G., Syskakis, E. \& Naoumidis, A. Chemical compatibility between strontiumdoped lanthanum manganite and yttria-stabilized zirconia. Journal of the American Ceramic Society 78, 929-932 (1995). 
36 Kim, J.-H. \& Manthiram, A. $\mathrm{LnBaCo} 2 \mathrm{O} 5+\delta$ oxides as cathodes for intermediatetemperature solid oxide fuel cells. Journal of the Electrochemical Society 155, B385 (2008).

37 Dusastre, V. \& Kilner, J. Optimisation of composite cathodes for intermediate temperature SOFC applications. Solid state ionics 126, 163-174 (1999).

38 Schäfer, W., Koch, A., Herold-Schmidt, U. \& Stolten, D. Materials, interfaces and production techniques for planar solid oxide fuel cells. Solid State Ionics 86, 1235-1239 (1996).

39 Endo, A., Fukunaga, H., Wen, C. \& Yamada, K. Cathodic reaction mechanism of dense La0. 6Sr0. 4CoO3 and La0. 81Sr0. 09MnO3 electrodes for solid oxide fuel cells. Solid State Ionics 135, 353-358 (2000).

40 Adler, S. B. Mechanism and kinetics of oxygen reduction on porous La1- $\mathrm{xSrxCoO}^{-} \delta$ electrodes. Solid State Ionics 111, 125-134 (1998).

41 Irvine, J. T. \& Connor, P. Solid oxide fuels cells: facts and figures. (Springer, 2013).

42 Jiang, S. P. Development of lanthanum strontium manganite perovskite cathode materials of solid oxide fuel cells: a review. Journal of Materials Science 43, 6799-6833 (2008).

43 Voorhoeve, R., Johnson, D., Remeika, J. \& Gallagher, P. Perovskite oxides: materials science in catalysis. Science 195, 827-833 (1977).

44 Mizusaki, J. et al. Oxygen nonstoichiometry and defect equilibrium in the perovskitetype oxides La1- xSrxMnO3+ d. Solid state ionics 129, 163-177 (2000).

45 Jiang, S. P. Activation, microstructure, and polarization of solid oxide fuel cell cathodes. Journal of Solid State Electrochemistry 11, 93-102 (2007).

46 Tsai, T. \& Barnett, S. A. Effect of LSM-YSZ cathode on thin-electrolyte solid oxide fuel cell performance. Solid State Ionics 93, 207-217 (1997).

47 De Souza, R., Kilner, J. \& Walker, J. A SIMS study of oxygen tracer diffusion and surface exchange in La0. 8Sr0. 2MnO3+ $\delta$. Mater. Lett. 43, 43-52 (2000).

48 Leone, P., Santarelli, M., Asinari, P., Calì, M. \& Borchiellini, R. Experimental investigations of the microscopic features and polarization limiting factors of planar SOFCs with LSM and LSCF cathodes. Journal of Power Sources 177, 111-122 (2008). 
49 Srdić, V. V., Omorjan, R. P. \& Seydel, J. Electrochemical performances of (La, Sr) CoO3 cathode for zirconia-based solid oxide fuel cells. Materials Science and Engineering: B 116, 119-124 (2005).

50 Lv, H., Wu, Y.-j., Huang, B., Zhao, B.-y. \& Hu, K.-a. Structure and electrochemical properties of $\mathrm{Sm} 0.5 \mathrm{Sr} 0.5 \mathrm{Co} 1-\mathrm{xFexO}-\delta$ cathodes for solid oxide fuel cells. Solid State Ionics 177, 901-906 (2006).

51 Wei, B. et al. Thermal and electrical properties of new cathode material $\mathrm{Ba} 0.5 \mathrm{Sr} 0.5 \mathrm{Co}$. $8 \mathrm{Fe} 0.2 \mathrm{O} 3-\delta$ for solid oxide fuel cells. Electrochemical and Solid State Letters 8, A428 (2005).

52 Chavez, E., Mueller, M., Mogni, L. \& Caneiro, A. in Journal of Physics: Conference Series. 012043 (IOP Publishing).

53 Zhang, K., Ge, L., Ran, R., Shao, Z. \& Liu, S. Synthesis, characterization and evaluation of cation-ordered $\mathrm{LnBaCo} 2 \mathrm{O} 5+\delta$ as materials of oxygen permeation membranes and cathodes of SOFCs. Acta Materialia 56, 4876-4889 (2008).

54 Frontera, C. et al. Structural and magnetic study of PrBaCo 2 O $5+\delta(\delta \simeq 0.75)$ cobaltite. Physical Review B 70, 184428 (2004).

55 Zhang, K., Ge, L., Ran, R., Shao, Z. \& Liu, S. Synthesis, characterization and evaluation of cation-ordered $\mathrm{LnBaCo} 2 \mathrm{O} 5+\delta$ as materials of oxygen permeation membranes and cathodes of SOFCs. Acta Materialia 56, 4876-4889, doi:10.1016/j.actamat.2008.06.004 (2008).

56 Maignan, A., Martin, C., Pelloquin, D., Nguyen, N. \& Raveau, B. Structural and magnetic studies of ordered oxygen-deficient PerovskitesLnBaCo2O5 $+\delta$, closely related to the "112" structure. Journal of Solid State Chemistry 142, 247-260 (1999).

57 Taskin, A., Lavrov, A. \& Ando, Y. Transport and magnetic properties of Gd Ba Co $2 \mathrm{O}$ 5+ x single crystals: A cobalt oxide with square-lattice Co $\mathrm{O} 2$ planes over a wide range of electron and hole doping. Physical Review B 71, 134414 (2005).

58 Khalyavin, D. et al. Anisotropic magnetic, magnetoresistance, and electrotransport properties of GdBaCo 2 O 5.5 single crystals. Physical Review B 67, 214421 (2003).

59 Respaud, M. et al. Magnetic and magnetotransport properties of $\mathrm{GdBaCo} 2 \mathrm{O} 5+\delta$ : A high magnetic-field study. Physical Review B 64, 214401 (2001). 
60 Chen, D., Ran, R. \& Shao, Z. Assessment of $\mathrm{PrBaCo} 2 \mathrm{O} 5+\delta+\mathrm{Sm} 0.2 \mathrm{Ce} 0.8 \mathrm{O} 1.9$ composites prepared by physical mixing as electrodes of solid oxide fuel cells. Journal of Power Sources 195, 7187-7195, doi:10.1016/j.jpowsour.2010.05.018 (2010).

$61 \mathrm{Gu}$, H. et al. Oxygen reduction mechanism of $\mathrm{NdBaCo} 2 \mathrm{O} 5+\delta$ cathode for intermediatetemperature solid oxide fuel cells under cathodic polarization. International Journal of Hydrogen Energy 34, 2416-2420, doi:10.1016/j.ijhydene.2009.01.003 (2009).

62 Liu, J. et al. Ultrafast oxygen exchange kinetics on highly epitaxial $\mathrm{PrBaCo} 2 \mathrm{O} 5+\delta$ thin films. Applied Physics Letters 100, 193903, doi:10.1063/1.4712123 (2012).

63 Xue, J., Shen, Y. \& He, T. Double-perovskites $\mathrm{YBaCo} 2-\mathrm{xFexO}+\delta$ cathodes for intermediate-temperature solid oxide fuel cells. Journal of Power Sources 196, 37293735, doi:10.1016/j.jpowsour.2010.12.071 (2011).

64 Zhou, Q., He, T. \& Ji, Y. SmBaCo2O5+x double-perovskite structure cathode material for intermediate-temperature solid-oxide fuel cells. Journal of Power Sources 185, 754 758, doi:10.1016/j.jpowsour.2008.07.064 (2008).

65 Tsvetkov, D., Sereda, V. \& Zuev, A. Y. Oxygen nonstoichiometry and defect structure of the double perovskite GdBaCo2O6- $\delta$. Solid State Ionics 180, 1620-1625 (2010).

66 Parfitt, D., Chroneos, A., Tarancón, A. \& Kilner, J. A. Oxygen ion diffusion in cation ordered/disordered GdBaCo 2 O 5+ $\delta$. Journal of Materials Chemistry 21, 2183-2186 (2011).

67 Pang, S., Jiang, X., Li, X., Wang, Q. \& Zhang, Q. Structural stability and hightemperature electrical properties of cation-ordered/disordered perovskite $\mathrm{LaBaCoO}$. Materials Chemistry and Physics 131, 642-646 (2012).

68 Bernuy-Lopez, C. et al. Effect of cation ordering on the performance and chemical stability of layered double perovskite cathodes. Materials 11, 196 (2018).

69 Fauth, F. et al. Interplay of structural, magnetic and transport properties in thelayered Cobased perovskite LnBaCo 2 O 5 ( $\mathrm{Ln}=\mathrm{Tb}$, Dy, Ho). The European Physical Journal BCondensed Matter and Complex Systems 21, 163-174 (2001).

70 Frontera, C., García-Muñoz, J., Llobet, A. \& Aranda, M. A. G. Selective spin-state switch and metal-insulator transition in GdBaCo 2 O 5.5. Physical Review B 65, 180405 (2002). 
71 Zhou, Z. \& Schlottmann, P. Orbital order and uniaxial spin anisotropy in EuBaCo 2 O 5.5 single crystals. Physical Review B 71, 174401 (2005).

72 Tarancon, A., Marrerolopez, D., Penamartinez, J., Ruizmorales, J. \& Nunez, P. Effect of phase transition on high-temperature electrical properties of $\mathrm{GdBaCo} 2 \mathrm{O} 5+\mathrm{x}$ layered perovskite. Solid State Ionics 179, 611-618, doi:10.1016/j.ssi.2008.04.028 (2008).

73 Frontera, C. et al. Structural and magnetic study ofPrBaCo2O5+ $\delta(\delta \simeq 0.75)$ cobaltite. Physical Review B 70, doi:10.1103/PhysRevB.70.184428 (2004).

74 Guo, W. et al. Thermal and electrochemical properties of layered perovskite $\operatorname{PrBaCo} 2-\mathrm{xMnxO}+\delta(\mathrm{x}=0.1,0.2$ and 0.3$)$ cathode materials for intermediate temperature solid oxide fuel cells. International Journal of Hydrogen Energy 40, 1245712465, doi:10.1016/j.ijhydene.2015.07.136 (2015).

75 Rivas-Murias, B., Sánchez-Andújar, M., Rivas, J. \& Señarís-Rodríguez, M. A. Influence of the oxygen content and the preparation method on the power factor of $\mathrm{PrBaCo} 2 \mathrm{O} 5+\delta$ samples (0.54 $\leq \delta \leq 0.84)$. Journal of Alloys and Compounds 509, 5250-5255, doi:10.1016/j.jallcom.2011.01.217 (2011).

76 Tan, P., Liu, M., Shao, Z. \& Ni, M. Recent advances in perovskite oxides as electrode materials for nonaqueous lithium-oxygen batteries. Advanced Energy Materials 7, 1602674 (2017).

77 Cao, Y. et al. Recent advances of perovskite oxides as electrode materials for supercapacitor. Chemical Communications (2021).

78 Ishihara, T. Perovskite oxide for solid oxide fuel cells. (Springer Science \& Business Media, 2009).

79 Haverkort, M. W. Spin and orbital degrees of freedom in transition metal oxides and oxide thin films studied by soft x-ray absorption spectroscopy. arXiv preprint condmat/0505214 (2005).

80 Hervieu, M. et al. Metallicity and thermopower of the misfit cobaltite [Bi 2 Ba $1.8 \mathrm{Co} 0.2$ O 4] RS [CoO 2] 2. Physical Review B 67, 045112 (2003).

81 Maignan, A., Wang, L., Hébert, S., Pelloquin, D. \& Raveau, B. Large thermopower in metallic misfit cobaltites. Chemistry of materials 14, 1231-1235 (2002).

82 Tsubouchi, S. et al. Simultaneous metal-insulator and spin-state transitions in $\mathrm{Pr} 0.5 \mathrm{Ca}$ 0.5 CoO 3. Physical Review B 66, 052418 (2002). 
83 Suard, E., Fauth, F., Caignaert, V., Mirebeau, I. \& Baldinozzi, G. Charge ordering in the layered Co-based perovskite HoBaCo 2 O 5. Physical Review B 61, R11871 (2000).

84 Vogt, T. et al. Low to high spin-state transition induced by charge ordering in antiferromagnetic YBaCo 2 O 5. Phys. Rev. Lett. 84, 2969 (2000).

85 Troyanchuk, I. et al. Phase Transitions in the Gd 0.5 Ba 0.5 CoO 3 Perovskite. Phys. Rev. Lett. 80, 3380 (1998).

86 Moritomo, Y. et al. Metal-insulator transition induced by a spin-state transition in TbBaCo 2O 5 + d e 1 t a (delta= 0.5). PHYSICAL REVIEW-SERIES B- 61, R13 325-R313 328 (2000).

87 Kundu, A. K., Raveau, B., Caignaert, V., Rautama, E. \& Pralong, V. Electron transport and thermoelectric properties of layered perovskite LaBaCo2O5. 5. Journal of Physics: Condensed Matter 21, 056007 (2009).

88 Rautama, E.-L. et al. Cationic ordering and microstructural effects in the ferromagnetic perovskite La0. 5Ba0. 5CoO3: impact upon magnetotransport properties. Chemistry of materials 20, 2742-2750 (2008).

89 Aksenova, T., Gavrilova, L. Y., Tsvetkov, D., Voronin, V. \& Cherepanov, V. Crystal structure and physicochemical properties of layered perovskite-like phases LnBaCo $2 \mathrm{O}$ 5+ $\delta$. Russian Journal of Physical Chemistry A 85, 427-432 (2011).

90 Pouchard, M., Villesuzanne, A. \& Doumerc, J.-P. Spin state behavior in some cobaltites (III) and (IV) with perovskite or related structure. Journal of Solid State Chemistry 162, 282-292 (2001).

91 Harrell, Z. et al. Oxygen content tailored magnetic and electronic properties in cobaltite double perovskite thin films. Applied Physics Letters 110, 093102 (2017).

92 Suard, E., Fauth, F. \& Caignaert, V. Rhombohedral distortion in the new disordered LaBaCo2O6 perovskite. Physica B: Condensed Matter 276, 254-255 (2000).

93 Taskin, A., Lavrov, A. \& Ando, Y. Ising-Like Spin Anisotropy and Competing Antiferromagnetic-Ferromagnetic Orders in G d B a C o 2 O 5.5 Single Crystals. Phys. Rev. Lett. 90, 227201 (2003).

94 Seikh, M. M., Raveau, B., Caignaert, V. \& Pralong, V. Switching from unusual to usual ferromagnetism in “112” LnBaCo2O5. 50 $\delta$ : By calcium doping. Journal of magnetism and magnetic materials 320, 2676-2681 (2008). 
95 Boehm, E. et al. Oxygen diffusion and transport properties in non-stoichiometric Ln2$\mathrm{xNiO} 4+\delta$ oxides. Solid State Ionics 176, 2717-2725 (2005).

96 Sayers, R., De Souza, R., Kilner, J. \& Skinner, S. Low temperature diffusion and oxygen stoichiometry in lanthanum nickelate. Solid State Ionics 181, 386-391 (2010).

97 Munnings, C., Skinner, S., Amow, G., Whitfield, P. \& Davidson, I. Oxygen transport in the La2Ni1- xCoxO4+ $\delta$ system. Solid State Ionics 176, 1895-1901 (2005).

98 Matsuura, T., Tabuchi, J., Mizusaki, J., Yamauchi, S. \& Fueki, K. Electrical properties of La2- x, Srx, CoO4-I: Structure, electrical conductivity, and Seebeck coefficient of single crystals $\mathrm{x}=0.0,0.5,1.0$ and 1.5. Journal of Physics and Chemistry of Solids 49, 1403-1408 (1988).

99 Tarancón, A., Skinner, S. J., Chater, R. J., Hernandez-Ramirez, F. \& Kilner, J. A. Layered perovskites as promising cathodes for intermediate temperature solid oxide fuel cells. Journal of Materials Chemistry 17, 3175-3181 (2007).

100 Tarancón, A., Marrero-López, D., Peña-Martínez, J., Ruiz-Morales, J. \& Núñez, P. Effect of phase transition on high-temperature electrical properties of $\mathrm{GdBaCo} 2 \mathrm{O} 5+\mathrm{x}$ layered perovskite. Solid State Ionics 179, 611-618 (2008).

101 Kim, G. et al. Oxygen exchange kinetics of epitaxial $\operatorname{Pr} \mathrm{Ba} \mathrm{Co} 2 \mathrm{O} 5+\delta$ thin films. Applied physics letters 88, 024103 (2006).

102 Yoo, S. et al. Development of double-perovskite compounds as cathode materials for low-temperature solid oxide fuel cells. Angewandte Chemie 126, 13280-13283 (2014).

103 Amin, R. \& Karan, K. Characterization of La0. 5Ba0. 5CoO3- $\delta$ as a SOFC cathode material. Journal of the Electrochemical Society 157, B285 (2009).

104 Shaikh, M., Fathima, A., Swamynadhan, M., Das, H. \& Ghosh, S. Investigation into Cation-Ordered Magnetic Polar Double Perovskite Oxides. Chemistry of Materials (2021).

105 Taskin, A., Lavrov, A. \& Ando, Y. Achieving fast oxygen diffusion in perovskites by cation ordering. Applied Physics Letters 86, 091910 (2005).

106 Taskin, A., Lavrov, A. \& Ando, Y. Fast oxygen diffusion in A-site ordered perovskites. Progress in Solid State Chemistry 35, 481-490 (2007). 
107 ten Elshof, J. E., Lankhorst, M. \& Bouwmeester, H. J. Oxygen exchange and diffusion coefficients of strontium-doped lanthanum ferrites by electrical conductivity relaxation. Journal of the Electrochemical Society 144, 1060 (1997).

108 Bouwmeester, H. J. \& Burggraaf, A. in Membrane Science and Technology Vol. 4 435528 (Elsevier, 1996).

109 Mauvy, F. et al. Chemical oxygen diffusion coefficient measurement by conductivity relaxation-correlation between tracer diffusion coefficient and chemical diffusion coefficient. J. Eur. Ceram. Soc. 24, 1265-1269 (2004).

110 Ahlgren, E. O. \& Poulsen, F. W. Thermoelectric power and electrical conductivity of strontium-doped lanthanum manganite. Solid State Ionics 86, 1173-1178 (1996).

111 Petric, A., Huang, P. \& Tietz, F. Evaluation of $\mathrm{La}-\mathrm{Sr}-\mathrm{Co}-\mathrm{Fe}-\mathrm{O}$ perovskites for solid oxide fuel cells and gas separation membranes. Solid State Ionics 135, 719-725 (2000).

112 Lane, J., Benson, S., Waller, D. \& Kilner, J. Oxygen transport in La0. 6Sr0. 4Co0. 2 Fe0. 8O3- $\delta$. Solid State Ionics 121, 201-208 (1999).

113 Wang, L., Merkle, R., Maier, J., Acartürk, T. \& Starke, U. Oxygen tracer diffusion in dense Ba 0.5 Sr 0.5 Co 0.8 Fe 0.2 O 3- $\delta$ films. Applied Physics Letters 94, 071908 (2009).

114 Peña-Martínez, J., Tarancón, A., Marrero-López, D., Ruiz-Morales, J. \& Núñez. Evaluation of $\mathrm{GdBaCo} 2 \mathrm{O} 5+\delta$ as Cathode Material for Doped Lanthanum Gallate Electrolyte IT-SOFCs. Fuel Cells 8, 351-359 (2008).

115 Tarancón, A., Burriel, M., Santiso, J., Skinner, S. J. \& Kilner, J. A. Advances in layered oxide cathodes for intermediate temperature solid oxide fuel cells. Journal of Materials Chemistry 20, 3799, doi:10.1039/b922430k (2010).

116 Seymour, I. D., Chroneos, A., Kilner, J. A. \& Grimes, R. W. Defect processes in orthorhombic LnBaCo2O5.5 double perovskites. Phys Chem Chem Phys 13, 1530515310, doi:10.1039/c1cp21471c (2011).

117 Norby, T. Fast oxygen ion conductors - from doped to ordered systemsBasis of a presentation given at Materials Discussion No. 3, 26-29 September, 2000, University of Cambridge, UK. Journal of Materials Chemistry 11, 11-18 (2001). 
118 Brichzin, V., Fleig, J., Habermeier, H. U. \& Maier, J. Geometry dependence of cathode polarization in solid oxide fuel cells investigated by defined Sr-doped $\mathrm{LaMnO} 3$ microelectrodes. Electrochemical and Solid State Letters 3, 403 (2000).

119 Yashiro, K. et al. Composite Cathode of Perovskite-Related Oxides,(La, Sr) CoO3$\delta(\mathrm{La}, \mathrm{Sr}) 2 \mathrm{CoO} 4-\delta$, for Solid Oxide Fuel Cells. Electrochemical and Solid State Letters 12, B135 (2009).

120 Liu, Z., Liu, M., Yang, L. \& Liu, M. LSM-infiltrated LSCF cathodes for solid oxide fuel cells. Journal of Energy Chemistry 22, 555-559 (2013).

121 Tanner, C. W., Fung, K. Z. \& Virkar, A. V. The effect of porous composite electrode structure on solid oxide fuel cell performance: I. theoretical analysis. Journal of the Electrochemical Society 144, 21 (1997).

122 Li, Y., Gemmen, R. \& Liu, X. Oxygen reduction and transportation mechanisms in solid oxide fuel cell cathodes. Journal of Power Sources 195, 3345-3358 (2010).

123 Perry, M. L., Newman, J. \& Cairns, E. J. Mass transport in gas-diffusion electrodes: a diagnostic tool for fuel-cell cathodes. J. Electrochem. Soc. 145, 5-15 (1998).

124 Teraoka, Y., Zhang, H., Okamoto, K. \& Yamazoe, N. Mixed ionic-electronic conductivity of La1- xSrxCo1- yFeyO3- $\delta$ perovskite-type oxides. Materials research bulletin 23, 51-58 (1988).

$125 \mathrm{He}, \mathrm{H}$. et al. Low-temperature fabrication of oxide composites for solid-oxide fuel cells. Journal of the American Ceramic Society 87, 331-336 (2004).

126 Chen, Y., Urquidi-Macdonald, M. \& Macdonald, D. D. The electrochemistry of zirconium in aqueous solutions at elevated temperatures and pressures. Journal of Nuclear Materials 348, 133-147 (2006).

127 Gong, M., Gemmen, R. S. \& Liu, X. Modeling of oxygen reduction mechanism for 3PB and $2 \mathrm{~PB}$ pathways at solid oxide fuel cell cathode from multi-step charge transfer. Journal of Power Sources 201, 204-218 (2012).

128 Kleitz, M., Kloidt, T. \& Dessemond, L. High Temperature Electrochemical Behaviour of Fast Ion and Mixed Conductors. 14th RISO International Sym-posium on Materials Science, 89 (1993). 
129 Jaouen, F. \& Lindbergh, G. Transient Techniques for Investigating Mass-Transport Limitations in Gas Diffusion Electrodes: I. Modeling the Cathode. Journal of The Electrochemical Society 150, A1699 (2003).

130 Bauerle, J. Study of solid electrolyte polarization by a complex admittance method. $J$. Phys. Chem. Solids 30, 2657-2670 (1969).

131 Macdonald, J. Impedance Spectroscopy: Emphasizing solid materials and systems. 1987. New York, John Wiley \& Sons.

132 Schouler, E., Giroud, G. \& Kleitz, M. Applications selon Bauerle du tracé des diagrammes d'admittance complexe en électrochimie des solides-II.-Étude de la conductivité de la zircone stabilisée à l'yttrium. J. Chim. Phys. 70, 1309-1316 (1973).

133 Jiang, S., Love, J. \& Ramprakash, Y. Electrode behaviour at (La, Sr) MnO3/Y2O3-ZrO2 interface by electrochemical impedance spectroscopy. Journal of Power Sources 110, 201-208 (2002).

134 Van Herle, J. \& McEvoy, A. Oxygen diffusion through silver cathodes for solid oxide fuel cells. Journal of Physics and Chemistry of Solids 55, 339-347 (1994).

135 Jimenez, R., Kloidt, T. \& Kleitz, M. Reaction-zone expansions and mechanism of the $\mathrm{O}$ 2, Ag/yttria-stabilized zirconia electrode reaction. Journal of the Electrochemical Society 144, 582 (1997).

136 Wilson, J. R. et al. Quantitative three-dimensional microstructure of a solid oxide fuel cell cathode. Electrochemistry Communications 11, 1052-1056 (2009).

137 Song, T. W. et al. Performance analysis of a tubular solid oxide fuel cell/micro gas turbine hybrid power system based on a quasi-two dimensional model. Journal of Power Sources 142, 30-42 (2005).

138 Kong, W., Zhang, M., Han, Z. \& Zhang, Q. A theoretical model for the triple phase boundary of solid oxide fuel cell electrospun electrodes. Applied Sciences 9, 493 (2019).

139 Fleig, J. Solid oxide fuel cell cathodes: Polarization mechanisms and modeling of the electrochemical performance. Annual Review of Materials Research 33, 361-382 (2003).

140 Hayd, J., Dieterle, L., Guntow, U., Gerthsen, D. \& Ivers-Tiffée, E. Nanoscaled La0. 6Sr0. $4 \mathrm{CoO} 3-\delta$ as intermediate temperature solid oxide fuel cell cathode: Microstructure and electrochemical performance. Journal of Power Sources 196, 7263-7270 (2011). 
141 Chan, S., Chen, X. \& Khor, K. Cathode micromodel of solid oxide fuel cell. Journal of the Electrochemical Society 151, A164 (2003).

142 Hernández-Pacheco, E., Singh, D., Hutton, P. N., Patel, N. \& Mann, M. D. A macro-level model for determining the performance characteristics of solid oxide fuel cells. Journal of Power Sources 138, 174-186 (2004).

143 Adler, S. B., Lane, J. \& Steele, B. Electrode kinetics of porous mixed-conducting oxygen electrodes. Journal of the electrochemical society 143, 3554 (1996).

144 Liu, M. \& Winnick, J. Comment on "Electrode Kinetics of Porous Mixed-Conducting Oxygen Electrodes"[SB Adler, JA Lane, and BCH Steel (pp. 3554-3564, Vol. 143, No. 11, 1996)]. Journal of the Electrochemical Society 144, 1881 (1997).

145 Adler, S. Limitations of charge-transfer models for mixed-conducting oxygen electrodes. Solid State Ionics 135, 603-612 (2000).

146 Svensson, A. M., Sunde, S. \& Nisancioglu, K. A mathematical model of the porous SOFC cathode. Solid State Ionics 86, 1211-1216 (1996).

147 Svensson, A. M., Sunde, S. \& Nisşancioğlu, K. Mathematical Modeling of Oxygen Exchange and Transport in Air-Perovskite-YSZ Interface Regions: I. Reduction of Intermediately Adsorbed Oxygen. Journal of the Electrochemical Society 144, 2719 (1997).

148 Svensson, A. M., Sunde, S. \& Nis, K. Mathematical Modeling of Oxygen Exchange and Transport in Air-Perovskite-Yttria-Stabilized Zirconia Interface Regions: II. Direct Exchange of Oxygen Vacancies. Journal of the Electrochemical Society 145, 1390 (1998).

149 Coffey, G. W., Pederson, L. R. \& Rieke, P. C. Competition between bulk and surface pathways in mixed ionic electronic conducting oxygen electrodes. Journal of the Electrochemical Society 150, A1139 (2003).

150 Kopasakis, G., Brinson, T., Credle, S. \& Xu, M. in Turbo Expo: Power for Land, Sea, and Air. 565-575.

151 Liu, M. \& Winnick, J. Fundamental issues in modeling of mixed ionic-electronic conductors (MIECs). Solid State Ionics 118, 11-21 (1999). 
152 Van Heuveln, F. \& Bouwmeester, H. J. Electrode Properties of Sr-Doped LaMnO3 on Yttria-Stabilized Zirconia: II. Electrode Kinetics. Journal of the Electrochemical Society 144, 134 (1997).

153 Mitterdorfer, A. Identification of the oxygen reduction at cathodes of solid oxide fuel cells, ETH Zurich, (1997).

154 Chan, S. \& Xia, Z. Anode micro model of solid oxide fuel cell. Journal of the Electrochemical Society 148, A388 (2001).

155 Yildiz, B., la O, G. \& Shao-Horn, Y. in ABSTRACTS OF PAPERS OF THE AMERICAN CHEMICAL SOCIETY. U673-U673 (AMER CHEMICAL SOC 1155 16TH ST, NW, WASHINGTON, DC 20036 USA).

156 Wang, J.-H., Liu, M. \& Lin, M.-C. Oxygen reduction reactions in the SOFC cathode of $\mathrm{Ag} / \mathrm{CeO}$. Solid State Ionics 177, $939-947$ (2006).

157 Fleig, J. On the current-voltage characteristics of charge transfer reactions at mixed conducting electrodes on solid electrolytes. Physical Chemistry Chemical Physics 7, 2027-2037 (2005).

158 Baumann, F., Maier, J. \& Fleig, J. The polarization resistance of mixed conducting SOFC cathodes: A comparative study using thin film model electrodes. Solid State Ionics 179, 1198-1204 (2008).

159 Baumann, F. S., Fleig, J., Habermeier, H.-U. \& Maier, J. Impedance spectroscopic study on well-defined ( $\mathrm{La}, \mathrm{Sr})(\mathrm{Co}, \mathrm{Fe}) \mathrm{O} 3-\delta$ model electrodes. Solid State Ionics 177, 10711081 (2006).

160 Mebane, D. S., Liu, Y. \& Liu, M. A two-dimensional model and numerical treatment for mixed conducting thin films: The effect of sheet resistance. Journal of The Electrochemical Society 154, A421 (2007).

161 Mebane, D. S. \& Liu, M. Classical, phenomenological analysis of the kinetics of reactions at the gas-exposed surface of mixed ionic electronic conductors. Journal of Solid State Electrochemistry 10, 575-580 (2006).

162 Zhang, N. et al. A New Insight into the Oxygen Reduction Reaction on High Performance Cation-Ordered PBCO Perovskite as IT-SOFC Cathode. ECS Transactions 78, 643 (2017).

163 Young, R. The rietveld method. Vol. 5 (1993). 
164 Leonidov, I., Patrakeev, M., Mitberg, E. B., Leonidova, O. \& Kozhevnikov, V. Thermodynamic and structural properties of PrBaCo $2 \mathrm{O} 5+\delta$. Inorganic materials $\mathbf{4 2}$, 196-201 (2006).

165 Zhang, X. et al. Effect of oxygen content on transport and magnetic properties of PrBaCo2O5. 50+ $\delta$. Materials Research Bulletin 65, 80-88 (2015).

166 Kalinkin, M. et al. Equilibrium of Defects and Electrical Conductivity of CationDeficient Double Cobaltites. Russian Journal of Physical Chemistry A 93, 1394-1398 (2019).

167 Jacobson, A. J., Morgan, D. \& Grey, C. Enhanced Mixed Electronic-Ionic Conductors through Cation Ordering. (Univ. of Houston, TX (United States), 2014).

168 McCusker, L., Von Dreele, R., Cox, D., Louër, D. \& Scardi, P. Rietveld refinement guidelines. Journal of Applied Crystallography 32, 36-50 (1999).

169 Hill, R. \& Flack, H. The use of the Durbin-Watson d statistic in Rietveld analysis. Journal of Applied Crystallography 20, 356-361 (1987).

170 Zeng, P. et al. Re-evaluation of $\mathrm{Ba} 0.5 \mathrm{Sr} 0.5 \mathrm{Co} 0.8 \mathrm{Fe} 0.2 \mathrm{O} 3-\delta$ perovskite as oxygen semipermeable membrane. Journal of Membrane Science 291, 148-156, doi:10.1016/j.memsci.2007.01.003 (2007).

171 Wang, Y., Wang, Y. \& Xia, C. Surface process of doped ceria reduction by electrical conductivity relaxation. Journal of The Electrochemical Society 159, F570 (2012).

172 Den Otter, M., Bouwmeester, H. J., Boukamp, B. A. \& Verweij, H. Reactor flush time correction in relaxation experiments. Journal of the Electrochemical Society 148, J1 (2001).

173 Moritomo, Y. et al. Metal-insulator transition induced by a spin-state transition in TbBaCo 2 O 5+ $\delta(\delta=0.5)$. Physical Review B 61, R13325 (2000).

174 Kusuya, H. et al. Structural change at metal-insulator transition of Tb 2 Ba 2 Co 4 O 11. Journal of the Physical Society of Japan 70, 3577-3580 (2001).

175 Bernuy-Lopez, C., Høydalsvik, K., Einarsrud, M.-A. \& Grande, T. Effect of A-site cation ordering on chemical stability, oxygen stoichiometry and electrical conductivity in layered LaBaCo2O5+ $\delta$ double perovskite. Materials 9, 154 (2016).

176 Joung, Y.-H., Kang, H. I., Choi, W. S. \& Kim, J. H. Investigation of X-ray photoelectron spectroscopy and electrical conductivity properties of the layered perovskite $\mathrm{LnBaCo} 2 \mathrm{O}$ 
5+ d ( $\mathrm{Ln}=\mathrm{Pr}, \mathrm{Nd}, \mathrm{Sm}$, and Gd) for IT-SOFC. Electronic Materials Letters 9, 463-465 (2013).

177 Kim, J. H. et al. Promotion of oxygen reduction reaction on a double perovskite electrode by a water-induced surface modification. Energy \& Environmental Science 14, 1506$1516(2021)$.

178 Atanassova, Y. et al. Raman-and infrared-active phonons in $\mathrm{YBaCuFeO}$ 5: Experiment and lattice dynamics. Physical Review B 47, 15201 (1993).

179 Kawada, T. et al. Determination of oxygen vacancy concentration in a thin film of La0. $6 \mathrm{Sr} 0.4 \mathrm{CoO} 3-\delta$ by an electrochemical method. Journal of the Electrochemical Society 149, E252 (2002).

180 Jin, F., Xu, H., Long, W., Shen, Y. \& He, T. Characterization and evaluation of double perovskites $\mathrm{LnBaCoFeO5}+\delta(\mathrm{Ln}=\mathrm{Pr}$ and $\mathrm{Nd})$ as intermediate-temperature solid oxide fuel cell cathodes. Journal of Power Sources 243, 10-18 (2013).

181 Chen, D., Ran, R. \& Shao, Z. Assessment of PrBaCo2O5+ $\delta+$ Sm0. 2Ce0. 8O1. 9 composites prepared by physical mixing as electrodes of solid oxide fuel cells. Journal of Power Sources 195, 7187-7195 (2010).

182 Wan, Y., Hu, B. \& Xia, C. Oxygen reduction at the three-phase boundary of PrBaCo2O5+ $\delta$-Sm0. 2Ce0. 8 O1. 9 composite. Electrochimica Acta 252, 171-179 (2017).

183 Boukamp, B. A., Verbraeken, M., Blank, D. H. \& Holtappels, P. SOFC-anodes, proof for a finite-length type Gerischer impedance? Solid State Ionics 177, 2539-2541 (2006).

184 Boukamp, B. A. \& Bouwmeester, H. J. Interpretation of the Gerischer impedance in solid state ionics. Solid state ionics 157, 29-33 (2003).

185 Yashiro, K. et al. Mass transport properties of Ce0. 9Gd0. 1O2- $\delta$ at the surface and in the bulk. Solid State Ionics 152, 469-476 (2002).

186 Kim, G. T., Wang, S., Jacobson, A. J., Yuan, Z. \& Chen, C. Impedance studies of dense polycrystalline thin films of La $2 \mathrm{NiO} 4+\delta$. Journal of Materials Chemistry 17, 13161320 (2007).

187 Seymour, I. et al. Anisotropic oxygen diffusion in PrBaCo2O5. 5 double perovskites. Solid State Ionics 216, 41-43 (2012).

188 Fierro, J., Tascon, J. \& Tejuca, L. G. Physicochemical properties of LaMnO3: Reducibility and kinetics of O2 adsorption. Journal of Catalysis 89, 209-216 (1984). 
189 Tascón, J. \& Gonzalez Tejuca, L. Adsorption of oxygen on the Perovskite-type oxide LaCoO 3. Z. Phys. Chem.(Wiesbaden) 121, 79-93 (1980).

190 Takeda, Y., Kanno, R., Noda, M., Tomida, Y. \& Yamamoto, O. Cathodic polarization phenomena of perovskite oxide electrodes with stabilized zirconia. Journal of the Electrochemical Society 134, 2656 (1987).

191 Mizusaki, J., Yamauchi, S., Fueki, K. \& Ishikawa, A. Nonstoichiometry of the perovskite-type oxide La1-xSrxCrO3- $\delta$. Solid State Ionics 12, 119-124 (1984).

192 Finklea, H., Chen, X., Gerdes, K., Pakalapati, S. \& Celik, I. Analysis of SOFCs using reference electrodes. Journal of The Electrochemical Society 160, F1055 (2013).

193 Yang, T. et al. An efficient approach for prediction of Warburg-type resistance under working currents. International Journal of Hydrogen Energy 43, 15445-15456 (2018).

194 Finklea, H. O. et al. Counter Electrodes for Electrochemical Evaluation of LSM Electrodes under Polarization. ECS Transactions 78, 677 (2017).

195 Escobar, J. A., Pakalapati, S., Celik, I. B. \& Finklea, H. A correction for impedance measurements using the three point electrode technique in SOFCs. ECS Transactions 25, 391 (2009).

196 Fukunaga, H., Koyama, M., Takahashi, N., Wen, C. \& Yamada, K. Reaction model of dense Sm0. 5Sr0. 5CoO3 as SOFC cathode. Solid State Ionics 132, 279-285 (2000).

197 Chavez, E., Mueller, M., Mogni, L. \& Caneiro, A. Study ofLnBaCo2O6- $\delta(\operatorname{Ln}=\operatorname{Pr}, N d$, Sm and Gd) double perovskites as new cathode material for IT-SOFC. Journal of Physics: Conference Series 167, 012043, doi:10.1088/1742-6596/167/1/012043 (2009).

198 Rautama, E.-L. et al. New member of the "112" family, LaBaCo2O5. 5: synthesis, structure, and magnetism. Chemistry of Materials 21, 102-109 (2009).

199 Caignaert, V., Millange, F., Domenges, B., Raveau, B. \& Suard, E. A new ordered oxygen-deficient manganite perovskite: LaBaMn2O5. 5. Crystal and magnetic structure. Chemistry of materials 11, 930-938 (1999).

200 Fauth, F., Suard, E., Caignaert, V. \& Mirebeau, I. Spin-state ordered clusters in the perovskite NdBaCo 2 O 5.47. Physical Review B 66, 184421 (2002).

201 Subardi, A. \& Fu, Y.-P. in MATEC Web of Conferences. 01026 (EDP Sciences).

202 Klyndyuk, A. Layered Perovskite-Like Oxides 0112 Type. Structure, Properties, and Possible Applications. ChemInform 42, no (2011). 
203 Chernenkov, Y. P. et al. X-ray and neutron diffraction studies of coupled structural phase transitions in DyBaCo2O5. 5. Physics Letters A 365, 166-170 (2007).

204 Zhou, W., Lin, C. T. \& Liang, W. Y. Synthesis and structural studies of the perovskiterelated compound YBaCo2O5+ x. Advanced Materials 5, 735-738 (1993).

205 Mizusaki, J., Mima, Y., Yamauchi, S., Fueki, K. \& Tagawa, H. Nonstoichiometry of the perovskite-type oxides La1- xSrxCoO3- $\delta$. Journal of Solid State Chemistry 80, 102-111 (1989).

206 Mizusaki, J., Tagawa, H., Naraya, K. \& Sasamoto, T. Nonstoichiometry and thermochemical stability of the perovskite-type La1- xSrxMnO3- $\delta$. Solid State Ionics 49, 111-118 (1991).

207 Lankhorst, M. \& Bouwmeester, H. J. Determination of Oxygen Nonstoichiometry and Diffusivity in Mixed Conducting Oxides by Oxygen Coulometric Titration: II. Oxygen Nonstoichiometry and Defect Model for. Journal of the Electrochemical Society 144, 1268 (1997).

208 Nakamura, T., Yashiro, K., Sato, K. \& Mizusaki, J. Oxygen nonstoichiometry and defect equilibrium in La2- xSrxNiO4+ $\delta$. Solid State Ionics 180, 368-376 (2009).

209 Chen, D., Ran, R. \& Shao, Z. Effect of firing temperature on the microstructure and performance of $\mathrm{PrBaCo} 2 \mathrm{O} 5+\delta$ cathodes on $\mathrm{Sm} 0.2 \mathrm{Ce} 0.8 \mathrm{O} 1.9$ electrolytes fabricated by spray deposition-firing processes. Journal of Power Sources 195, 4667-4675, doi:10.1016/j.jpowsour.2010.01.082 (2010).

210 Anjum, U., Vashishtha, S., Sinha, N. \& Haider, M. A. Role of oxygen anion diffusion in improved electrochemical performance of layered perovskite LnBa1-ySryCo2-xFexO5+ $\delta(\mathrm{Ln}=\mathrm{Pr}, \mathrm{Nd}, \mathrm{Gd})$ electrodes. Solid State Ionics 280, 24-29, doi:10.1016/j.ssi.2015.08.002 (2015).

211 Chen, C., Chen, D. \& Ciucci, F. A molecular dynamics study of oxygen ion diffusion in A-site ordered perovskite $\mathrm{PrBaCo}(2) \mathrm{O}(5.5)$ : data mining the oxygen trajectories. Phys Chem Chem Phys 17, 7831-7837, doi:10.1039/c4cp05847j (2015).

212 Li, Y., Gerdes, K. \& Liu, X. Oxygen transport kinetics in infiltrated SOFCs cathode by electrical conductivity relaxation technique. Journal of the Electrochemical Society 160, F554 (2013). 
213 Song, C.-R. \& Yoo, H.-I. Chemical diffusivity of BaTiO3- $\delta$ : I. Experimental determination. Solid State Ionics 120, 141-153 (1999).

214 Jiang, S. \& Wang, W. Novel structured mixed ionic and electronic conducting cathodes of solid oxide fuel cells. Solid State Ionics 176, 1351-1357 (2005).

215 Tarancón, A. et al. Stability, chemical compatibility and electrochemical performance of $\mathrm{GdBaCo} 2 \mathrm{O} 5+\mathrm{x}$ layered perovskite as a cathode for intermediate temperature solid oxide fuel cells. Solid State Ionics 179, 2372-2378 (2008).

216 Pang, S. et al. Characterization of cation-ordered perovskite oxide $\mathrm{LaBaCo} 2 \mathrm{O} 5+\delta$ as cathode of intermediate-temperature solid oxide fuel cells. international journal of hydrogen energy 37, 6836-6843 (2012).

217 Mayeshiba, T. T. \& Morgan, D. D. Factors controlling oxygen migration barriers in perovskites. Solid State Ionics 296, $71-77$ (2016).

218 Xiong, Y. et al. Deposition of platinum particles at LSM/ScSZ/air three-phase boundaries using a platinum current collector. Electrochemical and Solid State Letters 12, B31 (2009).

219 Guan, B., Li, W., Zhang, H. \& Liu, X. Oxygen reduction reaction kinetics in Sr-doped $\mathrm{La} 2 \mathrm{NiO} 4+\delta$ Ruddlesden-Popper phase as cathode for solid oxide fuel cells. Journal of The Electrochemical Society 162, F707 (2015).

220 Pelosato, R., Cordaro, G., Stucchi, D., Cristiani, C. \& Dotelli, G. Cobalt based layered perovskites as cathode material for intermediate temperature Solid Oxide Fuel Cells: A brief review. Journal of Power Sources 298, 46-67 (2015). 